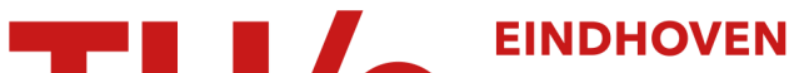 \\ UNIVERSITY OF \\ TECHNOLOGY
}

\section{EDP-convergence for nonlinear fast-slow reaction systems with detailed balance}

\section{Citation for published version (APA):}

Mielke, A., Peletier, M. A., \& Stephan, A. (2021). EDP-convergence for nonlinear fast-slow reaction systems with detailed balance. Nonlinearity, 34(8), 5762-5798. https://doi.org/10.1088/1361-6544/ac0a8a

DOI:

10.1088/1361-6544/ac0a8a

Document status and date:

Published: 01/08/2021

\section{Document Version:}

Publisher's PDF, also known as Version of Record (includes final page, issue and volume numbers)

\section{Please check the document version of this publication:}

- A submitted manuscript is the version of the article upon submission and before peer-review. There can be important differences between the submitted version and the official published version of record. People interested in the research are advised to contact the author for the final version of the publication, or visit the $\mathrm{DOI}$ to the publisher's website.

- The final author version and the galley proof are versions of the publication after peer review.

- The final published version features the final layout of the paper including the volume, issue and page numbers.

Link to publication

\section{General rights}

Copyright and moral rights for the publications made accessible in the public portal are retained by the authors and/or other copyright owners and it is a condition of accessing publications that users recognise and abide by the legal requirements associated with these rights.

- Users may download and print one copy of any publication from the public portal for the purpose of private study or research.

- You may not further distribute the material or use it for any profit-making activity or commercial gain

- You may freely distribute the URL identifying the publication in the public portal.

If the publication is distributed under the terms of Article $25 \mathrm{fa}$ of the Dutch Copyright Act, indicated by the "Taverne" license above, please follow below link for the End User Agreement:

www.tue.nl/taverne

Take down policy

If you believe that this document breaches copyright please contact us at:

openaccess@tue.nl

providing details and we will investigate your claim. 
PAPER • OPEN ACCESS

EDP-convergence for nonlinear fast-slow reaction systems with detailed balance

To cite this article: Alexander Mielke et al 2021 Nonlinearity 345762

View the article online for updates and enhancements. 


\title{
EDP-convergence for nonlinear fast-slow reaction systems with detailed balance*
}

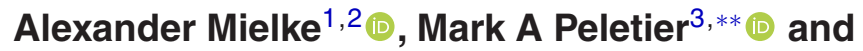 \\ Artur Stephan ${ }^{1}$ \\ 1 Weierstraß-Institut für Angewandte Analysis und Stochastik, Mohrenstraße 39, \\ 10117 Berlin, Germany \\ ${ }^{2}$ Institut für Mathematik, Humboldt-Universität zu Berlin, Unter den Linden 6, \\ 10099 Berlin, Germany \\ 3 Department of Mathematics and Computer Science, Institute for Complex \\ Molecular Systems, TU Eindhoven, 5600 MB Eindhoven, The Netherlands
}

E-mail: alexander.mielke@wias-berlin.de, M.A.Peletier@tue.nl and artur.stephan@wias-berlin.de

Received 16 October 2020, revised 13 April 2021

Accepted for publication 11 June 2021

Published 13 July 2021

\begin{abstract}
We consider nonlinear reaction systems satisfying mass-action kinetics with slow and fast reactions. It is known that the fast-reaction-rate limit can be described by an ODE with Lagrange multipliers and a set of nonlinear constraints that ask the fast reactions to be in equilibrium. Our aim is to study the limiting gradient structure which is available if the reaction system satisfies the detailed-balance condition. The gradient structure on the set of concentration vectors is given in terms of the relative Boltzmann entropy and a cosh-type dissipation potential. We show that a limiting or effective gradient structure can be rigorously derived via EDP-convergence, i.e. convergence in the sense of the energy-dissipation principle for gradient flows. In general, the effective entropy will no longer be of Boltzmann type and the reactions will no longer satisfy mass-action kinetics.

Keywords: reaction system, mass-action kinetics, gradient structure, evolutionary gamma convergence
\end{abstract}

\footnotetext{
* Research partially supported by NWO grant 613.001.552, 'Large Deviations and Gradient Flows' and DFG via SFB 1114 (Project No. 235221301, subproject C05).

${ }^{* *}$ Author to whom any correspondence should be addressed.

Recommended by Dr Konstantin M Khanin.

(c) (i) Original content from this work may be used under the terms of the Creative Commons Attribution 3.0 licence. Any further distribution of this work must maintain attribution to the author(s) and the title of the work, journal citation and DOI.
} 
Mathematics Subject Classification numbers: 49S05, 47J30, 92E20, $34 \mathrm{E} 13$.

(Some figures may appear in colour only in the online journal)

\section{Introduction}

The study of nonlinear reaction systems with different time scales has attracted much attention over the last decades, see e.g. [Bot03, KaK13, WiS17, DLZ18, MiS19, 2020] and the references therein. In this work we consider the simplest case of fast-slow reaction systems with massaction kinetics that have only two time scales, namely 1 and $\varepsilon$,

$$
\dot{c}=\boldsymbol{R}_{\mathrm{sl}}(c)+\frac{1}{\varepsilon} \boldsymbol{R}_{\mathrm{fa}}(c),
$$

where $c \in C:=\left[0, \infty\left[{ }^{i *}\right.\right.$ denotes the vector of the concentrations $c_{i}$ of the $i$ th species $X_{i}$. The typical aim of the above-mentioned work is to derive the limiting equation for the evolution of $c$ on the slow time scale, while the fast reactions are in equilibrium. Under suitable assumptions the limiting equation can be formulated in three equivalent ways:

constrained dynamics:

$$
\dot{c}(t)=\boldsymbol{R}_{\mathrm{sl}}(c(t))+\lambda(t), \quad \lambda(t) \in \Gamma_{\mathrm{fa}} \subset \mathbb{R}^{i_{*}}, \quad \boldsymbol{R}_{\mathrm{fa}}(c(t))=0,
$$

projected dynamics:

$$
\dot{c}(t)=(I-\mathbb{P}(c(t))) \boldsymbol{R}_{\mathrm{sl}}(c(t)), \quad \boldsymbol{R}_{\mathrm{fa}}(c(0))=0,
$$

reduced dynamics:

$$
\dot{\mathrm{q}}(t)=Q_{\mathrm{fa}} \boldsymbol{R}_{\mathrm{sl}}(\Psi(\mathrm{q}(t))), \quad c(t)=\Psi(\mathrm{q}(t)) .
$$

We refer to section 4 for a discussion of these formulations.

The goal of this work is to revisit the same limit process, but now from the point of view of variational evolution. Our starting point is that certain reaction-rate equations (RREs) such as (1.1) can be written as a gradient-flow equation. For a given evolution equation $\dot{u}=\boldsymbol{V}(u)$ on the state space $\mathbf{X}$ we say that it has a gradient structure, if there exists an energy functional $\mathcal{E}: \mathbf{X} \rightarrow \mathbb{R}$ and a (dual) dissipation potential $\mathcal{R}^{*}: T^{*} \mathbf{X} \rightarrow[0, \infty$ [ such that $V(u)=\partial_{\xi} \mathcal{R}^{*}(u,-\mathrm{D} \mathcal{E}(u))$ for all $u \in \mathbf{X}$. This means that the vector field $\boldsymbol{V}$ is generated by two scalar-valued functions $\mathcal{E}$ and $\mathcal{R}$, which are typically motivated by thermodynamical considerations. If $\mathcal{R}^{*}(u, \cdot)$ is quadratic, viz $\mathcal{R}^{*}(u, \xi)=\frac{1}{2}\langle\xi, \mathbb{K}(u) \xi\rangle$, and $\mathbb{K}(u)$ is invertible, then we have a classical gradient system, where $\nabla \mathcal{E}(u):=\mathbb{K}(u) \mathrm{DE}(u)$ is the gradient, and the quadratic form $v \mapsto\left\langle\mathbb{K}(u)^{-1} v, v\right\rangle$ defines a metric tensor.

More general, dissipation potentials are defined via the property that each $\mathcal{R}^{*}(u, \cdot)$ is convex and non-negative with $\mathcal{R}^{*}(u, 0)=0$; a corresponding triple $\left(\mathbf{X}, \mathcal{E}, \mathcal{R}^{*}\right)$ is called a generalized gradient system. Each such system generates a unique gradient-flow equation $\dot{u}=V(u)=$ $\partial_{\xi} \mathcal{R}^{*}(u,-\mathrm{D} \mathcal{E}(u))$. However, for a given evolution equation $\dot{u}=\boldsymbol{V}(u)$ there may be zero, one, or several gradient structures. By the properties of the dissipation potential $\mathcal{R}^{*}$, see section 2.2 , the function $\mathcal{E}$ is a Liapunov function decaying along solutions.

There is no general theory concerning the question when a given RRE has a gradient structure. However, there exists a class of reaction systems that have a natural gradient structure: these are reaction systems with mass-action kinetics where the reactions occur in pairs of forward-backward reactions satisfying the detailed-balance condition (1.3) below. This observation was highlighted in [Mie11, section 3.1] but was observed and used implicitly earlier in 
[ÖtG97, equations $(103)+(113)]$ and [Yon08, section VII]. A different gradient structure already occurs in [Grm10, equation (69)] and has its origin in the thermodynamic considerations in [Mar15] from 1915. The latter structure, which we will call the cosh-type gradient structure as in [MiS19], was mathematically derived in [MPR14, MP*17] from microscopic chemical master equations via a large-deviation principle.

To be specific, we assume that the species $X_{i}, i \in I:=\left\{1, \ldots, i_{*}\right\}$ undergo $r_{*}$ forward-backward reactions according to the mass-action kinetics

$$
\alpha_{1}^{r} X_{1}+\cdots+\alpha_{i_{*}}^{r} X_{i_{*}} \rightleftharpoons \beta_{1}^{r} X_{1}+\cdots+\beta_{i_{*}}^{r} X_{i_{*}},
$$

where $\alpha^{r}=\left(\alpha_{i}^{r}\right)_{i \in I}$ and $\beta^{r}=\left(\beta_{i}^{r}\right)_{i \in I}$ are the stoichiometric vectors in $\mathbb{N}_{0}^{i_{*}}$. The reaction-rate equation (1.1) takes the form

$$
\dot{c}=-\sum_{r=1}^{r_{*}}\left(k_{r}^{\mathrm{fw}} c^{\alpha^{r}}-k_{r}^{\mathrm{bw}} c^{\beta^{r}}\right)\left(\alpha^{r}-\beta^{r}\right), \quad \text { where } c^{\alpha}=c_{1}^{\alpha_{1}} \ldots c_{i_{*}}^{\alpha_{i_{*}}} .
$$

The detailed-balance condition asks for the existence of a positive concentration vector $c_{*}=$ $\left.\left(c_{i}^{*}\right)_{i \in I} \in \mathbf{C}_{+}:=\right] 0, \infty\left[{ }^{i *}\right.$ such that all $r_{*}$ reactions are in

$$
\exists c_{*}=\left(c_{i}^{*}\right)_{i \in I} \in \mathbf{C}_{+} \quad \forall r \in R:=\left\{1, \ldots, r_{*}\right\}: k_{r}^{\mathrm{fw}} c_{*}^{\alpha^{r}}=k_{r}^{\mathrm{bw}} c_{*}^{\beta^{r}} .
$$

This means that there is one equilibrium vector $c_{*}$ such that all reaction pairs are in equilibrium simultaneously. The reaction strength of a reaction pair can then be measured by $\widehat{\kappa}_{r}=k_{r}^{\mathrm{fw}} c_{*}^{\alpha^{r}} / \delta_{r}^{*}=k_{r}^{\mathrm{bw}} c_{*}^{\beta^{r}} / \delta_{r}^{*}$ where $\delta_{r}^{*}=\left(c_{*}^{\alpha^{r}} c_{*}^{\beta^{r}}\right)^{1 / 2}$.

The set of reaction pairs $R$ will be decomposed into slow and fast reactions, namely $R=$ $R_{\mathrm{sl}} \dot{\cup} R_{\mathrm{fa}}$ and by assuming $\widehat{\kappa}_{r}=\kappa_{r}$ for $r \in R_{\mathrm{sl}}$ and $\widehat{\kappa}_{r}=\kappa_{r} / \varepsilon$ for $r \in R_{\mathrm{fa}}$, where $\kappa_{r}$ are fixed numbers. Hence, slow reactions occur on the time scale $O(1)$, whereas fast reactions occur on the time scale $O(\varepsilon)$. The fast-slow RRE now reads

$$
\dot{c}=\boldsymbol{R}_{\mathrm{sl}}(c)+\frac{1}{\varepsilon} \boldsymbol{R}_{\mathrm{fa}}(c) \quad \text { with } \quad \boldsymbol{R}_{x y}(c):=-\sum_{r \in R_{x y}} \kappa_{r} \delta_{r}^{*}\left(\frac{c^{\alpha^{r}}}{c_{*}^{\alpha_{r}}}-\frac{c^{\beta^{r}}}{c_{*}^{\beta_{r}}}\right)\left(\alpha^{r}-\beta^{r}\right) .
$$

Throughout this work, we assume that the equilibrium vector $c_{*}$ does not depend on $\varepsilon$.

The cosh-type gradient structure is now defined in terms of a gradient system $\left(\mathbf{C}, \mathcal{E}, \mathcal{R}_{\varepsilon}^{*}\right)$, where the energy functional is given in terms of the relative Boltzmann entropy

$$
\mathcal{E}(c)=\sum_{i \in I} c_{i}^{*} \lambda_{\mathrm{B}}\left(c_{i} / c_{i}^{*}\right), \quad \text { where } \lambda_{\mathrm{B}}(\rho):=\rho \log \rho-\rho+1,
$$

and the dual dissipation potential $\mathcal{R}_{\varepsilon}^{*}$ in the form

$$
\begin{aligned}
\mathcal{R}_{\varepsilon}^{*}(c, \xi) & =\mathcal{R}_{\mathrm{sl}}^{*}(c, \xi)+\frac{1}{\varepsilon} \mathcal{R}_{\mathrm{fa}}^{*}(c, \xi) \quad \text { with } \\
\mathcal{R}_{x y}^{*}(c, \xi) & =\sum_{r \in R_{x y}} \kappa_{r}\left(c^{\alpha^{r}} c^{\beta^{r}}\right)^{1 / 2} \mathrm{C}^{*}\left(\left(\alpha^{r}-\beta^{r}\right) \cdot \xi\right),
\end{aligned}
$$

where $\mathrm{C}^{*}(\zeta)=4 \cosh (\zeta / 2)-4$ involves the 'cosh structure'. There is now a special and absolutely non-trivial interaction between the Boltzmann entropy, the mass-action law, and the cosh-type gradient structure, which relies on the fact that $\mathrm{DE}(c)$ is the vector of logarithms, 
namely $\xi=\mathrm{DE}(c)=\left(\log \left(c_{i} / c_{i}^{*}\right)\right)_{i}$. Multiplying this by the stoichimetric vectors $\alpha^{r}-\beta^{r}$ and using the logarithm rules we obtain

$$
\left(\alpha^{r}-\beta^{r}\right) \cdot \xi=\left(\alpha^{r}-\beta^{r}\right) \cdot \mathrm{D} \mathcal{E}(c)=\log \left(\frac{c^{\alpha^{r}}}{c_{*}^{\alpha^{r}}}\right)-\log \left(\frac{c^{\beta^{r}}}{c_{*}^{\beta^{r}}}\right) .
$$

For evaluating $\partial_{\xi} \mathcal{R}_{x y}^{*}(c, \xi)$ these terms are inserted into $\left(\mathrm{C}^{*}\right)^{\prime}(\zeta)=2 \sinh (\zeta / 2)=\left(\mathrm{e}^{\zeta}\right)^{1 / 2}-$ $\left(\mathrm{e}^{-\zeta}\right)^{1 / 2}$, which leads to a cancellation of the logarithms and the desired monomials appear after exploiting the square roots $\left(c^{\alpha^{r}} c^{\beta^{r}}\right)^{1 / 2}$ in $\mathcal{R}_{x y}^{*}$ and in $\delta_{r}^{*}=\left(c_{*}^{\alpha^{r}} c_{*}^{\beta^{r}}\right)^{1 / 2}$. Thus, the fast-slow reaction-rate equation (1.4) indeed takes the form of the gradient-flow equation

$$
\dot{c}(t)=\partial_{\xi} \mathcal{R}_{\varepsilon}^{*}(c(t),-\mathrm{D} \mathcal{E}(c(t))) .
$$

In fact, there are many other gradient structures for (1.4), see remark 2.6; however the coshtype gradient structure is special in several aspects: (i) it can be derived via large-deviation principles [MPR14, MP*17], (ii) the dual dissipation potential $\mathcal{R}_{\varepsilon}^{*}$ is independent of $c_{*}$, and (iii) it is stable under general limiting processes, see [ $\mathrm{LM}^{*} 17$, section 3.3]. The property (ii), also called tilt invariance below, will be especially important for us.

The main goal of this paper is to construct the effective gradient system $\left(\mathbf{C}, \mathcal{E}_{\text {eff }}, \mathcal{R}_{\text {eff }}^{*}\right)$ for the given family $\left(\mathbf{C}, \mathcal{E}, \mathcal{R}_{\varepsilon}^{*}\right)$ in the limit $\varepsilon \rightarrow 0^{+}$. Here we use the notion of convergence of gradient system in the sense of the energy-dissipation principle (EDP), shortly called EDPconvergence. This convergence notion was introduced in [DFM19] and further developed in [MMP21, FrL19, MiS19] and is based on the dissipation functionals

$$
\mathfrak{D}_{\varepsilon}^{\eta}(c):=\int_{0}^{T}\left\{\mathcal{R}_{\varepsilon}(c, \dot{c})+\mathcal{R}_{\varepsilon}^{*}(c, \eta-\mathrm{D} \mathcal{E}(c))\right\} \mathrm{d} t,
$$

which are defined for curves $c \in \mathrm{L}^{1}([0, T] ; \mathbf{C})$. Here $\mathcal{R}_{\varepsilon}$ is the primal dissipation potential conjugated to $\mathcal{R}_{\varepsilon}^{*}$, see (2.3). The notion of EDP-convergence with tilting now asks that the two $\Gamma$-convergences $\mathcal{E}_{\varepsilon} \stackrel{\Gamma}{\longrightarrow} \mathcal{E}_{\text {eff }}$ and $\mathfrak{D}_{\varepsilon}^{\eta} \stackrel{\Gamma}{\longrightarrow} \mathfrak{D}_{0}^{\eta}$ (in suitable topologies) and that for all $\eta$ the limit $\mathfrak{D}_{0}^{\eta}$ has the form $\mathfrak{D}_{0}^{\eta}(c)=\int_{0}^{T}\left\{\mathcal{R}_{\text {eff }}(c, \dot{c})+\mathcal{R}_{\text {eff }}^{*}(c, \eta-\mathrm{D} \mathcal{E}(c))\right\} \mathrm{d} t$; see section 3.1 for the exact definitions of $\Gamma$-convergence and EDP-convergence.

Our main result is theorem 3.5, which asserts EDP-convergence with tilting and leading to the effective gradient system $\left(\mathbf{C}, \mathcal{E}_{\text {eff }}, \mathcal{R}_{\text {eff }}\right)$ with

$$
\mathcal{E}_{\text {eff }}=\mathcal{E} \quad \text { and } \quad \mathcal{R}_{\text {eff }}^{*}(c, \xi)=\mathcal{R}_{\mathrm{sl}}^{*}(c, \xi)+\chi_{\Gamma_{\mathrm{fa}}^{\perp}}(\xi),
$$

where $\Gamma_{\mathrm{fa}}=\operatorname{span}\left\{\alpha^{r}-\beta^{r} \mid r \in R_{\mathrm{fa}}\right\}, \Gamma_{\mathrm{fa}}^{\perp}:=\left\{\xi \in \mathbb{R}^{i_{*}} \mid \forall \gamma \in \Gamma_{\mathrm{fa}}: \gamma \cdot \xi=0\right\}$ and $\chi_{A}$ is the characteristic function of convex analysis taking 0 on $A$ and $\infty$ otherwise. The proof relies on three important observations:

(1) Tilting of the relative Boltzmann entropy $\mathcal{E}$ by $\eta$, i.e. replacing $\mathcal{E}(c)$ by $\mathcal{E}(c)-\eta \cdot c$, is equivalent to changing the underlying equilibrium $c_{*}$ to $c_{*}^{\eta}:=\left(\mathrm{e}^{\eta_{i}} c_{i}^{*}\right)_{i \in I}$ (see (3.7)), and $\mathcal{R}_{\varepsilon}^{*}$ is independent of $c_{*}^{\eta}$.

(2) The factor $1 / \varepsilon$ in front of the fast reaction and in front of $\mathcal{R}_{\mathrm{fa}}^{*}$ allows for fast changes of $c$ in the corresponding directions. These directions are given by the fast stoichiometric subspace $\Gamma_{\mathrm{fa}}$. Defining an operator $Q_{\mathrm{fa}}: \mathbb{R}^{i_{*}} \rightarrow \mathbb{R}^{m_{\mathrm{fa}}}$ such that $\operatorname{ker} Q_{\mathrm{fa}}=\Gamma_{\mathrm{fa}}$ and im $Q_{\mathrm{fa}}^{\top}=\Gamma_{\mathrm{fa}}^{\perp}$, a dissipation bound $\mathfrak{D}_{\varepsilon}^{\eta}\left(c^{\varepsilon}\right) \leqslant M_{\text {diss }}<\infty$ does provide a uniform bound on $Q_{\mathrm{fa}} c^{\varepsilon}$ in $W^{1,1}\left([0, T] ; \mathbb{R}^{m_{\mathrm{fa}}}\right)$, but not on $c^{\varepsilon}$ as a whole. The reason is that the blow-up of the dual dissipation potential $\mathcal{R}_{\varepsilon}^{*}(c, \cdot)$ 
along $\Gamma_{\text {fa }}^{\perp}$ is mirrored by a degeneration of the primal dissipation potential $\mathcal{R}_{\varepsilon}$ along $\Gamma_{\text {fa }}$, i.e. for all $v \in \Gamma_{\text {fa }}$ we have $\mathcal{R}_{\varepsilon}(c, v) \rightarrow 0$ for $\varepsilon \rightarrow 0$.

(3) Since $\mathcal{R}_{\varepsilon} \geqslant 0$, a dissipation bound $\mathfrak{D}_{\varepsilon}\left(c^{\varepsilon}\right) \leqslant M_{\text {diss }}$ trivially implies the bound $\int_{0}^{T} \frac{1}{\varepsilon} \mathcal{R}_{\mathrm{fa}}^{*}\left(c^{\varepsilon},-\mathrm{D} \mathcal{E}\left(c^{\varepsilon}\right)\right) \mathrm{d} t \leqslant M_{\mathrm{diss}}$. Analyzing the function $c \mapsto \mathcal{R}_{\mathrm{fa}}(c,-\mathrm{D} \mathcal{E}(c)) \geqslant 0$ for our mass-action reaction kinetics shows that its zero-set is exactly given by the set of equilibria of the fast equation, namely $\mathscr{E}_{\mathrm{fa}}:=\left\{c \in \mathbf{C} \mid \boldsymbol{R}_{\mathrm{fa}}(c)=0\right\}$. Hence, a dissipation bound $\mathfrak{D}_{\varepsilon}\left(c^{\varepsilon}\right) \leqslant M_{\text {diss }}<\infty$ forces the family $\left(c^{\varepsilon}\right)_{\varepsilon}$ to converge towards $\mathscr{E}_{\text {fa }}$, i.e. in the limit the fast reactions have to be in equilibrium for almost all times.

Our analysis is based on the important assumption that the fast reaction system $c^{\prime}(\tau)=$ $\boldsymbol{R}_{\mathrm{fa}}(c(\tau))$ has a unique equilibrium in each flow-invariant subset $\mathbf{C}_{\mathrm{q}}^{\mathrm{fa}}:=\left\{c \in \mathbf{C} \mid Q_{\mathrm{fa}} c=\mathrm{q}\right\}$. This equilibrium is obtained as minimizer of $\mathcal{E}$ on $\mathbf{C}_{\mathrm{q}}^{\mathrm{fa}}$ and is denoted by $\Psi(\mathrm{q})$. Thus, the unique fast-equilibrium condition (UFEC) reads

$$
\mathscr{M}_{\mathrm{sl}}:=\left\{\Psi(\mathrm{q}) \mid \mathrm{q} \in Q_{\mathrm{fa}} \mathbf{C}\right\} \stackrel{! !}{=} \mathscr{E}_{\mathrm{fa}}:=\left\{c \in \mathbf{C} \mid \boldsymbol{R}_{\mathrm{fa}}(c)=0\right\},
$$

which means that there are no 'additional boundary equilibria', see assumption 3.3.

The main difficulty is to show that the information in points (2) and (3) is enough to obtain the compactness necessary for deriving liminf estimate for the $\Gamma$-convergence $\mathfrak{D}_{\varepsilon} \stackrel{\Gamma}{\rightarrow} \mathfrak{D}_{0}$ for the non-convex functionals $\mathfrak{D}_{\varepsilon}$. On the local level, one sees that (2) provides partial control of the temporal oscillations of $\dot{c}^{\varepsilon}$ via the bound on $Q_{\mathrm{fa}} \dot{c}^{\varepsilon}$ in $\mathrm{L}^{1}\left([0, T] ; \mathbb{R}^{m_{\mathrm{fa}}}\right)$, whereas (3) provides strong convergence towards the nonlinear manifold $\mathscr{M}_{\mathrm{sl}}$, which is locally defined via $\mathrm{D} \mathcal{E}(c) \in \Gamma_{\text {fa }}^{\perp}$ (see lemma 3.7). In summary, we are able to show that $\mathfrak{D}_{\varepsilon}\left(c^{\varepsilon}\right) \leqslant M_{\text {diss }}<\infty$ implies that there exists a subsequence such that $c^{\varepsilon_{n}} \rightarrow \widetilde{c}$ in $\mathrm{L}^{1}([0, T] ; \mathbf{C})$ and $Q_{\mathrm{fa}} c^{\varepsilon_{n}} \rightarrow \mathrm{q}$ uniformly in $C^{0}\left([0, T] ; \mathbb{R}^{m_{\mathrm{fa}}}\right)$, where $\widetilde{c}(t)=\Psi(\mathrm{q}(t))$ with $\mathrm{q} \in W^{1,1}\left([0, T] ; \mathbb{R}^{m_{\mathrm{fa}}}\right)$.

As a corollary we obtain that the limiting evolution lies in $\mathscr{M}_{\mathrm{sl}}$ and is governed by the reduced (or coarse grained) equation $\dot{\mathrm{q}}=Q_{\mathrm{fa}} \boldsymbol{R}_{\mathrm{sl}}(\Psi(\mathrm{q})$ ) described by the slow variables $\mathrm{q} \in Q_{\mathrm{fa}} \mathbf{C}$ and a natural gradient structure $\left(Q_{\mathrm{fa}} \mathbf{C}, \mathrm{E}, \mathrm{R}\right)$. Even on the level of the limiting equations our result goes beyond those in [Bot03, DLZ18], since we do not assume that solutions are strictly positive or that the stoichiometric vectors $\gamma^{r}=\alpha^{r}-\beta^{r}, r \in R_{\mathrm{fa}}$, are linearly independent.

For illustration, we close the introduction by a simple example involving $i_{*}=5$ species and one fast and one slow reaction (see section 4.3 for the details under slightly more general conditions):

$$
X_{1}+X_{2} \stackrel{\text { fast }}{\rightleftharpoons} X_{3} \text { and } X_{3}+X_{4} \stackrel{\text { slow }}{\rightleftharpoons} X_{5},
$$

which gives rise to the stoichiometric vectors $\gamma^{\mathrm{fa}}=(1,1,-1,0,0)^{\top}$ and $\gamma^{\mathrm{sl}}=(0,0,1,1,-1)^{\top}$. Assuming the detailed-balance condition with respect to the steady state $c_{*}=(1,1,1,1,1)^{\top}$, the RRE takes the form

$$
\dot{c}=-\frac{\kappa^{\mathrm{fa}}}{\varepsilon}\left(c_{1} c_{2}-c_{3}\right) \gamma^{\mathrm{fa}}-\kappa^{\mathrm{sl}}\left(c_{3} c_{4}-c_{5}\right) \gamma^{\mathrm{sl}}
$$

The limiting reaction system can be described by the slow variables $q=$ $\left(c_{1}+c_{3}, c_{2}+c_{3}, c_{4}, c_{5}\right)^{\top}$ and reads

$$
\dot{\mathrm{q}}=Q_{\mathrm{fa}} \boldsymbol{R}_{\mathrm{sl}}(\Psi(\mathrm{q}))=-\kappa^{\mathrm{sl}}\left(a\left(q_{1}, q_{2}\right) q_{3}-q_{4}\right) \gamma^{\mathrm{sl}},
$$

where the slow manifold takes the form $\Psi(\mathrm{q})=\left(q_{1}, q_{2}, a\left(q_{1}, q_{2}\right), q_{3}, q_{4}\right)$ and the reduced entropy is $\mathrm{E}(\mathrm{q})=\mathcal{E}(\Psi(\mathrm{q}))$. 


\section{Modeling of reaction systems}

We first introduce the classical notation for reaction systems with reaction kinetics according to the mass-action law. After briefly recalling our notation for gradient systems, we show that based on the condition of detailed balance, the RRE is the gradient-flow equation for a suitable gradient system. Next we introduce our class of fast-slow systems, and finally we present a small, but nontrivial example in $\mathbb{R}^{3}$.

\subsection{Mass action law and stoichiometric subspaces}

We consider $i_{*} \in \mathbb{N}$ species $X_{i}$ reacting with each other by $r_{*} \in \mathbb{N}$ reactions. The set of species is denoted by $I=\left\{1, \ldots, i_{*}\right\}$, the set of reactions by $R=\left\{1, \ldots, r_{*}\right\}$, and the $r_{*}$ chemical reactions are given by

$$
\forall r \in R: \quad \sum_{i=1}^{i_{*}} \alpha_{i}^{r} X_{i} \rightleftharpoons \sum_{i=1}^{i_{*}} \beta_{i}^{r} X_{i},
$$

where the stoichiometric vectors $\alpha^{r}, \beta^{r} \in \mathbb{N}_{0}^{i_{*}}$ contain the stoichiometric coefficients. The concentration $c_{i}$ of species $X_{i}$ is nonnegative, the space of concentrations is denoted by

$$
\mathbf{C}=\left[0, \infty\left[^{i_{*}} \subset \mathbb{R}^{i_{*}},\right.\right.
$$

which is the nonnegative cone of $\mathbb{R}^{i_{*}}$. Moreover, we introduce $\mathbf{C}_{+}:=$int $\left.\mathbf{C}=\right] 0, \infty\left[{ }^{i *}\right.$, the interior of the set of concentrations.

The mass-action law for reaction kinetics assumes that the forward and backward reaction fluxes are proportional to the product of the densities of the species, i.e. $j(c)_{r}=-k_{r}^{\mathrm{fw}} c^{\alpha^{r}}+$ $k_{r}^{\mathrm{bw}} c^{\beta^{r}}$, where for stoichiometric vectors $\delta \in \mathbb{N}_{0}^{i_{+}}$the monomials $c^{\delta}$ are given by $\prod_{i=1}^{i_{*}} c_{i}^{\delta_{i}}$. The $\mathrm{RRE}$ of the concentrations $c \in \mathbf{C}$ takes the form

$$
\dot{c}=\boldsymbol{R}(c)=-\sum_{r=1}^{r_{*}}\left(k_{r}^{\mathrm{fw}} c^{\alpha^{r}}-k_{r}^{\mathrm{bw}} c^{\beta^{r}}\right)\left(\alpha^{r}-\beta^{r}\right),
$$

with given forward and backward reaction rates $k_{r}^{\mathrm{fw}}, k_{r}^{\mathrm{bw}}>0$.

For each of the $r$ reactions we introduce the stoichiometric vector $\gamma^{r}:=\alpha^{r}-\beta^{r} \in \mathbb{Z}^{i_{*}}$. The span of all vectors $\gamma^{r}$ is the stoichiometric subspace $\Gamma \subset \mathbb{R}^{i_{*}}$, i.e. $\Gamma:=\operatorname{span}\left\{\gamma^{r} \mid r \in R\right\}$. We do not assume any properties of the stoichiometric vectors, in particular they are not assumed to be linearly independent.

Conservation directions are vectors $q \in \mathbb{R}^{i_{*}}$ such that $q \in \Gamma^{\perp}$ (also written $q \perp \Gamma$ ), where the annihilator $\Gamma^{\perp}$ is defined as $\Gamma^{\perp}=\left\{q \in \mathbb{R}^{i_{*}} \mid \forall \gamma \in \Gamma: q \cdot \gamma=0\right\}$. By construction we have $\boldsymbol{R}(c) \in \Gamma$, thus for all solutions $t \mapsto c(t)$ of the RRE (2.1), the value of $q \cdot c(t)$ is constant, i.e. $q \cdot c$ is a conserved quantity for (2.1). Fixing a basis $\left\{q_{1}, \ldots, q_{m}\right\}$ of $\Gamma^{\perp}$, we introduce a matrix $Q \in \mathbb{R}^{m \times i_{*}}$ by defining its adjoint $Q^{\top}=\left(q_{1}, \ldots, q_{m}\right)$. By construction, $Q^{\top}: \mathbb{R}^{m} \rightarrow \mathbb{R}^{i_{*}}$ is injective, $Q: \mathbb{R}^{i_{*}} \rightarrow \mathbb{R}^{m}$ is surjective, and ker $Q=\Gamma$. The image of the nonnegative cone C under $Q$ is denoted by $\mathscr{Q}$, i.e. $Q: \mathbf{C} \rightarrow \mathscr{Q} \subset \mathbb{R}^{m}$. Fixing a vector $q \in \mathscr{Q}$, we define the stoichiometric subsets

$$
\mathbf{C}_{q}:=\{c \in \mathbf{C} \mid Q c=q\} .
$$

They provide a decomposition $\mathbf{C}=\bigcup_{q \in \mathscr{Q}} \mathbf{C}_{q}$ into affine sets that are invariant under the flow of the RRE (2.1). 
Notation: in the whole paper we consider all vectors as column vectors. In particular $\mathrm{DE}(c) \in X^{*}$ is also a column vector although it is an element of the dual space and might be understood as a covector.

\subsection{Notations for gradient systems}

Following [Mie11, Mie16], we call a triple $(\mathbf{X}, \mathcal{E}, \mathcal{R})$ a (generalized) gradient system $(\mathrm{GS})$ if

(a) The state space $\mathbf{X}$ is a closed and convex subspace of a Banach space $X$,

(b) $\mathcal{E}: \mathbf{X} \rightarrow \mathbb{R}_{\infty}:=\mathbb{R} \cup\{\infty\}$ is a sufficiently smooth functional (such as a free energy, a relative entropy, or a negative entropy, etc),

(c) $\mathcal{R}: \mathbf{X} \times X \rightarrow \mathbb{R}_{\infty}$ is a dissipation potential, which means that for any $u \in \mathbf{X}$ the functional $\mathcal{R}(u, \cdot): X \rightarrow \mathbb{R}_{\infty}$ is lower semicontinuous, nonnegative and convex, and satisfies $\mathcal{R}(u, 0)=0$.

The dynamics of a GS is given by the associated gradient-flow equation that can be formulated in three different, but equivalent ways: as an equation in $X$, in $\mathbb{R}$, or in $X^{*}$ (the dual Banach space of $X$ ), respectively:

(I) Force balance in $X^{*}$ :

$$
0 \in \partial_{\dot{u}} \mathcal{R}(u, \dot{u})+\mathrm{D} \mathcal{E}(u) \quad \subset X^{*},
$$

(II) Power balance in $\mathbb{R}$ :

$$
\mathcal{R}(u, \dot{u})+\mathcal{R}^{*}(u,-\mathrm{D} \mathcal{E}(u))=-\langle\mathrm{D} \mathcal{E}(u), \dot{u}\rangle \quad \in \mathbb{R},
$$

(III) Rate equation in $X$ :

$$
\dot{u} \in \partial_{\xi} \mathcal{R}^{*}(u,-\mathrm{DE}(u)) \quad \subset X .
$$

Here, $\mathcal{R}^{*}$ is the dual dissipation potential obtained by the Legendre-Fenchel transform

$$
\mathcal{R}^{*}(u, \xi):=\sup _{v \in X}\{\langle\xi, v\rangle-\mathcal{R}(u, v)\} .
$$

In general, the partial derivatives $\partial_{\dot{u}} \mathcal{R}(u, \dot{u})$ and $\partial_{\xi} \mathcal{R}^{*}(u, \xi)$ are the possibly set-valued convex subdifferentials.

For a given evolution equation $\dot{u}=\boldsymbol{V}(u)$ we say that it has a gradient structure if there exists a $\mathrm{GS}(\mathbf{X}, \mathcal{E}, \mathcal{R})$ such that the evolution equation is the gradient-flow equation for this GS, namely $\boldsymbol{V}(u)=\partial_{\xi} \mathcal{R}^{*}(u,-\mathrm{D} \mathcal{E}(u))$. We emphasize that a given evolution equation may have none or many gradient structures; see remark 2.6 for the case of our nonlinear reaction systems.

Integrating the power balance (II) in time over $[0, T]$ and using the chain rule for the timederivative of $t \mapsto \mathcal{E}(u(t))$, we obtain another equivalent formulation of the dynamics of the GS, which is called energy-dissipation-balance:

$$
(\mathrm{EDB}) \quad \mathcal{E}(u(T))+\int_{0}^{T}\left\{\mathcal{R}(u, \dot{u})+\mathcal{R}^{*}(u,-\mathrm{D} \mathcal{E}(u))\right\} \mathrm{d} t=\mathcal{E}(u(0)) .
$$

This gives rise to the dissipation functional

$$
\mathfrak{D}(u):=\int_{0}^{T}\left\{\mathcal{R}(u, \dot{u})+\mathcal{R}^{*}(u,-\mathrm{D} \mathcal{E}(u))\right\} \mathrm{d} t,
$$

which is now defined on trajectories $u:[0, T] \mapsto \mathbf{X}$. 
The following energy-dissipation principle (EDP) states that, under natural technical conditions, solving the EDB (2.4) is equivalent to solving any of the three versions of the gradient-flow equation (2.2).

Theorem 2.1 (Energy-dissipation principle, cf [AGS05, proposition 1.4.1] or [Mie16, theorem 3.2]). Assume that $\mathbf{X}$ is a closed convex subset of $X=\mathbb{R}^{i_{*}}$, that $\mathcal{E} \in C^{1}(\mathbf{X}, \mathbb{R})$, and that the dissipation potential $\mathcal{R}(u, \cdot)$ is superlinear uniformly in $u \in \mathbf{X}$. Then, a function $u \in W^{1,1}\left([0, T] ; \mathbb{R}^{i_{*}}\right)$ is a solution of the gradient-flow equation (2.2) if and only if $u$ solves the $E D B(2.4)$.

\subsection{The detailed balance condition induces gradient structures}

Already in section 2.1, we have assumed that each reaction occurs in both forward and backward directions. Such reaction systems are called weakly reversible. A much stronger assumption is the so-called detailed-balance condition which states that there is a strictly positive state $c_{*}=\left(c_{i}^{*}\right) \in \mathbf{C}_{+}$in which all reactions are in equilibrium, i.e. $j_{r}\left(c_{*}\right)=0$ for all $r$ :

$$
\text { (DBC) } \quad \exists c_{*} \in \mathbf{C}_{+} \forall r \in \mathbb{R}: \quad k_{r}^{\mathrm{fw}} c_{*}^{\alpha^{r}}=k_{r}^{\mathrm{bw}} c_{*}^{\beta^{r}} .
$$

Under this assumption, one can rewrite the RRE (2.1) in the symmetric form

$$
\begin{aligned}
& \dot{c}=\boldsymbol{R}(c)=-\sum_{r=1}^{r_{*}} \widehat{\kappa}_{r} \delta_{r}^{*}\left(\frac{c^{\alpha^{r}}}{c_{*}^{\alpha^{r}}}-\frac{c^{\beta^{r}}}{c_{*}^{\beta^{r}}}\right)\left(\alpha^{r}-\beta^{r}\right) \\
& \text { with } \delta_{r}^{*}=\left(c_{*}^{\alpha^{r}} c_{*}^{\beta^{r}}\right)^{1 / 2} \quad \text { and } \quad \widehat{\kappa}_{r}:=k_{r}^{\mathrm{fw}} c_{*}^{\alpha^{r}} / \delta_{r}^{*}=k_{r}^{\mathrm{bw}} c_{*}^{\beta^{r}} / \delta_{r}^{*} .
\end{aligned}
$$

Subsequently, we will use the notion of a reaction system satisfying the detailed-balance condition, or shortly a detailed-balance reaction system.

Definition 2.2 (Detailed-balance reaction systems (DBRS)). For $i_{*}, r_{*} \in \mathbb{N}$ consider the stoichiometric matrices $A=\left(\alpha_{i}^{r}\right) \in \mathbb{N}_{0}^{i_{*} \times r_{*}}$ and $B=\left(\beta_{i}^{r}\right) \in \mathbb{N}_{0}^{i_{*} \times r_{*}}$ and the vectors $\left.c_{*}=\left(c_{i}^{*}\right) \in\right] 0, \infty\left[{ }^{i *}\right.$ and $\left.\widehat{\kappa}=\left(\widehat{\kappa}_{r}\right) \in\right] 0, \infty\left[^{r_{*}}\right.$. Then, the quadruple $\left(A, B, c_{*}, \widehat{\kappa}\right)$ is called a DBRS with $i_{*}$ species and $r_{*}$ reactions. The associated RRE is given by (2.6).

It was observed in [Mie11] (but see also [ÖtG97, equation $(103)+(113)]$ and [Yon08, section VII] for earlier, but implicit statements) that RREs in this form have a gradient structure. Here we will use the gradient structure derived in [MP* 17$]$ by a large-deviation principle from a microscopic Markov process. In remark 2.6 we will shortly comment on other possible gradient structures.

With $\mathbf{C}$ as above we define the energy as the relative Boltzmann entropy

$$
\begin{aligned}
\mathcal{E}: \begin{cases}\mathbf{C} & \rightarrow \\
c & \mapsto \sum_{i=1}^{i_{*}} c_{i}^{*} \lambda_{\mathrm{B}}\left(c_{i} / c_{i}^{*}\right),\end{cases} \\
\lambda_{\mathrm{B}}(r)= \begin{cases}r \log r-r+1 & \text { for } r>0, \\
1 & \text { for } r=0, \\
\infty & \text { for } r<0 .\end{cases}
\end{aligned}
$$


The dissipation functional $\mathcal{R}$ will be defined by specifying the dual dissipation potential $\mathcal{R}^{*}$ of 'cosh-type' as

$$
\begin{aligned}
& \mathcal{R}^{*}:\left\{\begin{array}{cc}
\mathbf{C} \times \mathbb{R}^{i_{*}} & \rightarrow \\
(c, \xi) & \mapsto \sum_{r=1}^{r_{*}} \widehat{\kappa}_{r} \sqrt{c^{\alpha^{r}} c^{\beta^{r}}} \mathrm{C}^{*}\left(\left(\alpha^{r}-\beta^{r}\right) \cdot \xi\right),
\end{array}\right. \\
& \text { with } \mathrm{C}^{*}(\zeta)=4 \cosh (\zeta / 2)-4 .
\end{aligned}
$$

We will often use the following formulas for $\mathrm{C}^{*}$ :

$$
\begin{aligned}
& \text { (a) } \mathrm{C}^{*}(\log p-\log q)=2 \frac{(\sqrt{p}-\sqrt{q})^{2}}{\sqrt{p q}}, \\
& \begin{array}{ll}
\text { (b) }\left(\mathrm{C}^{*}\right)^{\prime}(\zeta)=\mathrm{e}^{\zeta / 2}-\mathrm{e}^{-\zeta / 2}, \quad \text { (c) }\left(\mathrm{C}^{*}\right)^{\prime}(\log p-\log q)=\frac{p-q}{\sqrt{p q}}
\end{array}
\end{aligned}
$$

The following result is also easily checked by direct calculations using (2.8)(b) and the logarithm rules

$$
\alpha^{r} \mathrm{DE}(c)=\log \left(c^{\alpha^{r}}\right)-\log \left(c_{*}^{\alpha^{r}}\right)=\log \left(c^{\alpha^{r}} / c_{*}^{\alpha^{r}}\right) .
$$

This identity also follows as a special case of remark 2.6. The primal dissipation potential $\mathcal{R}$ is given by the Legendre-Fenchel transformation:

$$
\mathcal{R}(u, v)=\sup \left\{\xi \cdot v-\mathcal{R}^{*}(c, \xi) \mid \xi \in \mathbb{R}^{i_{*}}\right\} .
$$

Proposition 2.3 (Gradient structure, [MP*17, theorem 3.6]). The RRE (2.6) is the gradient-flow equation associated with the cosh-type $G S(\mathbf{C}, \mathcal{E}, \mathcal{R})$ with $\mathcal{E}$ and $\mathcal{R}$ given in (2.7), where $\mathcal{R}$ and $\mathcal{R}^{*}$ are related by Legendre-Fenchel transform, see (2.3).

An important property of this gradient structure, which is not shared with the ones discussed in remark 2.6 below, is that the dissipation potential $\mathcal{R}^{*}$ does not depend on the equilibrium state $c_{*}$, see also section 2.5 for an example. This property might seem to be an artifact of our special choice of the definition of $\widehat{\kappa}_{r}$ in terms of $c_{*}$; however, it is an intrinsic property that will be even more relevant when we use 'tilting' in our main result theorem 3.5, which states the 'EDP-convergence with tilting'. In [MiS19, proposition 4.1] it was shown that this tilt-invariance is a special property of the cosh-gradient structure; see also remark 2.6.

Moreover, we have identified $c_{*}$ as a 'static' property of the RRE (2.6), whereas the stoichiometric matrices $A, B \in \mathbb{N}_{0}^{i_{*} \times r_{*}}$ and the reaction coefficients $\widehat{\kappa}_{r}$ encode the 'dissipative' properties.

Because we are going to use the EDP, we explicitly state the cosh-type dissipation functional given by

$$
\mathfrak{D}(c)=\int_{0}^{T}\left\{\mathcal{R}(c, \dot{c})+\mathcal{R}^{*}(c,-\mathrm{D} \mathcal{E}(c))\right\} \mathrm{d} t=\int_{0}^{T}\{\mathcal{R}(c, \dot{c})+\mathcal{S}(c)\} \mathrm{d} t .
$$

We will mostly write the dissipation functional $\mathfrak{D}$ in the first ' $\mathcal{R}+\mathcal{R}$ ' form' to highlight its duality structure. However, for mathematical purposes it will be advantageous to use the second representation via the slope function

$$
\mathcal{S}(c): \mathbf{C} \rightarrow\left[0, \infty\left[; c \mapsto \mathcal{S}(c):=\sum_{r=1}^{r_{*}} 2 \widehat{\kappa}_{r} \delta_{r}^{*}\left(\left(\frac{c^{\alpha^{r}}}{c_{*}^{\alpha^{r}}}\right)^{1 / 2}-\left(\frac{c^{\beta^{r}}}{c_{*}^{\beta^{r}}}\right)^{1 / 2}\right)^{2},\right.\right.
$$


which is continuous on $\mathbf{C}$ and satisfies $\mathcal{S}(c)=\mathcal{R}^{*}(c,-\mathrm{D} \mathcal{E}(c))$ for $c \in \mathbf{C}_{+}$. Sometimes $\mathcal{S}$ is also called the (discrete) Fisher information as it corresponds to $\int_{\Omega} 4 k|\nabla \sqrt{\rho}|^{2} \mathrm{~d} x=\int_{\Omega} k|\nabla \rho|^{2} / \rho \mathrm{d} x$ in the diffusion case.

A special feature of DBRS is that all equilibria have the property that they provide an equilibrium to each individual reaction $r \in R$, where we do not need linear independence of $\left(\gamma^{r}\right)_{r \in R}$, see also [MHM15, section 2] or [Mie17].

Lemma 2.4 (Equilibria in DBRS). Let $\left(A, B, c_{*}, \widehat{\kappa}\right)$ be a DBRS with slope function $\mathcal{S}$ defined in (2.12). Then, the following identities hold:

$$
\begin{aligned}
\mathscr{E}_{\boldsymbol{R}} & :=\{c \in \mathbf{C} \mid \boldsymbol{R}(c)=0\}=\{c \in \mathbf{C} \mid \mathcal{S}(c)=0\} \\
& =\left\{c \in \mathbf{C} \mid \forall r \in R: \frac{c^{\alpha^{r}}}{c_{*}^{\alpha^{r}}}=\frac{c^{\beta^{r}}}{c_{*}^{\beta^{r}}}\right\} .
\end{aligned}
$$

Moreover, if $\widetilde{c}_{*} \in \mathscr{E}_{\boldsymbol{R}} \cap \mathbf{C}_{+}$, then the two $\operatorname{DBRS}\left(A, B, c_{*}, \widehat{\kappa}\right)$ and $\left(A, B, \widetilde{c}_{*}, \widehat{\kappa}\right)$ generate the same RRE.

Proof. Step 1. For $c \in \mathbf{C}_{+}$the gradient structure $\boldsymbol{R}(c)=\partial \mathcal{R}^{*}(c,-\mathrm{D} \mathcal{E}(c))$ of the DBRS gives

$$
\mathcal{R}(c, \boldsymbol{R}(c))+\mathcal{R}^{*}(c,-\mathrm{D} \mathcal{E}(c))=-\mathrm{D} \mathcal{E}(c) \cdot \boldsymbol{R}(c) .
$$

Thus, $\boldsymbol{R}(c)=0$ implies $\mathcal{S}(c)=\mathcal{R}^{*}(c,-\mathrm{DE}(c))=0$, and since $\mathcal{S}(c)$ is the sum of $r_{*}$ nonnegative terms ( $\operatorname{cf}(2.12))$ we conclude $\frac{c^{\alpha^{r}}}{c_{*}^{\alpha^{r}}}=\frac{c^{\beta^{r}}}{c^{\beta^{r}}}$ as desired.

Step 2. If $c \in \partial \mathbf{C}$ satisfies $\boldsymbol{R}(c)=0$, then consider $c_{\delta}=c+\delta c_{*} \in \mathbf{C}_{+}$for $\delta \in$ ] 0, 1 [. With $\left|\boldsymbol{R}\left(c_{\delta}\right)\right| \leqslant C_{0} \delta, \mathcal{R}\left(c_{\delta}, v\right) \geqslant 0$, and $\left|\mathrm{D} \mathcal{E}\left(c_{\delta}\right)\right| \leqslant i_{*} \log (1 / \delta)$ we find

$$
\begin{aligned}
\mathcal{S}\left(c_{\delta}\right) & =\mathcal{R}^{*}\left(c_{\delta},-\mathrm{D} \mathcal{E}\left(c_{\delta}\right)\right)=-\mathrm{D} \mathcal{E}\left(c_{\delta}\right) \cdot \boldsymbol{R}\left(c_{\delta}\right)-\mathcal{R}\left(c_{\delta}, \boldsymbol{R}\left(c_{\delta}\right)\right) \\
& \leqslant i_{*} C_{0} \delta \log (1 / \delta)+0
\end{aligned}
$$

Using the continuity of $\mathcal{S}$ we obtain $\mathcal{S}(c)=\lim _{\delta \rightarrow 0^{+}} \mathcal{S}\left(c_{\delta}\right)=0$ and conclude as in step 1 .

Step 3. The equilibrium condition of step 1 implies $\widetilde{c}_{*}^{\beta^{r}} / \widetilde{c}_{*}^{\alpha^{r}}=c_{*}^{\beta^{r}} / c_{*}^{\alpha^{r}}=: \mu_{r}^{2}$ for all $r \in$ $R$. Because in the RRE (2.6) only the terms $\delta_{*}^{r} / c_{*}^{\alpha^{r}}=\left(c_{*}^{\beta^{r}} / c_{*}^{\alpha^{r}}\right)^{1 / 2}=\mu_{r}$ and $\delta_{*}^{r} / c_{*}^{\beta^{r}}=1 / \mu_{r}$ appear, the last statement follows.

The next lemma shows that $\mathcal{R}(c, \cdot)$ forbids velocities $v$ outside of the stoichiometric subspace $\Gamma$. Moreover, for all trajectories $c:[0, T] \rightarrow \mathbf{C}$ with $\mathfrak{D}(c)<\infty$, which are much more than the solutions of the RRE (2.1), we find that they have to lie in one stoichiometric subset $\mathbf{C}_{q}$, i.e. the conserved quantities are already encoded in $\mathfrak{D}$.

Below we use the characteristic function $\chi_{A}$ of convex analysis, which is defined via $\chi_{A}(v)=0$ for $v \in A$ and $\chi_{A}(v)=\infty$ otherwise.

Lemma 2.5 (Conserved quantities via $\mathfrak{D})$. Let $\Gamma, Q, \mathbf{C}_{q}$, and $\mathscr{Q}$ be defined as in section 2.1, and let $\mathcal{R}^{*}$ and $\mathcal{R}$ be defined as in (2.7b) and (2.10), respectively.

(a) For all $(c, v) \in \mathbf{C} \times \mathbb{R}^{i_{*}}$ we have $\mathcal{R}(c, v) \geqslant \chi_{\Gamma}(v)$.

(b) If $c \in W^{1,1}([0, T] ; C)$ satisfies $\mathfrak{D}(c)<\infty$, then $Q \dot{c}=0$ a.e., or equivalently there exists $q \in \mathscr{Q}$ such that $c(t) \in \boldsymbol{C}_{q}$ for all $t \in[0, T]$.

Proof. Using $\gamma^{r}=\alpha^{r}-\beta^{r}$ we find $\mathcal{R}^{*}(c, \xi)=0$ for $\xi \perp \Gamma=\operatorname{ker}(Q)$ and conclude

$$
\mathcal{R}(c, v)=\sup _{\xi}\left(\xi \cdot v-\mathcal{R}^{*}(c, \xi)\right) \geqslant \sup _{\xi \perp \Gamma}\left(\xi \cdot v-\mathcal{R}^{*}(c, \xi)\right)=\sup _{\xi \perp \Gamma}(\xi \cdot v)=\chi_{\Gamma}(v) .
$$


This proves part (a).

The bound $\mathfrak{D}(c)<\infty$ implies that $\int_{0}^{T} \mathcal{R}(c, \dot{c}) \mathrm{d} t<\infty$ and hence $\dot{c} \in \Gamma=\operatorname{ker}(Q)$ a.e. This proves $Q \dot{c}(t)=0$ a.e. and by the absolute continuity of $c$, the function $t \mapsto Q c(t)$ must be constant. Hence part (b) is established as well.

Remark 2.6 (Different gradient structures). We emphasize that the symmetric RRE (2.6), which was obtained from the DBC, indeed has many other gradient structures with the same relative entropy $\mathcal{E}$ given in (2.7a). Choosing arbitrary smooth and strictly convex functions $\Phi_{r}: \mathbb{R} \rightarrow\left[0, \infty\left[\right.\right.$ with $\Phi_{r}(0)=0$ and $\Phi_{r}(-\zeta)=\Phi_{r}(\zeta)$ we may define

$$
\begin{aligned}
\mathcal{R}_{\Phi}^{*}(c, \xi) & =\sum_{r=1}^{r_{*}} \widehat{\kappa}_{r} \delta_{r}^{*} \Lambda_{r}\left(\frac{c^{\alpha^{r}}}{c_{*}^{\alpha^{r}}}, \frac{c^{\beta^{r}}}{c_{*}^{\beta^{r}}}\right) \Phi_{r}\left(\left(\alpha^{r}-\beta^{r}\right) \cdot \xi\right) \\
\text { with } \Lambda_{r}(a, b) & =\frac{a-b}{\Phi_{r}^{\prime}(\log a-\log b)}
\end{aligned}
$$

and $\delta_{r}^{*}=\left(c_{*}^{\alpha^{r}} c_{*}^{\beta^{r}}\right)^{1 / 2}$. Note that $\Lambda_{r}$ can be smoothly extended by $\Lambda_{r}(a, a)=a / \Phi_{r}^{\prime \prime}(0)$.

To show that the GS $\left(\mathbf{C}, \mathcal{E}, \mathcal{R}_{\Phi}\right)$ indeed generates (2.6) as the associated gradient-flow equation, it suffices to consider the $r$ th reaction pair, because the dual potential $\mathcal{R}_{\Phi}^{*}$ is additive in the reaction pairs. Inserting $\mathrm{D} \mathcal{E}(c)=\left(\log \left(c_{i} / c_{i}^{*}\right)\right)_{i=1, \ldots, i_{*}}$ we obtain the relation

$$
\begin{aligned}
\mathrm{D}_{\xi} \mathcal{R}_{\Phi_{r}}^{*}(c,-\mathrm{D} \mathcal{E}(c)) \\
=\widehat{\kappa}_{r} \delta_{r}^{*} \Lambda_{r}\left(\frac{c^{\alpha^{r}}}{c_{*}^{\alpha^{r}}}, \frac{c^{\beta^{r}}}{c_{*}^{\beta^{r}}}\right) \Phi_{r}^{\prime}\left(\left(\alpha^{r}-\beta^{r}\right) \cdot(-\mathrm{D} \mathcal{E}(c))\right)\left(\alpha^{r}-\beta^{r}\right) \\
\stackrel{(2.9)}{=}-\widehat{\kappa}_{r} \delta_{r}^{*} \Lambda_{r}\left(\frac{c^{\alpha^{r}}}{c_{*}^{\alpha^{r}}}, \frac{c^{\beta^{r}}}{c_{*}^{\beta^{r}}}\right) \Phi_{r}^{\prime}\left(\log \left(\frac{c^{\alpha^{r}}}{c_{*}^{\alpha^{r}}}\right)-\log \left(\frac{c^{\beta^{r}}}{c_{*}^{\beta^{r}}}\right)\right) \\
\quad \times\left(\alpha^{r}-\beta^{r}\right)=-\widehat{\kappa}_{r} \delta_{r}^{*}\left(\frac{c^{\alpha^{r}}}{c_{*}^{\alpha^{r}}}-\frac{c^{\beta^{r}}}{c_{*}^{\beta^{r}}}\right)\left(\alpha^{r}-\beta^{r}\right),
\end{aligned}
$$

which is the desired result.

The choice $\Phi_{r}(\zeta)=\zeta^{2} / 2$ was used in [Mie11], while here we use $\Phi_{r}=\mathrm{C}^{*}$ leading to

$$
\Lambda_{r}(a, b)=(a b)^{1 / 2} \quad \text { and } \quad \delta_{r}^{*} \Lambda_{r}\left(\frac{c^{\alpha^{r}}}{c_{*}^{\alpha^{r}}}, \frac{c^{\beta^{r}}}{c_{*}^{\beta^{r}}}\right)=\left(c^{\alpha^{r}} c^{\beta^{r}}\right)^{1 / 2}
$$

This is the desired term in (2.7b) that is independent of $c_{*}$, while for other choice of $\Phi_{r}$ the last term will depend on $c_{*}$ (see [MiS19]).

\subsection{Fast-slow reaction-rate equation}

We assume that some reactions are fast with reaction coefficients $\widehat{\kappa}_{r}^{\varepsilon}=\kappa_{r} / \varepsilon$, while the others are slow with reaction coefficients $\widehat{\kappa}_{r}^{\varepsilon}=\kappa_{r}$ (of order 1 ). Here we assume that the set or reaction indices $R=\left\{1, \ldots, r_{*}\right\}$ decomposes into $R_{\mathrm{sl}} \dot{\cup} R_{\mathrm{fa}}$. For simplicity we assume that the detailedbalance steady state $c_{*}$ is independent of $\varepsilon$, but a soft dependence with a limit $c_{*}^{\varepsilon} \rightarrow c_{*} \in \mathbf{C}_{+}$ 
could be allowed as well.

$$
\begin{aligned}
& \dot{c}=\boldsymbol{R}_{\varepsilon}(c)=-\sum_{r=1}^{r_{*}} \widehat{\kappa}_{r}^{\varepsilon} \delta_{r}^{*}\left(\frac{c^{\alpha^{r}}}{c_{*}^{\alpha^{r}}}-\frac{c^{\beta^{r}}}{c_{*}^{\beta^{r}}}\right)\left(\alpha^{r}-\beta^{r}\right)=\boldsymbol{R}_{\mathrm{sl}}(c)+\frac{1}{\varepsilon} \boldsymbol{R}_{\mathrm{fa}}(c) \\
& \text { with } \boldsymbol{R}_{x y}(c)=\sum_{r \in R_{x y}} \kappa_{r} \delta_{r}^{*}\left(\frac{c^{\alpha^{r}}}{c_{*}^{\alpha^{r}}}-\frac{c^{\beta^{r}}}{c_{*}^{\beta^{r}}}\right)\left(\alpha^{r}-\beta^{r}\right) \quad \text { for } x y \in\{\mathrm{sl}, \mathrm{fa}\} .
\end{aligned}
$$

Obviously, for each $\varepsilon>0$ we have a cosh-type gradient structure $\left(\mathbf{C}, \mathcal{E}, \mathcal{R}_{\varepsilon}\right)$ with

$$
\begin{aligned}
\mathcal{R}_{\varepsilon}^{*}(c, \xi) & =\mathcal{R}_{\mathrm{sl}}^{*}(c, \xi)+\frac{1}{\varepsilon} \mathcal{R}_{\mathrm{fa}}^{*}(c, \xi) \quad \text { with } \\
\mathcal{R}_{x y}^{*}(c, \xi) & =\sum_{r \in R_{x y}} \kappa_{r} \sqrt{c^{\alpha^{r}} c^{\beta^{r}}} \mathrm{C}^{*}\left(\left(\alpha^{r}-\beta^{r}\right) \cdot \xi\right) .
\end{aligned}
$$

The aim of this paper is to investigate the behavior of the gradient structures $\left(\mathbf{C}, \mathcal{E}, \mathcal{R}_{\varepsilon}\right)$ in the limit $\varepsilon \rightarrow 0^{+}$. In particular, we study the $\Gamma$-limit of the induced dissipation functionals $\mathfrak{D}_{\varepsilon}$ obtained as in (2.11) but with the duality pair $\mathcal{R}_{\varepsilon}+\mathcal{R}_{\varepsilon}^{*}$.

At this stage we report on well-known results (see e.g. [Bot03, DLZ18]) about the limit evolution for $\varepsilon \rightarrow 0^{+}$. For small times of order $\varepsilon$ the fast system $\boldsymbol{R}_{\mathrm{fa}}$ will dominate, while for $t \in[\sqrt{\varepsilon}, T]$ a slow dynamics takes place where the slow reactions drive the evolution and the fast reactions remain in equilibrium.

To be more precise we introduce the fast time scale $\tau=t / \varepsilon$ such that in terms of $\tau$ we obtain the rescaled system $c^{\prime}(\tau)=\varepsilon \boldsymbol{R}_{\mathrm{sl}}(c(\tau))+\boldsymbol{R}_{\mathrm{fa}}(c(\tau))$. For $\varepsilon \rightarrow 0^{+}$we obtain the fast system

$$
c^{\prime}(\tau)=\boldsymbol{R}_{\mathrm{fa}}(c(\tau)), \quad c(0)=c_{0} .
$$

This is again an RRE satisfying the detailed-balance condition and all constructions introduced in sections 2.1 and 2.3. In particular we obtain the fast stoichiometric subspace

$$
\Gamma_{\mathrm{fa}}:=\operatorname{span}\left\{\gamma^{r} \in \mathbb{Z}^{i_{*}} \mid r \in R_{\mathrm{fa}}\right\} \subset \Gamma \subset \mathbb{R}^{i_{*}} .
$$

For the annihilator $\Gamma_{\mathrm{fa}}^{\perp}:=\left\{q \in \mathbb{R}^{i_{*}} \mid \forall \gamma \in \Gamma_{\mathrm{fa}}: q \cdot \gamma=0\right\}$ we have $\Gamma^{\perp} \subset \Gamma_{\mathrm{fa}_{\mathrm{a}}}^{\perp}$ and $m_{\mathrm{fa}}:=\operatorname{dim} \Gamma_{\mathrm{fa}}^{\perp} \geqslant m=\operatorname{dim} \Gamma^{\perp}$. Thus, we can extend the basis $\left\{q_{1}, \ldots, q_{m}\right\}$ for $\Gamma^{\perp}$ to a basis $\left\{q_{1}, \ldots, q_{m}, \ldots, q_{m_{\mathrm{fa}}}\right\}$ for $\Gamma_{\mathrm{fa}}^{\perp}$ and define the conservation operator $Q_{\mathrm{fa}}: \mathbb{R}^{i_{*}} \rightarrow \mathbb{R}^{m_{\mathrm{fa}}}$ via

$$
Q_{\mathrm{fa}}^{\top}:=\left(q_{1}, \ldots, q_{m_{\mathrm{fa}}}\right): \mathbb{R}^{m_{\mathrm{fa}}} \rightarrow \mathbb{R}^{i_{*}} \text { and set } \quad \mathbf{Q}:=\left\{Q_{\mathrm{fa}} c \in \mathbb{R}^{m_{\mathrm{fa}}} \mid c \in \mathbf{C}\right\} .
$$

In particular, the important defining relations of $Q_{\mathrm{fa}}$ are

$$
\operatorname{ker} Q_{\mathrm{fa}}=\Gamma_{\mathrm{fa}} \quad \text { and } \quad \operatorname{im} Q_{\mathrm{fa}}^{\top}=\Gamma_{\mathrm{fa}}^{\perp} .
$$

Of course, our interest lies in the case $0 \leqslant m \supsetneqq m_{\mathrm{fa}} \supsetneqq i_{*}$. In that case the mapping $c \mapsto Q c$ yields fewer conserved quantities for the full fast-slow RRE (2.15) than the mapping $c \mapsto q=$ $Q_{\mathrm{fa}} c$ supplies for the fast RRE (2.17). We call $\mathrm{q} \in \mathbf{Q}$ the slow variables, as they may still vary on the slow time scale. In particular, the decomposition of $\mathbf{C}$ into fast stoichiometric subsets

$$
\mathbf{C}=\bigcup_{\mathbf{q} \in \mathbf{Q}} \mathbf{C}_{\mathrm{q}}^{\mathrm{fa}} \quad \text { where } \quad \mathbf{C}_{\mathrm{q}}^{\mathrm{fa}}:=\left\{c \in \mathbf{C} \mid Q_{\mathrm{fa}} c=\mathbf{q}\right\}
$$

is finer than $\mathbf{C}=\bigcup_{q \in \mathcal{Q}} \mathbf{C}_{q}$. 
Starting from a general initial condition $c_{0}$, one can show that the solutions $c^{\varepsilon}:[0, T] \rightarrow \mathbf{C}$ of the fast-slow RRE (2.15) have a limit $c^{0}:[0, T] \rightarrow \mathbf{C}$, but this limit may not be continuous at $t=0$. On the short time scale $\tau=t / \varepsilon$ we may define $\widetilde{c}^{\varepsilon}(\tau)=c^{\varepsilon}(\varepsilon \tau)$ which has a limit $\widetilde{c}^{0}$ : $\left[0, \infty\left[\rightarrow \mathbf{C}\right.\right.$ satisfying the fast RRE (2.17) and having a limit $\bar{c}_{0}:=\lim _{\tau \rightarrow \infty} \widetilde{c}^{0}(\tau)$ with $\boldsymbol{R}_{\mathrm{fa}}\left(\bar{c}_{0}\right)=$ 0 . Hence, we define the set of fast equilibria

$$
\mathscr{E}_{\mathrm{fa}}:=\left\{c \in \mathbf{C} \mid \boldsymbol{R}_{\mathrm{fa}}(c)=0\right\}=\left\{c \in \mathbf{C} \mid \forall r \in R_{\mathrm{fa}}: \frac{c^{\alpha^{r}}}{c_{*}^{\alpha^{r}}}=\frac{c^{\beta^{r}}}{c_{*}^{\beta^{r}}}\right\}
$$

such that for $\tau \in\left[0, \infty\left[\right.\right.$ the solution $\widetilde{c}^{0}(\tau)$ describes the approach to the slow manifold and $\bar{c}_{0} \in \mathscr{E}_{\mathrm{fa}}$. On the time scale of order 1 , the limits $c^{0}(t)$ of the solutions $c^{\varepsilon}(t)$ satisfy $c^{0}(t) \in \mathscr{E}_{\mathrm{fa}}$ for all $t \in[0, T]$, and one has the matching condition $\bar{c}_{0}=\lim _{t \rightarrow 0^{+}} c^{0}(t)$.

The evolution of the solutions $c^{0}$ within $\mathscr{E}_{\mathrm{fa}}$ is driven by the slow reactions only; the fast reactions keep the solution on the fast-equilibrium manifold $\mathscr{E}_{\mathrm{fa}}$. In particular, it can be shown (see [Bot03, DLZ18] or [MiS19] for the linear case) that $c^{0}$ satisfies the limiting equation

$$
\dot{c}(t)=\boldsymbol{R}_{\mathrm{sl}}(c(t))+\lambda(t) \quad \text { with } \quad c(t) \in \mathscr{E}_{\mathrm{fa}} \text { and } \lambda(t) \in \Gamma_{\mathrm{fa}}, \quad c(0)=\bar{c}_{0} .
$$

The result of our paper is quite different: we will pass to the limit in the GSs $\left(\mathbf{C}, \mathcal{E}, \mathcal{R}_{\varepsilon}\right)$ directly and obtain an effective $\mathrm{GS}\left(\mathbf{C}, \mathcal{E}, \mathcal{R}_{\text {eff }}\right)$, see theorem 3.5 . As a consistency check, we will show in section 4 that the gradient-flow equation for $\left(\mathbf{C}, \mathcal{E}, \mathcal{R}_{\text {eff }}\right)$ is indeed identical to the limiting equation (2.21), see proposition 4.4.

\subsection{A simple example for a fast-slow system}

As a guiding example, we consider a reaction system consisting of three species $X_{i}, i=$ $1,2,3=i_{*}$, which interact via $r_{*}=2$ reactions, one being slow and one being fast:

$$
\text { slow: } X_{1} \rightleftharpoons X_{3} \quad \text { fast: } X_{1}+X_{2} \rightleftharpoons 2 X_{3} \text {. }
$$

Hence, the stoichiometric vector are given by

$$
\begin{array}{lll}
\alpha^{1}=(1,0,0)^{\top}, & \beta^{1}=(0,0,1)^{\top}, & \gamma^{1}=(1,0,-1)^{\top}, \\
\alpha^{2}=(1,1,0)^{\top}, & \beta^{2}=(0,0,2)^{\top}, & \gamma^{2}=(1,1,-2)^{\top} .
\end{array}
$$

Hence, one can easily check that the space of conserved quantities is $\operatorname{span}\left((1,1,1)^{\top}\right) \in \mathbb{R}^{3}$ which defines the matrix $Q=(1,1,1) \in \mathbb{R}^{1 \times 3}$.

We have $R=R_{\mathrm{fa}} \cup R_{\mathrm{sl}}=\{1\} \cup\{2\}$ and the RRE reads

$$
\dot{c}=\boldsymbol{R}_{\varepsilon}(c)=\left(c_{3}-3 c_{1}\right)\left(\begin{array}{c}
1 \\
0 \\
-1
\end{array}\right)+\frac{1}{\varepsilon}\left(c_{3}^{2}-c_{1} c_{2}\right)\left(\begin{array}{c}
1 \\
1 \\
-2
\end{array}\right) .
$$

The nontrivial equilibria of this RRE are given by $c_{*}=\left(c_{1}^{*}, c_{2}^{*}, c_{3}^{*}\right)^{\top}=\sigma(1,9,3)^{\top}$ for $\sigma>0$. All these $c_{*}$ satisfy the detailed balance condition, and (2.22) takes the symmetric form (2.6), viz

$$
\begin{aligned}
& \dot{c}=-\kappa_{1} \delta_{1}^{*}\left(\frac{c_{1}}{c_{1}^{*}}-\frac{c_{3}}{c_{3}^{*}}\right)\left(\begin{array}{c}
1 \\
0 \\
-1
\end{array}\right)-\frac{\kappa_{2}}{\varepsilon} \delta_{2}^{*}\left(\frac{c_{1} c_{2}}{c_{1}^{*} c_{2}^{*}}-\frac{c_{3}^{2}}{\left(c_{3}^{*}\right)^{2}}\right)\left(\begin{array}{c}
1 \\
1 \\
-2
\end{array}\right) \\
& \text { with } \delta_{1}^{*}=\left(c_{1}^{*} c_{3}^{*}\right)^{1 / 2}=\sigma \sqrt{3}, \delta_{2}^{*}=\left(c_{1}^{*} c_{2}^{*}\right)^{1 / 2} c_{3}=9 \sigma^{2}, \kappa_{1}=\sqrt{3}, \text { and } \kappa_{2}=1 .
\end{aligned}
$$




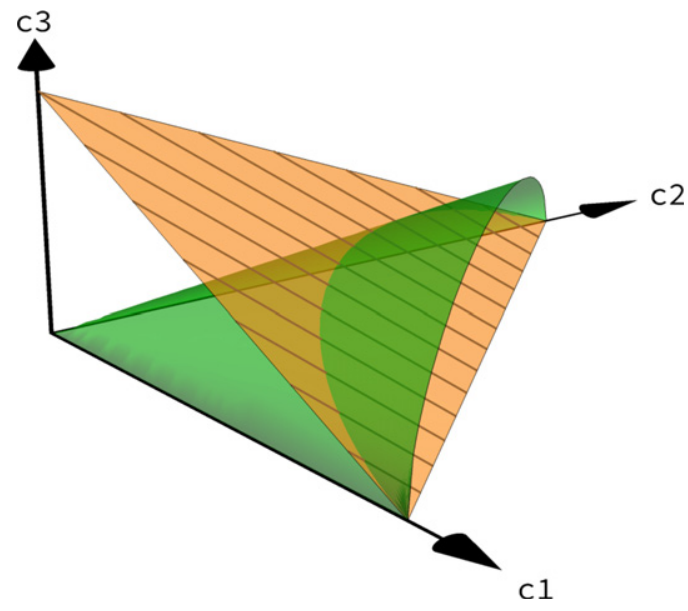

Figure 1. The state space $\mathbf{C}=\left[0, \infty\left[{ }^{3}\right.\right.$ decomposes into the triangles $Q c=c_{1}+c_{2}+$ $c_{3}=q$ (light brown), which decompose into the straight segments $Q_{\mathrm{fa}} c=\mathrm{q}$ (brown). Each segment has exactly one intersection with the fast equilibria $\mathscr{E}_{\mathrm{fa}}$ (green).

Thus, we find the cosh-type gradient structure $\left(\mathbf{C}, \mathcal{E}, \mathcal{R}_{\varepsilon}^{*}\right)$ of section 2.3 with

$$
\begin{gathered}
\mathcal{E}(c)=\sigma \lambda_{\mathrm{B}}\left(c_{1} / \sigma\right)+9 \sigma \lambda_{\mathrm{B}}\left(c_{2} /(9 \sigma)\right)+3 \sigma \lambda_{\mathrm{B}}\left(c_{3} /(3 \sigma)\right), \\
\mathcal{R}_{\varepsilon}^{*}(c, \xi)=\sqrt{3 c_{1} c_{3}} \mathbf{C}^{*}\left(\xi_{1}-\xi_{3}\right)+\frac{1}{\varepsilon} \sqrt{c_{1} c_{2} c_{3}^{2}} \mathrm{C}^{*}\left(\xi_{1}+\xi_{2}-2 \xi_{3}\right) .
\end{gathered}
$$

As noted just after proposition 2.3, $\mathcal{R}_{\varepsilon}^{*}$ is independent of $c_{*}$.

The associated fast system consists simply of one reaction, hence we find

$$
\Gamma_{\mathrm{fa}}=\operatorname{span}(1,1,-2)^{\top}, \quad Q_{\mathrm{fa}}=\left(\begin{array}{ccc}
1 & 1 & 1 \\
1 & -1 & 0
\end{array}\right), \quad \mathbf{Q}=\left\{\mathbf{q} \in \mathbb{R}^{2} \mid \mathrm{q}_{1} \geqslant 0\right\} .
$$

The stoichiometric sets $\mathbf{C}_{q}$ with $Q c=q \in \mathbb{R}^{1}$ are triangles, which decompose into the straight segments $\mathbf{C}_{\mathrm{q}}^{\mathrm{fa}}$ given by $Q_{\mathrm{fa}} c=\mathrm{q}$, whereas the set of fast equilibria

$$
\mathscr{E}_{\mathrm{fa}}=\left\{c \in \mathbf{C} \mid c_{1} c_{2}=c_{3}^{2}\right\} .
$$

is curved. See figure 1 for an illustration.

Finally, we discuss the evolution for our example starting with the initial condition $c_{0}=$ $(10,4,0)^{\top}$ such that $Q c^{\varepsilon}(t)=c_{1}^{\varepsilon}(t)+c_{2}^{\varepsilon}(t)+c_{3}^{\varepsilon}(t)=14$ is the conserved quantity. Since there is only one fast reaction, the second conserved quantity $c_{1}-c_{2}=\mathrm{q}_{2}=6$ shows that $\widetilde{c}^{\varepsilon}(\tau)=$ $c^{\varepsilon}(\varepsilon t)$ converges to $\widetilde{c}(\tau)$ and $\widetilde{c}(\tau) \rightarrow \bar{c}_{0}=(8,2,4)^{\top} \in \mathscr{E}_{\mathrm{fa}}$ for $\tau \rightarrow \infty$. 

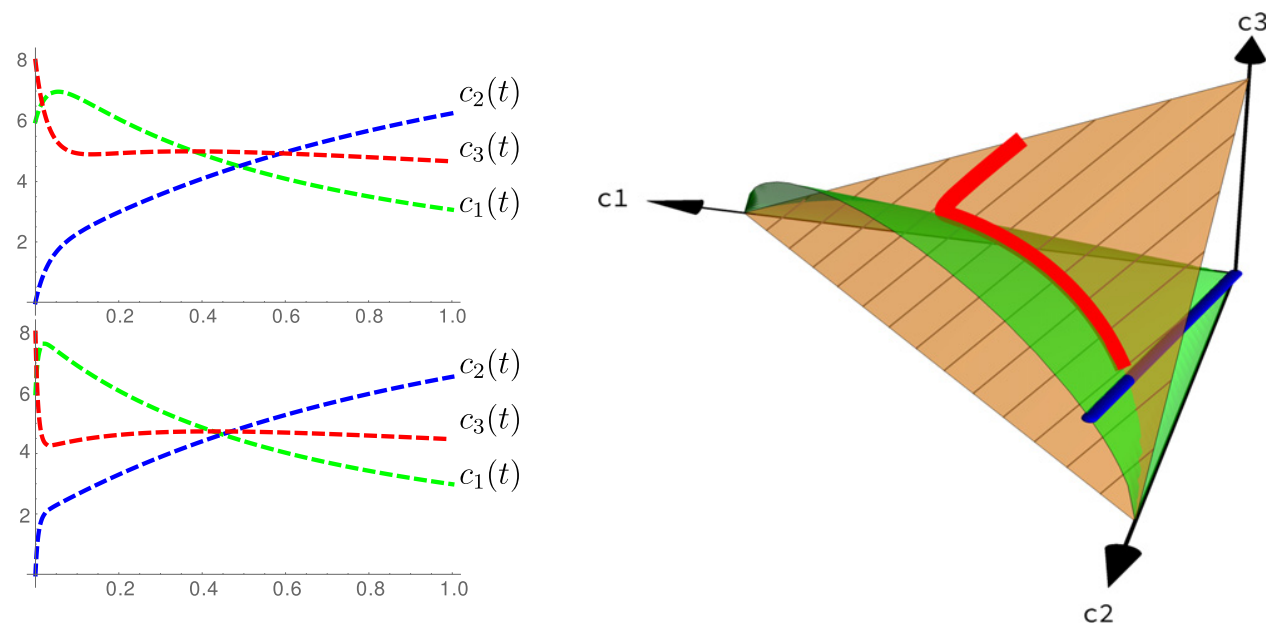

Figure 2. Numerical calculation of the solutions $c^{\varepsilon}(t)$ for the RRE (2.22) with $c^{\varepsilon}(0)=$ $(4,0,10)^{\top}$ with $\varepsilon=1$ (upper left) and $\varepsilon=0.2$ (lower left). The lower left figure shows the fast convergence to $\bar{c}_{0}=(8,2,4)^{\top}$. The right graphs displays the curve $t \mapsto c^{\varepsilon}(t)$ (red), which lies in the plane $Q c=14$ (light brown). It quickly approaches $\mathscr{M}_{\text {sl }}$ (green) and then moves towards the set of steady states (blue).

Thus, the limit solution $c^{0}$ satisfies the limiting equation (2.21), which reads in our case

$$
\begin{array}{r}
\dot{c}=\left(c_{3}-3 c_{1}\right)\left(\begin{array}{c}
1 \\
0 \\
-1
\end{array}\right)+\lambda_{0}\left(\begin{array}{c}
1 \\
1 \\
-2
\end{array}\right), \\
c_{1}(t) c_{2}(t)=c_{3}(t)^{2}, \quad c(0)=\bar{c}_{0}=(8,2,4)^{\top} .
\end{array}
$$

By eliminating the Lagrange multiplier $\lambda_{0} \in \mathbb{R}$ and using the conserved quantity $Q c=14$ this system is equivalent to the system

$$
\dot{c}_{1}-\dot{c}_{2}=c_{3}-3 c_{1}, \quad c_{1} c_{2}=c_{3}^{2}, \quad c_{1}+c_{2}+c_{3}=14 .
$$

Simulations are shown in figure 2 , which show the fast convergence to $\mathscr{E}_{\mathrm{fa}}$ and then the slow convergence to the final steady state $c_{\mathrm{eq}}=(1,9,4)^{\top}$.

\section{EDP-convergence and effective gradient structure}

In this section we first provide the precise definitions of EDP-convergence for GSs. Next we present the our main result concerning the EDP-limit of the cosh-type gradient structure for the fast-slow RRE with detailed-balance condition as introduced in section 2.4, where the proofs are postponed to later sections. Finally, in section 4 we discuss the obtained effective GS $\left(\mathbf{C}, \mathcal{E}, \mathcal{R}_{\text {eff }}\right)$ and show that the induced gradient-flow equation indeed is the same as the limiting equation (2.21).

\subsection{Definition of different types of EDP-convergence}

The definition of EDP-convergence for GSs relies on the notion of $\Gamma$-convergence for functionals (cf [Da193]). If $Y$ is a Banach space and $I_{\varepsilon}: Y \rightarrow \mathbb{R}_{\infty}$ we write $I_{\varepsilon} \stackrel{\Gamma}{\rightarrow} I_{0}$ for $\Gamma$-convergence 
in the strong topology, which is defined via the liminf and limsup estimates:

$$
\begin{gathered}
\Gamma-\liminf : \quad w_{\varepsilon} \rightarrow w_{0} \Longrightarrow \liminf _{\varepsilon \rightarrow 0^{+}} I_{\varepsilon}\left(w_{\varepsilon}\right) \geqslant I_{0}\left(w_{0}\right), \\
\Gamma-\lim \sup : \quad \forall \widehat{w}_{0} \in Y \exists\left(\widehat{w}_{\varepsilon}\right)_{\varepsilon}:\left(\widehat{w}_{\varepsilon} \rightarrow \widehat{w}_{0} \text { and } \limsup _{\varepsilon \rightarrow 0^{+}} I_{\varepsilon}\left(\widehat{w}_{\varepsilon}\right) \leqslant I_{0}\left(\widehat{w}_{0}\right)\right) .
\end{gathered}
$$

If in both conditions the strong convergence $\rightarrow$ is replaced by weak convergence $\rightarrow$, then we have (sequential) weak $\Gamma$-convergence and write $I_{\varepsilon} \stackrel{\Gamma}{\rightarrow} I_{0}$. If weak and strong $\Gamma$-convergence holds, this is called Mosco convergence and written as $I_{\varepsilon} \stackrel{\mathrm{M}}{\longrightarrow} I_{0}$.

For families of GSs $\left(X, \mathcal{E}_{\varepsilon}, \mathcal{R}_{\varepsilon}\right)$, three different levels of EDP-convergence are introduced and discussed in [DFM19, MMP21], called simple EDP-convergence, EDP-convergence with tilting, and contact EDP-convergence with tilting. Here we will only use the first two notions. For all three notions the choice of weak or strong topology is still to be decided according to the specific problem. Here in the state space $X=\mathbb{R}^{i_{*}}$ this question is irrelevant, but it is relevant for curves $u:[0, T] \rightarrow \mathbf{X}$ lying in $Y=L^{1}([0, T] ; X)$, where the state space $\mathbf{X}$ is a closed convex subset with non-empty interior of the Banach space $X$. For our paper, the strong topology will be sufficient.

Definition 3.1 (Simple EDP-convergence). A family of gradient structures $\left(\mathbf{X}, \mathcal{E}_{\varepsilon}, \mathcal{R}_{\varepsilon}\right)$ is said to EDP-converge to the GS $\left(\mathbf{X}, \mathcal{E}_{0}, \mathcal{R}_{\text {eff }}\right)$ if the following conditions hold:

(a) $\mathcal{E}_{\varepsilon} \stackrel{\Gamma}{\longrightarrow} \mathcal{E}_{0}$ on $\mathbf{X} \subset X$;

(b) $\mathfrak{D}_{\varepsilon}$ strongly $\Gamma$-converges to $\mathfrak{D}_{0}$ on $\mathrm{L}^{1}([0, T]$; $\mathbf{X})$ conditioned to bounded energies (we write $\mathfrak{D}_{\varepsilon} \stackrel{\Gamma_{\mathrm{E}}}{\longrightarrow} \mathfrak{D}_{0}$ ), i.e. we have

1. (Liminf) For all strongly converging families $u_{\varepsilon} \rightarrow u$ in $\mathrm{L}^{1}([0, T]$; X) which satisfy $\sup _{\varepsilon>0} \operatorname{ess~sup}_{t \in[0, T]} \mathcal{E}_{\varepsilon}\left(u_{\varepsilon}(t)\right)<\infty$, we have $\liminf \inf _{\varepsilon \rightarrow 0} \mathfrak{D}_{\varepsilon}\left(u_{\varepsilon}\right) \geqslant \mathfrak{D}_{0}(u)$.

2. (Limsup) For all $\widetilde{u} \in \mathrm{L}^{1}([0, T] ; \mathbf{X})$ there exists a strongly converging family $\widetilde{u}_{\varepsilon} \rightarrow$ $\widetilde{u}$ in $\mathrm{L}^{1}([0, T] ; \mathbf{X})$ with $\sup _{\varepsilon>0} \operatorname{ess} \sup _{t \in[0, T]} \mathcal{E}_{\varepsilon}\left(\widetilde{u}_{\varepsilon}(t)\right)<\infty$ and $\lim \sup _{\varepsilon \rightarrow 0^{+}} \mathfrak{D}_{\varepsilon}\left(\widetilde{u}_{\varepsilon}\right) \leqslant$ $\mathfrak{D}_{0}(\widetilde{u})$;

(c) There is an effective dissipation potential $\mathcal{R}_{\text {eff }}: \mathbf{X} \times X \rightarrow \mathbb{R}_{\infty}$ such that $\mathfrak{D}_{0}$ takes the form of a dual sum, namely $\mathfrak{D}_{0}(u)=\int_{0}^{T}\left\{\mathcal{R}_{\text {eff }}(u, \dot{u})+\mathcal{R}_{\text {eff }}^{*}\left(u,-\mathrm{D} \mathcal{E}_{\text {eff }}(u)\right)\right\} \mathrm{d} t$.

Similarly, one can also use weak $\Gamma$ or Mosco convergence conditioned to bounded energy, which we will then write as $\mathfrak{D}_{\varepsilon} \stackrel{\Gamma_{\mathrm{E}}}{\longrightarrow} \mathfrak{D}_{0}$ and $\mathfrak{D}_{\varepsilon} \stackrel{\mathrm{M}_{\mathrm{E}}}{\longrightarrow} \mathfrak{D}_{0}$. In fact, for our fast-slow reaction systems we are going to prove $\mathfrak{D}_{\varepsilon} \stackrel{\mathrm{M}_{\mathrm{E}}}{\longrightarrow} \mathfrak{D}_{0}$.

A general feature of EDP-convergence is that under suitable conditions the gradientflow equation $\dot{u}=\partial_{\xi} \mathcal{R}_{\text {eff }}^{*}\left(u,-\mathcal{D E}_{0}(u)\right)$ of the effective $\mathrm{GS}\left(X, \mathcal{E}_{0}, \mathcal{R}_{\text {eff }}\right)$ is indeed the limiting equation for the family $\dot{u}=\partial_{\xi} \mathcal{R}_{\varepsilon}^{*}\left(u,-\mathrm{D} \mathcal{E}_{\varepsilon}(u)\right)$, i.e. limits $u^{0}$ of solutions $u^{\varepsilon}$ of latter equations solve the former equation, see e.g. [Bra14, theorem 11.3], [MiS19, lemma 3.4], or [MMP21, lemma 2.8]. For our case, because of the degeneracy of the fast variables, such a result requires an assumption that the initial states $u_{\varepsilon}(0)$ converge to the slow manifold as $\varepsilon \rightarrow 0$. Propositions 4.3 and 4.4 describe this in detail.

A strengthening of simple EDP-convergence is the so-called EDP-convergence with tilting. This notion involves the tilted energy functionals $\mathcal{E}_{\varepsilon}^{\eta}: \mathbf{X} \ni u \mapsto \mathcal{E}_{\varepsilon}(u)-\langle\eta, u\rangle$, where the tilt $\eta$ (also called forcing) varies through the whole dual space $X^{*}$. 
Definition 3.2 (EDP-convergence with tilting (cf [MMP21, definition 2.14])). A family of gradient structures $\left(\mathbf{X}, \mathcal{E}_{\varepsilon}, \mathcal{R}_{\varepsilon}\right)$ is said to EDP-converge with tilting to the GS (X, $\left.\mathcal{E}_{0}, \mathcal{R}_{\text {eff }}\right)$, if for all tilts $\eta \in X^{*}$ we have $\left(\mathbf{X}, \mathcal{E}_{\varepsilon}^{\eta}, \mathcal{R}_{\varepsilon}\right)$ EDP-converges to $\left(\mathbf{X}, \mathcal{E}_{\varepsilon}^{\eta}, \mathcal{R}_{\text {eff }}\right)$.

In [MMP21, section 2.4] the admissible tilts are chosen to be general $C^{1}$ functions leading to tilted families $\mathcal{E}_{\varepsilon}^{\mathcal{F}}=\mathcal{E}_{\varepsilon}+\mathcal{F}$. This choice was inevitable because there the underlying space $\mathbf{Q}$ was a manifold. In the present paper the underlying space $\mathbf{C}$ is a convex subset of a linear space $\mathbf{X}$ which allows for the simpler definition.

We observe that $\mathcal{E}_{\varepsilon} \stackrel{\Gamma}{\rightarrow} \mathcal{E}_{0}$ implies $\mathcal{E}_{\varepsilon}^{\eta} \stackrel{\Gamma}{\rightarrow} \mathcal{E}_{0}^{\eta}$ for all $\eta \in X^{*}$ (and similarly for weak $\Gamma$ convergence), since the linear tilt $u \mapsto-\langle\eta, u\rangle$ is weakly continuous. The main and nontrivial assumption is that additionally

$$
\mathfrak{D}_{\varepsilon}^{\eta}: u \mapsto \int_{0}^{T}\left\{\mathcal{R}_{\varepsilon}(u, \dot{u})+\mathcal{R}_{\varepsilon}^{*}\left(u, \eta-\mathrm{D} \mathcal{E}_{\varepsilon}(u)\right)\right\} \mathrm{d} t
$$

$\Gamma$-converges in $\mathrm{L}^{1}([0, T] ; \mathbf{X})$ to $\mathfrak{D}_{0}^{\eta}$ for all $\eta \in X^{*}$ and that this limit $\mathfrak{D}_{0}^{\eta}$ is given in dual-sum form with $\mathcal{R}_{\text {eff }}$ via

$$
\mathfrak{D}_{0}^{\eta}(u)=\int_{0}^{T}\left\{\mathcal{R}_{\text {eff }}(u, \dot{u})+\mathcal{R}_{\text {eff }}^{*}\left(u, \eta-\mathrm{D} \mathcal{E}_{\text {eff }}(u)\right)\right\} \mathrm{d} t .
$$

The main point is that $\mathcal{R}_{\text {eff }}$ remains independent of $\eta \in X^{*}$. We refer to [MMP21] for a discussion of this and the other two notions of EDP-convergence.

\subsection{Our main EDP-convergence result}

Since we have assumed that the stationary measure does not depend on $\varepsilon>0$, also the energy $\mathcal{E}_{\varepsilon}=\mathcal{E}$ is $\varepsilon$-independent. Since $\mathcal{E}$ is also convex and lower semicontinuous, we have the trivial Mosco convergence $\mathcal{E}_{\varepsilon} \stackrel{\mathrm{M}}{\longrightarrow} \mathcal{E}$.

To study the $\Gamma$-limit of the dissipation functionals $\mathfrak{D}_{\varepsilon}$ we first extend them to the space

$$
\mathrm{L}^{1}([0, T] ; \mathbf{C}):=\left\{c \in \mathrm{L}^{1}\left([0, T] ; \mathbb{R}^{i_{*}}\right) \mid c(t) \in \mathbf{C} \text { a.e. }\right\} .
$$

For this we also use the slope functions (where $\mathrm{xy} \in\{\mathrm{fa}, \mathrm{sl}\}$ )

$$
\begin{aligned}
\mathcal{S}_{\varepsilon}(c) & =\mathcal{S}_{\mathrm{sl}}(c)+\frac{1}{\varepsilon} \mathcal{S}_{\mathrm{fa}}(c) \quad \text { with } \\
\mathcal{S}_{x y}(c) & =\sum_{r \in R_{x y}} 2 \kappa_{r} \delta_{r}^{*}\left(\left(\frac{c^{\alpha^{r}}}{c_{*}^{\alpha^{r}}}\right)^{1 / 2}-\left(\frac{c^{\beta^{r}}}{c_{*}^{\beta^{r}}}\right)^{1 / 2}\right)^{2} .
\end{aligned}
$$

For $\varepsilon>0$ the dissipation functional $\mathfrak{D}_{\varepsilon}: \mathrm{L}^{1}([0, T] ; \mathbf{C}) \rightarrow[0, \infty]$ is now given by

$$
\mathfrak{D}_{\varepsilon}(c)= \begin{cases}\int_{0}^{T}\left\{\mathcal{R}_{\varepsilon}(c, \dot{c})+\mathcal{S}_{\varepsilon}(c)\right\} \mathrm{d} t & \text { for } c \in W^{1,1}([0, T] ; \mathbf{C}), \\ \infty & \text { otherwise. }\end{cases}
$$

We recall that the dual dissipation potentials are given by (with $\gamma^{r}=\alpha^{r}-\beta^{r}$ ) 


$$
\begin{aligned}
\mathcal{R}_{\varepsilon}^{*}(c, \xi) & =\mathcal{R}_{\mathrm{sl}}^{*}(c, \xi)+\frac{1}{\varepsilon} \mathcal{R}_{\mathrm{fa}}^{*}(c, \xi) \quad \text { with } \\
\mathcal{R}_{x y}^{*}(c, \xi) & =\sum_{r \in R_{x y}} \kappa_{r} \sqrt{c^{\alpha^{r}} c^{\beta^{r}}} \mathrm{C}^{*}\left(\gamma^{r} \cdot \xi\right) .
\end{aligned}
$$

Because $\mathcal{S}_{\mathrm{fa}}(c) \geqslant 0$ and $\mathcal{R}_{\mathrm{fa}}^{*}(c, \xi) \geqslant 0$ we observe that $\mathcal{S}_{\varepsilon}(c)$ and $\mathcal{R}_{\varepsilon}^{*}(c, \xi)$ are monotonously increasing for $\varepsilon \downarrow 0$. Thus, their $\Gamma$-limits exist and are equal to the pointwise limits, which are denoted by $\mathcal{S}_{0}$ and $\mathcal{R}_{0}^{*}$ respectively (this uses [Dal93, remark 5.5] and the continuity of $\mathcal{S}_{\text {fa }}$ and $\mathcal{R}_{\mathrm{fa}}^{*}$ ).

Using (2.13) for the fast system we know that for $c \in \mathbf{C}$ the three conditions $\boldsymbol{R}_{\mathrm{fa}}(c)=0$, $\mathcal{S}_{\mathrm{fa}}(c)=0$, and $c \in \mathscr{E}_{\mathrm{fa}}$ are equivalent. Hence, we conclude

$$
\lim _{\varepsilon \rightarrow 0^{+}} \mathcal{S}_{\varepsilon}(c)=: \mathcal{S}_{0}(c)=\mathcal{S}_{\mathrm{sl}}(c)+\chi_{\mathscr{E}_{\mathrm{fa}}}(c), \quad \text { where } \chi_{A}(b)= \begin{cases}0 & \text { for } b \in A, \\ \infty & \text { for } b \notin A\end{cases}
$$

Obviously, we have $\lim _{\varepsilon \rightarrow 0^{+}} \mathcal{R}_{\varepsilon}^{*}(c, \xi)=: \mathcal{R}_{0}^{*}(c, \xi)=0$ for $\xi \in \Gamma_{\mathrm{fa}}^{\perp}$ and for $c \in \mathbf{C}_{+}$we obtain $\mathcal{R}_{0}^{*}(c, \xi)=\infty$ for $\xi \notin \Gamma_{\text {fa }}^{\perp}$. Thus, we define the effective dual dissipation potential as

$$
\mathcal{R}_{\mathrm{eff}}^{*}(c, \xi)=\mathcal{R}_{\mathrm{sl}}^{*}(c, \xi)+\chi_{\Gamma_{\mathrm{fa}}^{\perp}}(\xi)
$$

Note that $\mathcal{R}_{\text {eff }}^{*}(c, \xi) \geqslant \mathcal{R}_{0}^{*}(c, \xi)$ where inequality may happen on the boundary of $\mathbf{C}$, e.g. at $c=0$. Nevertheless, we have the important relation

$$
\forall c \in \mathbf{C}_{+}: \mathcal{R}_{\mathrm{eff}}^{*}(c,-\mathrm{DE}(c))=\mathcal{S}_{0}(c):=\mathcal{S}_{\mathrm{sl}}(c)+\chi_{\mathscr{E}_{\mathrm{fa}}}(c)
$$

The primal effective dissipation potential $\mathcal{R}_{\text {eff }}$ is given by the Legendre-Fenchel transformation:

$$
\begin{aligned}
\mathcal{R}_{\mathrm{eff}}(c, v) & =\sup _{\xi \in \mathbb{R}^{i_{*}}}\left\{v \cdot \xi-\mathcal{R}_{\mathrm{eff}}^{*}(c, \xi)\right\}=\sup _{\xi \in \mathbb{R}^{i *}}\left\{v \cdot \xi-\mathcal{R}_{\mathrm{sl}}^{*}(c, \xi)-\chi_{\Gamma_{\mathrm{fa}}^{\perp}}(\xi)\right\} \\
& =\inf _{v_{1}+v_{2}=v}\left\{\mathcal{R}_{\mathrm{sl}}\left(c, v_{1}\right)+\chi_{\Gamma_{\mathrm{fa}}}\left(v_{2}\right)\right\}=\inf _{v_{2} \in \Gamma_{\mathrm{fa}}}\left\{\mathcal{R}_{\mathrm{sl}}\left(c, v-v_{2}\right)\right\},
\end{aligned}
$$

where we have used $\left(\chi_{\Gamma_{\mathrm{fa}}}\right)^{*}=\chi_{\Gamma_{\mathrm{fa}}^{\perp}}$ and the classical theorem on the Legendre-Fenchel transformation turning a sum into an infimal convolution (see [Att84, proposition 3.4]).

To state our main result we now impose a non-trivial structural assumption that is crucial for our result and its proof. An analogous condition on the uniqueness of equilibria in each stoichiometric subset $\mathbf{C}_{q}^{\mathrm{fa}}$ was used in [Mie17, equation (17)]. We believe that the theory of EDP-convergence can be studied without this assumption, but then one has to refine the results and the solution technique suitably, see the counterexample in remark 3.10.

Assumption 3.3 (Conditions on the fast equilibria $\mathscr{E}_{\mathrm{fa}}$ ). For all $\mathrm{q} \in \mathbf{Q}:=\left\{Q_{\mathrm{fa}} c \mid c\right.$ $\in \mathbf{C}\}$, there is exactly one equilibrium of $c^{\prime}=\boldsymbol{R}_{\mathrm{fa}}(c)$ in the invariant subset $\mathbf{C}_{\mathrm{q}}^{\mathrm{fa}}(\operatorname{cf}(2.19))$, i.e.

$$
\text { (UFEC) } \forall \mathrm{q} \in \mathbf{Q}: \quad \#\left(\mathbf{C}_{\mathrm{q}}^{\mathrm{fa}} \cap \mathscr{E}_{\mathrm{fa}}\right)=1 \text {, }
$$

which is called the UFEC. By $\Psi: \mathbf{Q} \rightarrow \mathbf{C}$ we denote the mapping such that $\{\Psi(\mathbf{q})\}=\mathbf{C}_{\mathbf{q}}^{\mathrm{fa}} \cap \mathscr{E}_{\text {fa }}$ for all $q \in \mathbf{Q}$. 
We further impose the following positivity assumption on $\Psi$ :

$\exists \overline{\mathbf{q}} \in \mathbf{Q} \forall \theta \in] 0,1] \forall \mathbf{q} \in \mathbf{Q} \forall i \in I: \quad \Psi(\mathbf{q}+\theta \overline{\mathbf{q}})_{i}>0$ and $\Psi(\mathbf{q}+\theta \overline{\mathbf{q}})_{i} \geqslant \Psi(\mathbf{q})_{i}$.

The positivity and monotonicity assumption (3.6) seems to be only technical and it is only used at one point, namely in step 1 in the proof of theorem 5.5. We expect that this assumption can be avoided by a more careful construction of recovery sequence.

In section 3.3 we will show that one of possibly several equilibria in $\mathbf{C}_{\mathrm{q}}^{\mathrm{fa}}$ is always given as the minimizer of $\mathcal{E}$ on $\mathbf{C}_{q}^{\mathrm{fa}}$. Thus, the assumption really means that this 'thermodynamic equilibrium' is the only steady state. Our main $\Gamma$-convergence result reads as follows.

Theorem 3.4 (Г-convergence). Consider a fast-slow DBRS $\left(A, B, c_{*}, \widehat{\kappa}^{\varepsilon}\right)$ as in (2.15) together with its cosh-type gradient structure $\left(\mathbf{C}, \mathcal{E}, \mathcal{R}_{\varepsilon}\right)$ as in proposition 2.3 and the dissipation functional $\mathfrak{D}_{\varepsilon}$ defined in (3.2). Moreover, let assumption 3.3 be satisfied.

Then we have $\mathfrak{D}_{\varepsilon} \stackrel{\mathrm{M}_{\mathrm{E}}}{\longrightarrow} \mathfrak{D}_{0}$ on $L^{1}([0, T], \mathbf{C})$ conditioned to bounded energies, where $\mathfrak{D}_{0}$ : $L^{1}([0, T] ; \mathbf{C}) \rightarrow[0, \infty]$ is defined as

$$
\mathfrak{D}_{0}(c):= \begin{cases}\int_{0}^{T}\left\{\mathcal{R}_{\mathrm{eff}}(c, \dot{c})+\mathcal{S}_{0}(c)\right\} \mathrm{d} t & \text { for } c \in C^{0}([0, T] ; \mathbf{C}) \text { and } Q_{\mathrm{fa}} c \in W^{1,1}\left([0, T] ; \mathbb{R}^{m_{\mathrm{fa}}}\right), \\ \infty & \text { otherwise, }\end{cases}
$$

where $\mathcal{R}_{\text {eff }}$ and $\mathcal{S}_{0}$ are defined in (3.4).

The proof of this result is the content of section 5 .

We emphasize that the integrand of $\mathfrak{D}_{0}$ is (i) degenerate (non-coercive) in $\dot{q}$ and (ii) singular (taking the value $\infty$ ). Concerning (i), we recall that the definition of $\mathcal{R}_{\text {eff }}$ in (3.4b) implies that $\mathcal{R}_{\text {eff }}(c, \cdot)$ vanishes on $\Gamma_{\mathrm{fa}}$. In fact, it is only possible to control the time derivative of

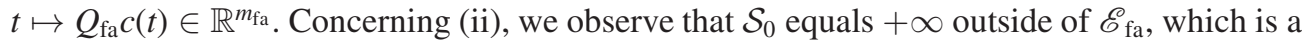
manifold of dimension $m_{\mathrm{fa}}$, and at each $c \in \mathscr{E}_{\mathrm{fa}} \cap \mathbf{C}_{+}$the subspaces $\mathrm{T}_{c} \mathscr{E}_{\mathrm{fa}}$ and $\Gamma_{\mathrm{fa}}$ are transversal, see section 4 . assumption 3.3 will be needed to avoid jump-type behavior which can occur otherwise, see the counterexample discussed in remark 3.10.

We now come to our main result on the EDP-convergence with tilting for the cosh-type GSs $\left(\mathbf{C}, \mathcal{E}, \mathcal{R}_{\varepsilon}\right)$ towards the effective $\mathrm{GS}\left(\mathbf{C}, \mathcal{E}, \mathcal{R}_{\text {eff }}\right)$.

The theorem enables to establish our main result on EDP-convergence with tilting. The result is a direct consequence of the $\Gamma$-convergence stated in theorem 3.4 and the general fact for the Boltzmann entropy that tilting is equivalent to changing the reference measure. In fact, introducing the relative Boltzmann entropy $\mathcal{H}(c \mid w)=\sum_{i=1}^{i_{*}} w_{i} \lambda_{\mathrm{B}}\left(c_{i} / w_{i}\right)$ we have $\mathcal{E}(c)=\mathcal{H}\left(c \mid c_{*}\right)$ and obtain, for all $\eta \in \mathbb{R}^{i_{*}}$, the relation

$$
\begin{aligned}
\mathcal{E}^{\eta}(c) & =\mathcal{E}(c)-\eta \cdot c=\mathcal{H}\left(c \mid \mathbb{D}^{\eta} c_{*}\right)+E_{\eta} \\
\text { with } \mathbb{D}^{\eta} c & :=\left(\mathrm{e}^{\eta_{i}} c_{i}\right)_{i \in I} \quad \text { and } \quad E_{\eta}=\sum_{i=1}^{i_{*}}\left(1-\mathrm{e}^{\eta_{i}}\right) c_{i}^{*} .
\end{aligned}
$$

Thus, we observe that tilting of a DBRS $\left(A, B, c_{*}, \widehat{\kappa}^{\varepsilon}\right)$ only changes the static property, namely the equilibrium $c_{*}$ into $\mathbb{D}^{\eta} c_{*}$, while the dissipative properties encoded in the stoichiometric matrices $A$ and $B$ and the reaction coefficients $\widehat{\kappa}$ remain unchanged.

Theorem 3.5 (EDP-convergence with tilting). Under the assumptions of theorem 3.4, the $G S s\left(\mathbf{C}, \mathcal{E}, \mathcal{R}_{\varepsilon}\right) E D P$-converge with tilting to the $G S\left(\mathbf{C}, \mathcal{E}, \mathcal{R}_{\text {eff }}\right)$.

Proof. Step 1. Simple EDP-convergence: since $\mathcal{E}_{\varepsilon}=\mathcal{E}$ is continuous we obviously have $\mathcal{E}_{\varepsilon} \stackrel{\mathrm{M}}{\longrightarrow} \mathcal{E}$. Moreover, theorem 3.4 provides $\mathfrak{D}_{\varepsilon} \stackrel{\mathrm{M}_{\mathrm{E}}}{\longrightarrow} \mathfrak{D}_{0}$. Finally, the relation (3.4a) shows that 
the integrand of $\mathfrak{D}_{0}$ has the desired dual structure $\mathcal{R}_{\text {eff }}(c, \dot{c})+\mathcal{R}_{\text {eff }}^{*}(c,-\mathrm{D} \mathcal{E}(c))$, where we used (3.4a). Thus, we have established the simple EDP-convergence of $\left(\mathbf{C}, \mathcal{E}, \mathcal{R}_{\varepsilon}\right)$ to the effective $\operatorname{GS}\left(\mathbf{C}, \mathcal{E}, \mathcal{R}_{\text {eff }}\right)$.

Step 2. EDP-convergence with tilting: we use that $\mathcal{E}^{\eta}=\mathcal{H}\left(\cdot \mid \mathbb{D}^{\eta} c_{*}\right)+E_{\eta}$ is of the same type as $\mathcal{E}=\mathcal{H}\left(\cdot \mid c_{*}\right)$ if we ignore the irrelevant constant energy shift. Clearly, the new fast-slow RRE (2.15) has the same $A, B, \kappa_{r}, i_{*}$, and hence $Q_{\text {fa }}$; only $c_{*}$ is replaced by $\mathbb{D}^{\eta} c_{*}$. Thus, all structural assumptions are the same, and theorem 3.4 is applicable for all $\eta \in \mathbb{R}^{i_{*}}$. In particular, the UFEC in (3.5) holds for the tilted DBRS by corollary 3.8. Thus, $\left(\mathbf{C}, \mathcal{E}^{\eta}, \mathcal{R}_{\varepsilon}\right)$ EDP-converges to $\left(\mathbf{C}, \mathcal{E}^{\eta}, \mathcal{R}_{\text {eff }}\right)$ according to step 1 . Since the effective dissipation potential $\mathcal{R}_{\text {eff }}$ is independent of $\eta \in \mathbb{R}^{i_{*}}$, we have shown EDP-convergence with tilting.

\subsection{Discussion of the UFEC and definition of $\mathscr{M}_{\mathrm{sl}}$}

Here we first prove properties of the function $\Psi$ that provides the fast equilibria (see assumption 3.3). Secondly, we show that UFEC is invariant under tilting.

The stoichiometric subsets $\mathbf{C}_{\mathrm{q}}^{\mathrm{fa}}:=\left\{c \in \mathbf{C} \mid Q_{\mathrm{fa}} c=\mathrm{q}\right\}$ are the intersection of the affine subspace $\left\{y \in \mathbb{R}^{i_{*}} \mid Q_{\mathrm{fa}} y=\mathrm{q}\right\}$ of dimension $m_{\mathrm{fa}}$ with the simplicial convex cone $\mathbf{C}=\left[0, \infty\left[{ }^{i_{*}}\right.\right.$. Hence, each $\mathbf{C}_{\mathrm{q}}^{\mathrm{fa}}$ is a closed and convex simplex of dimension $m(\mathbf{q}) \in\left\{0,1, \ldots, m_{\mathrm{fa}}\right\}$. The simplex-boundary $\partial \mathbf{C}_{\mathrm{q}}^{\mathrm{fa}}$ of such a simplex is the union of its boundary simplices of dimension $m(q)-1$. A two-dimensional $n$-gon has $n$ intervals as boundary, and an interval has 2 points as boundary. For the case of a point, which is the only zero-dimensional simplex, we say that the boundary is empty. We say that an equilibrium $c \in \mathscr{E}_{\mathrm{fa}}$ is a boundary equilibrium if $c \in \partial \mathbf{C}_{\mathrm{q}}^{\mathrm{fa}}$. Otherwise $c \in \mathscr{E}_{\text {fa }}$ is called an interior equilibrium.

The following result provides an alternative construction of the mapping $\Psi: \mathbf{Q} \rightarrow \mathbf{C}$ that is independent of the UFEC (3.5). We observe that $\Psi$ is defined for every fast DBRS $\left(A^{\mathrm{fa}}, B^{\mathrm{fa}}, c_{*}, \kappa^{\mathrm{fa}}\right)$ and that $\Psi$ only depends on $A^{\mathrm{fa}}-B^{\mathrm{fa}}$ and $c_{*}$. The first part of the next result is also shown in [MHM15, proposition 2.1] or [DLZ18, lemma 2.3].

Proposition 3.6 (Existence and continuity of interior equilibria). For a fast DBRS $\left(A^{\mathrm{fa}}, B^{\mathrm{fa}}, c_{*}, \kappa^{\mathrm{fa}}\right)$ the energy $\mathcal{E}$ only depends on $c_{*}$, and $Q_{\mathrm{fa}}$ only depends on $\Gamma_{\mathrm{fa}}=\operatorname{im}\left(A^{\mathrm{fa}}-B^{\mathrm{fa}}\right)$. For each $\mathbf{q} \in \mathbf{Q}$, denote by $\Psi(\mathrm{q})$ the unique minimizer of $\mathcal{E}$ on $\mathbf{C}_{\mathrm{q}}^{\mathrm{fa}}$. Then, $\Psi(\mathrm{q})$ is the only equilibrium of $\dot{c}=\boldsymbol{R}_{\mathrm{fa}}(c)$ that lies in the interior $\mathbf{C}_{\mathrm{q}}^{\mathrm{fa}} \backslash \partial \mathbf{C}_{\mathrm{q}}^{\mathrm{fa}}$. Moreover, the mapping $\Psi: \mathbf{Q} \rightarrow \mathbf{C}$ is continuous, and $\Psi$ : int $\mathbf{Q} \rightarrow$ int $\mathbf{C}$ is analytic.

Proof. Step 1. Uniqueness and existence of minimizer: the existence of a global minimizer follows from the coercivity of $\mathcal{E}$ and the closedness of $\mathbf{C}_{\mathrm{q}}^{\mathrm{fa}}$. The uniqueness follows from the convexity of $\mathbf{C}_{\mathrm{q}}^{\mathrm{fa}}$ and the strict convexity of $\mathcal{E}$.

Step 2. Interior property: if $\mathbf{C}_{\mathrm{q}}^{\mathrm{fa}}$ is a singleton $\{\widehat{c}\}$, then $\Psi(\mathrm{q})=\widehat{c}$ automatically lies in the interior. If $c^{\partial}$ is a point in the boundary and $c^{\circ}$ a point in the interior of $\mathbf{C}_{\mathrm{q}}^{\mathrm{fa}}$, then there is at least one $k \in I$ such that $c_{k}^{\partial}=0$ and $c_{k}^{\circ}>0$. Since $c_{k} \mapsto c_{k}^{*} \lambda_{\mathrm{B}}\left(c_{k} / c_{k}^{*}\right)$ has slope $-\infty$ at $c_{k}=0$, we conclude that $c^{\partial}$ cannot be a minimizer of $\mathcal{E}: c \mapsto \sum_{i} c_{i}^{*} \lambda_{\mathrm{B}}\left(c_{i} / c_{i}^{*}\right)$. Hence, $\widehat{c}=\Psi(\mathrm{q})$ lies in the interior of $\mathbf{C}_{\mathrm{q}}^{\mathrm{fa}}$.

Step 3. Unique equilibrium property: since $\mathcal{E}$ is a strict Liapunov function for the RRE, the minimizer $\Psi(\mathrm{q})$ has to be an equilibrium.

For the uniqueness, we consider first the case $\operatorname{dim}\left(\mathbf{C}_{\mathrm{q}}^{\mathrm{fa}}\right)=m_{\mathrm{fa}}$, in which case interior points in $\mathbf{C}_{\mathrm{q}}^{\mathrm{fa}}$ lie in $\mathbf{C}_{+}$. Hence, for any other equilibrium $c_{\mathrm{e}}$ in the interior of $\mathbf{C}_{\mathrm{q}}^{\mathrm{fa}}$ the derivative $\mathrm{D} \mathcal{E}\left(c_{\mathrm{e}}\right)=\left(\log \left(c_{i}^{\mathrm{e}} / c_{i}^{*}\right)\right)_{i}$ is well-defined. Moreover, lemma $2.4 \operatorname{implies} c_{\mathrm{e}}^{\alpha^{r}} / c_{*}^{\alpha^{r}}=c_{\mathrm{e}}^{\beta^{r}} / c_{*}^{\beta^{r}}$ for all $r \in R_{\mathrm{fa}}$. These two properties yield $\mathrm{D} \mathcal{E}\left(c_{\mathrm{e}}\right) \cdot \gamma^{r}=0$ for $r \in R_{\mathrm{fa}}$. But $\mathrm{DE}\left(c_{\mathrm{e}}\right) \in \Gamma_{\mathrm{fa}}^{\perp}$ and 
$\mathbf{C}_{\mathrm{q}}^{\mathrm{fa}} \subset c_{\mathrm{e}}+\Gamma_{\mathrm{fa}}$ guarantee that $c_{\mathrm{e}}$ minimizes the convex functional $\mathcal{E}$ on $\mathbf{C}_{\mathrm{q}}^{\mathrm{fa}}$, which yields $c_{\mathrm{e}}=\Psi(\mathrm{q})$.

If $\operatorname{dim}\left(\mathbf{C}_{\mathrm{q}}^{\mathrm{fa}}\right)=m(\mathrm{q})<m_{\mathrm{fa}}$ then there exists $I_{0} \subset I$ with $m_{\mathrm{fa}}-m(\mathbf{q})$ elements such that $\mathbf{C}_{\mathrm{q}}^{\mathrm{fa}} \subset$ $\left\{c \in \mathbf{C} \mid c_{i}=0\right.$ for all $\left.i \in I_{0}\right\}$ and that for interior points $\widetilde{c} \in \mathbf{C}_{\mathrm{q}}^{\mathrm{fa}} \backslash \partial \mathbf{C}_{\mathrm{q}}^{\mathrm{fa}}$ we have $\widetilde{c}_{i}>0$ for $i \notin I_{0}$. Hence, the above argument can be applied to the reduced system for $\widetilde{c}=\left(c_{i}\right)_{i \in I \backslash I_{0}}$, i.e. the components $c_{i}=0, i \in I_{0}$ are simply ignored.

Step 4. Continuity of $\Psi$ : consider a sequence $\mathrm{q}_{k} \rightarrow q_{\infty}$ and let $c_{k}=\Psi\left(\mathrm{q}_{k}\right)$, then we have to show that $c_{k} \rightarrow c_{\infty}$. We set $\alpha_{k}=\mathcal{E}\left(c_{k}\right)=\min \left\{\mathcal{E}(c) \mid c \in \mathbf{C}_{\mathrm{q}_{k}}^{\mathrm{fa}}\right\}$ and choose a subsequence $\left(k_{l}\right)$ such that $\alpha:=\liminf _{k \rightarrow \infty} \alpha_{k}=\lim _{l \rightarrow \infty} \alpha_{k_{l}}$. By coercivity of $\mathcal{E}$ we know that $\left(c_{k}\right)$ is bounded that there exists a further subsequence (not relabeled) with $c_{k_{l}} \rightarrow \widetilde{c}$ and $Q \widetilde{c}=\lim Q c_{k_{l}}=\lim \mathrm{q}_{k}=$ $\mathrm{q}_{\infty}$. Hence, we obtain the estimate

$$
\mathcal{E}\left(c_{\infty}\right) \leqslant \mathcal{E}(\widetilde{c})=\lim _{l \rightarrow \infty} \mathcal{E}\left(c_{k_{l}}\right)=\lim _{l \rightarrow \infty} \alpha_{k_{l}}=\alpha
$$

Moreover, our given $c_{\infty}$ and each $\varepsilon>0$ there exists a $\delta>0$ such that $Q\left(B_{\varepsilon}^{\mathbb{R}^{i_{*}}}\left(c_{\infty}\right) \cap \mathbf{C}\right)$ contains the set $B_{\delta}^{\mathbb{R}^{m}}\left(\mathrm{q}_{\infty}\right) \cap \mathbf{Q}$. Thus, we find a sequence $\left(\widehat{c}_{k}\right)_{k \in \mathbb{N}}$ with $\widehat{c}_{k} \rightarrow c_{\infty}$ and $Q \widehat{c}_{k}=\mathrm{q}_{k} \rightarrow$ $\mathrm{q}_{\infty}$. Since $\mathcal{E}$ is continuous we conclude

$$
\mathcal{E}\left(c_{\infty}\right)=\lim _{k \rightarrow \infty} \mathcal{E}\left(\widehat{c}_{k}\right) \geqslant \liminf _{k \rightarrow \infty} \mathcal{E}\left(c_{k}\right)=\alpha .
$$

With (3.8) we conclude $\mathcal{E}(\widetilde{c})=\mathcal{E}\left(c_{\infty}\right)$, which implies $c_{k} \rightarrow c_{\infty}=\Psi\left(\mathrm{q}_{\infty}\right)$, as desired.

Step 5. Analyticity of $\Psi$ : for $\mathbf{q} \in$ int $\mathbf{Q}$ we have $\Psi(\mathbf{q}) \in \mathbf{C}_{+}=$int $\mathbf{C}$. Hence, $c=\Psi(\mathbf{q})$ can be characterized by the Lagrange principle for constrained minimizers using the Lagrange function $L(c, \lambda)=\mathcal{E}(c)-\mu \cdot\left(Q_{\mathrm{fa}} c-\mathrm{q}\right)$ with $\mu \in \mathbb{R}^{m_{\mathrm{fa}}}$. This characterization leads to the equation $F(c, \mu)=(0, q)$, where

$$
F(c, \mu):=\left(\mathrm{D} \mathcal{E}(c)-Q_{\mathrm{fa}}^{\top} \mu, Q_{\mathrm{fa}} c\right) .
$$

Obviously, $F: \mathbf{C}_{+} \times \mathbb{R}^{m_{\mathrm{fa}}} \rightarrow \mathbb{R}^{i_{*}} \times \mathbb{R}^{m_{\mathrm{fa}}}$ is analytic, and we have $F(\Psi(\mathbf{q}), \tilde{\mu}(\mathbf{q}))=(0, \mathbf{q})$ for a suitable $\widetilde{\mu}$. If we can show that $\operatorname{DF}(\Psi(\mathrm{q}), \mu)$ is invertible for all $\mathbf{q} \in$ int $\mathbf{Q}$, then the implicit function theorem implies that the mapping $q \rightarrow(\Psi(q), \widetilde{\mu}(q))$ is analytic as well.

The Jacobian of $F(c, \mu)$ is given by $\mathrm{D} F(c, \mu)=\left(\begin{array}{cc}\mathrm{D}^{2} \mathcal{E}(c) & -Q_{\mathrm{fa}}^{\top} \\ Q_{\mathrm{fa}} & 0\end{array}\right)$, and we prove that $\mathrm{D} F(c, \mu)$ is invertible by showing that its kernel is trivial. Let $(w, \eta)$ be such that $\mathrm{D} F(c, \mu)(w, \eta)^{\top}=0$. We conclude that $\mathrm{D}^{2} \mathcal{E}(c) w=Q_{\mathrm{fa}}^{\top} \eta$ and $Q_{\mathrm{fa}} w=0$. Since $c$ is positive, the Hessian $\mathrm{D}^{2} \mathcal{E}(c)$ is invertible, and hence, we have $Q_{\mathrm{fa}} \mathrm{D}^{2} \mathcal{E}(c)^{-1} Q_{\mathrm{fa}}^{\top} \eta=0$. Multiplying $\eta$ from the left and using that $\mathrm{D}^{2} \mathcal{E}(c)^{-1}$ is a positive matrix, we have $Q_{\mathrm{fa}}^{\top} \eta=0$. Since $Q_{\mathrm{fa}}^{\top}$ is injective, we conclude that $\eta=0$ which implies that also $w=0$ due to $\mathrm{D}^{2} \mathcal{E}(c) w=Q_{\mathrm{fa}}^{\top} \eta=0$.

For later use we observe that by construction we have the relation

$$
Q_{\text {fa }} \Psi(\mathrm{q})=\mathrm{q} \quad \text { for all } \mathrm{q} \in \mathbf{Q} .
$$

A crucial role in our further analysis will be played by the image of $\Psi$, which we call the slow manifold:

$$
\mathscr{M}_{\mathrm{sl}}:=\operatorname{im}(\Psi)=\{\Psi(\mathrm{q}) \mid \mathrm{q} \in \mathbf{Q}\} \subset \mathbf{C},
$$

which is a closed set that is contained in the set of the fast equilibria $\mathscr{E}_{\text {fa }}$ defined in (2.20). The UFEC in (3.5) is made to guarantee that $\mathscr{M}_{\mathrm{sl}}$ contains all the fast equilibria:

$$
(\text { UFEC }) \Longleftrightarrow \mathscr{E}_{\mathrm{fa}}=\mathscr{M}_{\mathrm{sl}} .
$$



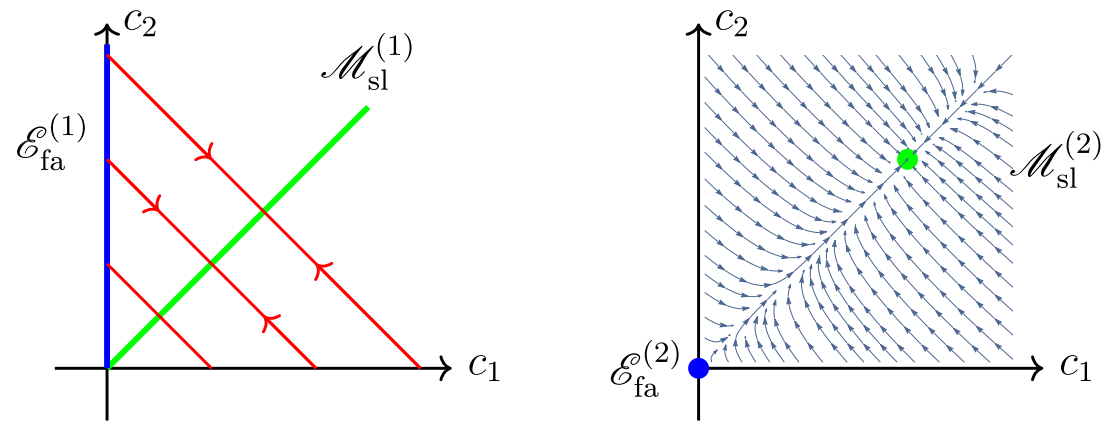

Figure 3. The slow manifolds $\mathscr{M}_{\mathrm{sl}}^{(k)}$ (green) are strictly contained in $\mathscr{E}_{\mathrm{fa}}^{(k)}$ (blue and green). The blue points are unstable, while the green points are stable. In the case (1) the invariant sets $\mathbf{C}_{\mathrm{q}}^{\mathrm{fa}}$ (red) are one-dimensional, while in case (2) we have $\mathbf{C}_{0}^{\mathrm{fa}}=\mathbf{C}$.

It is important to emphasize that $\mathscr{E}_{\text {fa }}$ can be strictly bigger than $\mathscr{M}_{\text {sl }}$, but by proposition 3.6 the equilibria in $\mathscr{E}_{\mathrm{fa}} \backslash \mathscr{M}_{\mathrm{sl}}$ must be so-called boundary equilibria, i.e. they lie in $\partial \mathbf{C}_{\mathrm{q}}^{\mathrm{fa}} \subset \partial \mathbf{C}$. (In the case that $\mathbf{C}_{\mathrm{q}}^{\mathrm{fa}} \subset \partial \mathbf{C}$ the equilibrium $\Psi(\mathrm{q})$ lies in the boundary of $\mathbf{C}$, but is not a boundary equilibrium!)

The equilibria on $\mathscr{M}_{\mathrm{sl}}$ are stable, since they are global minimizers of the Liapunov function $\mathcal{E}$ in their invariant subset. In contrast, possible boundary equilibria are always unstable, because starting near the equilibrium but in the interior of $\mathbf{C}_{\mathrm{q}}^{\mathrm{fa}}$ gives a solution moving towards $\Psi(q)$, see figure 3 . The UFEC may fail if one has autocatalytic reactions where the product $\alpha_{i}^{r} \beta_{i}^{r}$ is strictly positive for some $i \in I$; see the example treated in remark 3.9.

The following simple result provides the characterization of the slow manifold $\mathscr{M}_{\mathrm{sl}}$ in terms of the potential force $\mathrm{DE}(c)$ and the annihilator of the fast subspace $\Gamma_{\text {fa }}$.

Lemma 3.7. Consider a fast DBRS $\left(A^{\mathrm{fa}}, B^{\mathrm{fa}}, c_{*}, \kappa^{\mathrm{fa}}\right)$. Then for $c \in \mathbf{C}_{+}$we have

$$
\mathrm{DE}(c) \in \Gamma_{\mathrm{fa}}^{\perp}=\left\{\xi \in \mathbb{R}^{i_{*}} \mid \xi \cdot \gamma^{r} \quad \text { for } r \in R_{\mathrm{fa}}\right\} \quad \Longleftrightarrow c \in \mathscr{M}_{\mathrm{sl}} .
$$

Proof. Using $\mathrm{D} \mathcal{E}(c)=\left(\log \left(c_{i} / c_{i}^{*}\right)\right)_{i \in I}$ we find, for all $r \in R_{\mathrm{fa}}$,

$$
0=\mathrm{D} \mathcal{E}(c) \cdot \gamma^{r}=\log \left(\frac{c^{\alpha^{r}}}{c_{*}^{\alpha^{r}}} \frac{c_{*}^{\beta^{r}}}{c^{\beta^{r}}}\right) \Longleftrightarrow \frac{c^{\alpha^{r}}}{c_{*}^{\alpha^{r}}}=\frac{c^{\beta^{r}}}{c_{*}^{\beta^{r}}}
$$

With proposition 3.6 and the definition of $\mathscr{M}_{\text {sl }}$ in (3.10) we obtain the desired result.

Finally, we show that the UFEC is invariant under tilting. This is a nice consequence of the fact that tilting in systems satisfying the DBC allows us easily to follow the changes in the set $\mathscr{E}_{\text {fa }}$ of fast equilibria.

Corollary 3.8 (UFEC and tilting). Consider a fast DBRS $\left(A^{\mathrm{fa}}, B^{\mathrm{fa}}, c_{*}, \kappa^{\mathrm{fa}}\right)$ and general tilt vectors $\eta \in \mathbb{R}^{i_{*}}$. Denote by $\mathscr{E}_{\mathrm{fa}}$ and $\mathscr{M}_{\mathrm{sl}}^{\eta}$ the set of equilibria and the slow manifold, respectively, for the fast $D B R S\left(A^{\mathrm{fa}}, B^{\mathrm{fa}}, \mathbb{D}^{\eta} C_{*}, \kappa^{\mathrm{fa}}\right)$. Then, the following holds:

(a) $\mathscr{E}_{\mathrm{fa}}^{\eta}=\mathbb{D}^{\eta} \mathscr{E}_{\mathrm{fa}}^{0}$ and $\mathscr{M}_{\mathrm{sl}}^{\eta}=\mathbb{D}^{\eta} \mathscr{M}_{\mathrm{sl}}^{0}$,

(b) $\left(A^{\mathrm{fa}}, B^{\mathrm{fa}}, c_{*}, \kappa^{\mathrm{fa}}\right)$ satisfies UFEC if and only if $\left(A^{\mathrm{fa}}, B^{\mathrm{fa}}, \mathbb{D}^{\eta} c_{*}, \kappa^{\mathrm{fa}}\right)$ does so. 
Proof. By lemma 2.4 the equilibria $c \in \mathscr{E}_{\mathrm{fa}}^{0}$ are given by the condition $\frac{c^{\alpha^{r}}}{c_{*}^{\alpha^{r}}}=\frac{c^{\beta^{r}}}{c_{*}^{\beta^{r}}}$ for all $r \in$ $R_{\text {fa. }}$. However, changing $c$ and $c_{*}$ into $\mathbb{D}^{\eta} c$ and $\mathbb{D}^{\eta} c_{*}$, respectively, shows that the condition remains the same.

Moreover, for $c \in \mathbf{C}_{+}$we have $\mathrm{DE}^{\eta}\left(\mathbb{D}^{\eta} c\right)=\mathrm{D} \mathcal{E}(c)$ by construction. Since $c \in \mathbf{C}_{+} \cap \mathscr{M}_{\mathrm{sl}}^{0}$ is equivalent to $\mathrm{DE}(c) \in \Gamma_{\mathrm{fa}}^{\perp}$ we have $\mathbb{D}^{\eta} c \in \mathscr{M}_{\mathrm{sl}}^{\eta}$. By the continuity of $\Psi=\Psi^{0}$ and $\Psi^{\eta}$ (see proposition 3.6) we conclude $\mathbb{D}^{\eta} \mathscr{M}_{\mathrm{sl}}^{0} \subset \mathscr{M}_{\mathrm{sl}}^{\eta}$. As $\mathbb{D}^{\eta}$ is invertible, we can revert the argument and arrive at $\mathbb{D}^{\eta} \mathscr{M}_{\mathrm{sl}}^{0}=\mathscr{M}_{\mathrm{sl}}^{\eta}$. Thus, (a) is established.

With (a) we see that $\mathscr{E}_{\mathrm{fa}}^{0}=\mathscr{M}_{\mathrm{sl}}^{0}$ is equivalent to $\mathscr{E}_{\mathrm{fa}}^{\eta}=\mathscr{M}_{\mathrm{sl}}^{\eta}$, and (b) is established as well.

\subsection{Examples and problems without the UFEC}

In the following two remarks, we firstly provide a few examples where UFEC does not hold and secondly show that our main result in theorem 3.4 fails without UFEC.

Remark 3.9 (Examples without UFEC). The simplest example of an RRE not satisfying the UFEC condition is the autocatalytic reaction $2 X \rightleftharpoons X$, leading to the RRE $\dot{c}=\frac{1}{\varepsilon}\left(c-c^{2}\right)$, where $\Gamma=\Gamma_{\mathrm{fa}}=\mathbb{R}$ and $m=m_{\mathrm{fa}}=0$. In particular, the fast stoichiometric subset $\mathbf{C}=\mathbf{C}_{0}^{\mathrm{fa}}=$ $\left[0, \infty\right.$ [ contains the interior equilibrium $c_{*}=1$ and the boundary equilibrium $c=0$.

Next, we consider two different fast systems for two species, the first with the single autocatalytic reaction $X_{1}+X_{2} \leftrightharpoons 2 X_{1}$ and the second with two non-autocatalytic reactions $2 X_{1} \leftrightharpoons X_{2}$ and $X_{1} \leftrightharpoons 2 X_{2}$. The fast RREs read

$$
\begin{aligned}
& c^{\prime}=\boldsymbol{R}^{(1)}(c)=\left(c_{1}^{2}-c_{1} c_{2}\right)\left(\begin{array}{c}
-1 \\
1
\end{array}\right), \\
& c^{\prime}=\boldsymbol{R}^{(2)}(c)=\left(c_{1}^{2}-c_{2}\right)\left(\begin{array}{c}
-2 \\
1
\end{array}\right)+\left(c_{1}-c_{2}^{2}\right)\left(\begin{array}{c}
-1 \\
2
\end{array}\right) .
\end{aligned}
$$

The conserved quantities are given by the matrices

$$
Q_{\mathrm{fa}}^{(1)} c=c_{1}+c_{2} \in \mathbf{Q}^{(1)}=\left[0, \infty\left[\text { and } \quad Q_{\mathrm{fa}}^{(2)} c=0 \in \mathbf{Q}^{(2)}=\{0\} .\right.\right.
$$

The functions $\Psi$ for the minimizers of $\mathcal{E}$ over $\mathbf{C}_{\mathbf{q}}^{\text {fa }}$ are given by $\Psi^{(1)}(\mathbf{q})=(\mathbf{q} / 2, \mathbf{q} / 2)^{\top}$ and $\Psi^{(2)}(0)=(1,1)^{\top}$ leading to

$$
\mathscr{M}_{\mathrm{sl}}^{(1)}=\left\{(z, z)^{\top} \mid z \geqslant 0\right\} \quad \text { and } \quad \mathscr{M}_{\mathrm{sl}}^{(2)}=\left\{(1,1)^{\top}\right\} .
$$

However, the set of fast equilibria is bigger in both cases:

$$
\mathscr{E}_{\mathrm{fa}}^{(1)}=\mathscr{M}_{\mathrm{sl}}^{(1)} \dot{\cup}\{(0, z) \mid z \geqslant 0\} \quad \text { and } \quad \mathscr{E}_{\mathrm{fa}}^{(2)}=\mathscr{M}_{\mathrm{sl}}^{(2)} \dot{\cup}\left\{(0,0)^{\top}\right\} .
$$

Figure 3 displays the invariant sets $\mathbf{C}_{\mathrm{q}}^{\mathrm{fa}}, \mathscr{M}_{\mathrm{sl}}$, and $\mathscr{E}_{\text {fa }}$ for both cases.

To the knowledge of the authors there are currently no general sufficient conditions on the fast DBRS $\left(A^{\mathrm{fa}}, B^{\mathrm{fa}}, c_{*}, \kappa^{\mathrm{fa}}\right)$ available that guarantee the validity of the UFEC. However, in many applications the number $\#\left(R_{\mathrm{fa}}\right)$ of fast reactions is rather small such that an analysis of the fast RRE is easily done.

The next remark shows that theorem 3.4 does not hold if the UFEC in (3.5) does not hold.

Remark 3.10 (A counterexample with jumps). We return to the first RRE $\dot{c}=\frac{1}{\varepsilon}(c-$ $c^{2}$ ) of the previous remark. The associated dissipation potential and slope functions are

$$
\mathcal{R}_{\varepsilon}^{*}(c, \xi)=\frac{c^{3 / 2}}{\varepsilon} \mathrm{C}^{*}(\xi) \quad \text { and } \quad \mathcal{S}_{\varepsilon}(c)=\frac{2}{\varepsilon} c\left(c^{1 / 2}-1\right)^{2}
$$


Moreover, the dissipation functional $\mathfrak{D}_{\varepsilon}$ takes the form

$$
\left.\mathfrak{D}_{\varepsilon}(c)=\int_{0}^{T}\left\{\frac{c^{3 / 2}}{\varepsilon} \mathrm{C}\left(\frac{\varepsilon \dot{c}}{c^{3 / 2}}\right)+\frac{2 c}{\varepsilon}\left(c^{1 / 2}-1\right)^{2}\right)\right\} \mathrm{d} t .
$$

Note that $c \equiv 0$ and $c \equiv 1$ yield $\mathfrak{D}_{\varepsilon}(c)=0$. Moreover, fixing $\left.t_{*} \in\right] 0, T\left[\right.$ the trajectories $\widetilde{c}^{\varepsilon}(t)=$ $\mathrm{e}^{\left(t-t_{*}\right) / \varepsilon} /\left(1+\mathrm{e}^{\left(t-t_{*}\right) / \varepsilon}\right)$ are exact solutions of the RRE $\dot{c}=\frac{1}{\varepsilon}\left(c-c^{2}\right)$, hence the EDP gives $\mathfrak{D}_{\varepsilon}\left(\widetilde{c}^{\varepsilon}\right)=\mathcal{E}\left(\widetilde{c}^{\varepsilon}(0)\right)-\mathcal{E}\left(\widetilde{c}^{\varepsilon}(T)\right) \leqslant \mathcal{E}(0)-\mathcal{E}(1)=1$. Thus, the limit function $\widetilde{c}^{0}$ with $\widehat{c}^{0}(t)=0$ for $t<t_{*}$ and $\widehat{c}^{0}(t)=1$ for $t>t_{*}$ is not continuous but must satisfy $\mathfrak{D}_{0}\left(\widetilde{c}^{0}\right) \leqslant 1$, which is in contradiction to theorem 3.4 .

Indeed, using the Modica-Mortola approach as described in [Bra02, section 6] (involving the estimate $\left.\mathcal{R}_{\varepsilon}(c, \dot{c})+\mathcal{R}_{\varepsilon}^{*}(c,-\mathrm{D} \mathcal{E}(c)) \geqslant-\mathrm{D} \mathcal{E}(c) \dot{c}\right)$ it can be shown that $\mathfrak{D}_{\varepsilon} \stackrel{\Gamma}{\rightarrow} \mathfrak{D}_{0}$ in $\mathrm{L}^{1}([0, T] ; \mathbb{R})$, where $\mathfrak{D}_{0}$ is finite only on piecewise constant functions taking values in $\{0,1\}$ only. Moreover, for these functions $\mathfrak{D}_{0}(c)$ equals the number of jumps times $\mathcal{E}(0)-\mathcal{E}(1)=1$. The same was also observed in [Ste19].

\section{The effective GS and the limiting equation}

Here we present two different ways to derive the limiting equation from our effective GS. The first one is in line with the coarse-graining approach developed in [MiS19], where a lowerdimensional system is derived for the coarse-grained variable $\mathrm{q}=Q_{\mathrm{fa}} c$ and the restriction $c=$ $\Psi(q)$ is built into the model. The second one follows [Bot03] and [DLZ18, theorem 4.5], where the variable $c$ is maintained and the constraint $c \in \mathscr{M}_{\mathrm{sl}}$ is realized by a suitable projection.

In both cases we start from the $\Gamma$-limit $\mathfrak{D}_{0}$ of the dissipation functionals $\mathfrak{D}_{\varepsilon}$. Combining the $\Gamma$-convergence of $\mathfrak{D}_{\varepsilon}$ and an assumption of well-preparedness of the initial data, we can take the limit in the EDP to find

$$
\begin{aligned}
\mathcal{E}(c(T))+\mathfrak{D}_{0}(c ; 0, T) & \leqslant \mathcal{E}(c(0)) \\
\text { with } \mathfrak{D}_{0}(c ; 0, T) & =\int_{0}^{T}\left\{\mathcal{R}_{\mathrm{eff}}(c, \dot{c})+\mathcal{S}_{0}(c)\right\} \mathrm{d} t .
\end{aligned}
$$

From this inequality we recover the limiting evolution by theorem 2.1.

Any solution satisfies the condition

$$
\int_{0}^{T} \mathcal{S}_{0}(c(t)) \mathrm{d} t \leqslant \mathfrak{D}_{0}(c ; 0, T) \leqslant \mathcal{E}(c(0))-\mathcal{E}(c(T))<\infty,
$$

where by the UFEC the function $\mathcal{S}_{0}$ assumes the value $+\infty$ for $c \notin \mathscr{M}_{\text {sl }}$. Hence, the continuity of $c$ implies that $c(t) \in \mathscr{M}_{\mathrm{sl}}$ for all $t \in[0, T]$. Thus, setting $\mathrm{q}(t):=Q_{\mathrm{fa}} c(t)$ and using the relation (3.9) we have $c(t)=\Psi(\mathrm{q}(t))$ for all $t \in[0, T]$. We recall that the properties

$$
c \in C^{0}([0, T] ; \mathbf{C}) \quad \text { and } \quad \mathrm{q}=Q_{\mathrm{fa}} c \in W^{1,1}\left([0, T] ; \mathbb{R}^{m_{\mathrm{fa}}}\right)
$$

are consequences of theorem 3.4.

\subsection{Coarse-graining approach}

In this part we concentrate solely on the slow variables $q$ and define

$$
\mathrm{E}(\mathrm{q}):=\mathcal{E}(\Psi(\mathrm{q})) \quad \text { and } \quad \mathrm{R}^{*}(\mathrm{q}, \zeta):=\mathcal{R}_{\mathrm{sl}}^{*}\left(\Psi(\mathrm{q}), Q_{\mathrm{fa}}^{\top} \zeta\right)
$$


which defines a reduced GS $(\mathbf{Q}, E, R)$ for the coarse-grained state $q \in \mathbf{Q} \subset \mathbb{R}^{m_{\mathrm{fa}}}$. In particular, $\mathrm{R}^{*}: \mathbf{Q} \times \mathbb{R}^{m_{\mathrm{fa}}} \rightarrow[0, \infty]$ is a well-defined dual dissipation potential as $Q_{\mathrm{fa}}^{\top}: \mathbb{R}^{m_{\mathrm{fa}}} \rightarrow \mathbb{R}^{i_{*}}$.

The main result of this subsection will be that the gradient-flow equation for the reduced GS $(\mathbf{Q}, E, R)$ is indeed the limiting equation and it has a simple representation in terms of $\boldsymbol{R}_{\mathrm{sl}}$, $Q_{\text {fa }}$, and $\Psi$ :

$$
\dot{\mathrm{q}}=\partial_{\zeta} \mathrm{R}^{*}(\mathrm{q},-\mathrm{DE}(\mathrm{q}))=Q_{\mathrm{fa}} \boldsymbol{R}_{\mathrm{sl}}(\Psi(q)) .
$$

Thus, (Q, E, R) provides an exact nonlinear coarse-graining in the sense of [2020, section 6.1], where the relation $I_{m_{\mathrm{fa}}}=Q_{\mathrm{fa}} \mathrm{D} \Psi(\mathrm{q})$ simplifies the formula for $\mathrm{R}^{*}$ compared to [2020, equation (6.2)].

Remark 4.1. This theory is a nonlinear generalization of the coarse-graining theory developed in [MiS19], where $\dot{\widehat{c}}=M A_{\mathrm{sl}} N \widehat{c}$ is the coarse-grained equation. In our case the role of the reconstruction operator $N: \mathbb{R}^{J} \rightarrow \mathbb{R}^{I}$ is played by the nonlinear mapping $\Psi: \mathbf{Q} \rightarrow \mathbf{C}$, while the role of the coarse-graining operator $M: \mathbb{R}^{I} \rightarrow \mathbb{R}^{J}$ is our linear operator $Q_{\mathrm{fa}}: \mathbf{C} \rightarrow \mathbf{Q}$.

The following result provides first the justification of the second identity in (4.3), and then shows that this equation is indeed the limiting equation obtained from the EDP for $\mathcal{E}$ and $\mathfrak{D}_{0}$.

Proposition 4.2 (Reduced gradient structure). Let the $\operatorname{DBRS}\left(A, B, c_{*}, \widehat{\kappa}^{\varepsilon}\right)$ be given as in section 2.4 and satisfy the $\operatorname{UFEC}(3.5)$, and let $\left(\mathbf{Q}, \mathrm{E}, \mathrm{R}^{*}\right)$ be defined as above. Then the following identities are valid:

(a) For $\mathbf{q} \in$ int $\mathbf{Q}$ we have

$$
Q_{\mathrm{fa}} \mathrm{D} \Psi(\mathrm{q})=I_{m_{\mathrm{fa}}}, \quad \text { and } \quad Q_{\mathrm{fa}}^{\top} \mathrm{D} \Psi(\mathrm{q})^{\top} \text { is a projection onto } \mathrm{im}\left(Q_{\mathrm{fa}}^{\top}\right)=\Gamma_{\mathrm{fa}}^{\perp} ;
$$

(b) For $\mathbf{q} \in$ int $\mathbf{Q}$ we have $\partial_{\zeta} \mathrm{R}^{*}(\mathrm{q},-\mathrm{DE}(\mathrm{q}))=Q_{\mathrm{fa}} \boldsymbol{R}_{\mathrm{sl}}(\Psi(q))$;

(c) The primal dissipation potential $\mathrm{R}$ takes the form

$$
\mathrm{R}(\mathbf{q}, w)=\inf \left\{\mathcal{R}_{\mathrm{sl}}(\Psi(\mathbf{q}), v) \mid Q_{\mathrm{fa}} v=w\right\}=\mathcal{R}_{\mathrm{eff}}(\Psi(\mathbf{q}), \widetilde{v}) \text { whenever } Q_{\mathrm{fa}} \widetilde{v}=w
$$

(d) For $\mathrm{q} \in$ int $\mathbf{Q}$ we have $\mathrm{R}^{*}(\mathrm{q},-\mathrm{DE}(\mathrm{q}))=\mathcal{S}_{\mathrm{sl}}(\Psi(\mathrm{q}))=: \mathrm{S}(\mathrm{q})$;

(e) $\mathfrak{D}_{0}(\Psi(\mathrm{q}))=\int_{0}^{T}\{\mathrm{R}(\mathrm{q}, \dot{\mathrm{q}})+\mathrm{S}(\mathrm{q})\} \mathrm{d} t$.

In part (e) it is crucial to observe that for differentiable $t \mapsto \mathrm{q}(t)$ we cannot guarantee that $t \mapsto c(t)=\Psi(\mathrm{q}(t))$ is differentiable as well, since $\mathrm{q}(t)$ need not remain in the interior of $\mathbf{Q}$. However, for $\mathfrak{D}_{0}$ we only need continuity of $c$ and the differentiability of $t \mapsto Q_{\mathrm{fa}} c(t)=\mathrm{q}(t)$, where we used $\mathbf{q}=Q_{\mathrm{fa}} \Psi(\mathrm{q})$, see (3.9).

Proof. For part (a), we use that $\Psi$ is differentiable in int $\mathbf{Q}$. Differentiating the relation $Q_{\mathrm{fa}} \Psi(\mathrm{q})=\mathrm{q}$ yields $Q_{\mathrm{fa}} \mathrm{D} \Psi(\mathrm{q})=I_{m_{\mathrm{fa}}}$. In particular, this implies that $\mathrm{D} \Psi(\mathrm{q}) Q_{\mathrm{fa}}$ is a projection, and hence also its transpose $Q_{\mathrm{fa}}^{\top} \mathrm{D} \Psi(\mathrm{q})^{\top}$.

To show part (b) we first use the chain rule $\mathrm{DE}(\mathrm{q})=\mathrm{D} \Psi(\mathrm{q})^{\top} \mathrm{DE}(\Psi(\mathrm{q}))$. With lemma 3.7 and part (a) we have that $\mathrm{D} \mathcal{E}(\Psi(\mathrm{q}))=Q_{\mathrm{fa}}^{\top} \mathrm{D} \Psi(\mathrm{q})^{\top} \mathrm{D} \mathcal{E}(\Psi(\mathrm{q}))$, which yields

$$
\begin{aligned}
\partial_{\zeta} \mathrm{R}^{*}(\mathrm{q},-\mathrm{DE}(\mathrm{q})) & =Q_{\mathrm{fa}}^{\top} \mathrm{D}_{\xi} \mathcal{R}_{\mathrm{sl}}^{*}\left(\Psi(\mathrm{q}),-Q_{\mathrm{fa}}^{\top} \mathrm{D} \Psi(\mathrm{q})^{\top} \mathrm{D} \mathcal{E}(\Psi(\mathrm{q}))\right) \\
& =Q_{\mathrm{fa}}^{\top} \mathrm{D}_{\xi} \mathcal{R}_{\mathrm{sl}}^{*}(\Psi(\mathrm{q}),-\mathrm{D} \mathcal{E}(\Psi(\mathrm{q})))=Q_{\mathrm{fa}} \boldsymbol{R}_{\mathrm{sl}}(\Psi(\mathrm{q})) .
\end{aligned}
$$


For (c) we establish the relation $\mathrm{R}^{*}=\mathcal{L} \mathrm{R}$ via the Legendre transformation $\mathcal{L}$ :

$$
\begin{aligned}
(\mathcal{L R}(q, \cdot))(\zeta) & =\sup \left\{\zeta \cdot w-\mathbf{R}(\mathbf{q}, w) \mid w \in \mathbb{R}^{m_{\mathrm{fa}}}\right\} \\
& =\sup \left\{\zeta \cdot w+\sup \left\{-\mathcal{R}_{\mathrm{sl}}(\Psi(\mathbf{q}), v) \mid Q_{\mathrm{fa}} v=w\right\} \mid w \in \mathbb{R}^{m_{\mathrm{fa}}}\right\} \\
& =\sup \left\{\zeta \cdot w-\mathcal{R}_{\mathrm{sl}}(\Psi(\mathbf{q}), v) \mid Q_{\mathrm{fa}} v=w\right\} \\
& =\sup \left\{\zeta \cdot Q_{\mathrm{fa}} v-\mathcal{R}_{\mathrm{sl}}(\Psi(\mathbf{q}), v) \mid v \in \mathbb{R}^{i_{*}}\right\} \\
& =\mathcal{R}_{\mathrm{sl}}^{*}\left(\Psi(\mathbf{q}), Q_{\mathrm{fa}}^{\top} \zeta\right)=\mathbf{R}^{*}(\mathbf{q}, \zeta) .
\end{aligned}
$$

Part (d) follows similarly as part (b) by inserting $\mathrm{DE}(\mathrm{q})=\mathrm{D} \Psi(\mathrm{q})^{\top} \mathrm{DE}(\Psi(\mathrm{q})$ ) and $\mathrm{D} \mathcal{E}(\Psi(\mathrm{q}))=Q_{\mathrm{fa}}^{\top} \mathrm{D} \Psi(\mathrm{q})^{\top} \mathrm{D} \mathcal{E}(\Psi(\mathrm{q}))$ into the definition of $\mathrm{R}^{*}$ via $\mathcal{R}_{\mathrm{sl}}^{*}$.

For part (e) we first observe that $\mathcal{S}_{\mathrm{sl}}(\Psi(\mathrm{q}))=\mathrm{S}(\mathrm{q})$ for all $q \in \mathbf{Q}$ by definition. For the rate part $\mathcal{R}_{\text {eff }}(c, \dot{c})$ part (c) established that the dependence on $\dot{c}$ is only through $Q_{\mathrm{fa}} \dot{c}$. But relation (3.9) gives $\frac{\mathrm{d}}{\mathrm{d} t} Q_{\mathrm{fa}} \Psi(\mathrm{q}(t))=\dot{\mathrm{q}}(t)$, and the relation $\mathcal{R}_{\text {eff }}(c, \dot{c})=\mathcal{R}_{\text {eff }}(\Psi(\mathrm{q})$, D $\Psi(\mathrm{q}) \dot{\mathrm{q}})=\mathrm{R}(\mathrm{q}, \dot{\mathrm{q}})$ holds even $q(t)$ touching the boundary of $\mathbf{Q}$.

The next result shows that the reduced gradient-flow equation (4.3) indeed is the limiting equation for the fast-slow RRE (2.15) in the sense that for solutions $c^{\varepsilon}:[0, T] \rightarrow \mathbf{C}$ any accumulation point q : $[0, T] \rightarrow \mathbf{Q}$ of the family $\left(Q_{\mathrm{fa}} \varepsilon^{\varepsilon}\right)$ solves indeed (4.3). The assumptions on the initial conditions $c^{\varepsilon}(0)$ are special to avoid a potential jump at $t=0$, see section 2.4. The proof is based on the EDP and follows [Mie16, theorem 3.3.3] or [MMP21, lemma 2.8] with some special care because of the degeneracies and singularities of the limiting problem.

Proposition 4.3 (Reduced limiting equation). Consider a fast-slow DBRS $\left(A, B, c_{*}\right.$, $\left.\widehat{\kappa}^{\varepsilon}\right)$ satisfying the UFEC (3.5) and let $c^{\varepsilon}:[0, T] \rightarrow \mathbb{R}^{i_{*}}$ be a family of solutions of the fast-slow RRE (2.15). If along a subsequence (not relabeled) we have $c^{\varepsilon} \rightarrow c^{0}$ in $L^{1}([0, T] ; \mathbf{C})$ and $c^{\varepsilon}(0) \rightarrow \bar{c}_{0} \in \mathscr{M}_{\mathrm{sl}}$, then $Q_{\mathrm{fa}} \varepsilon^{\varepsilon} \rightarrow \mathrm{q}:=Q_{\mathrm{fa}} c^{0}$ weakly in $W^{1,1}([0, T] ; \mathbf{Q})$ and strongly in $C^{0}([0, T] ; \mathbf{Q})$, and $\mathrm{q}$ solves the reduced gradient-flow equation (4.3) with initial condition $\mathrm{q}(0)=Q_{\mathrm{fa}} \bar{c}_{0}$.

Proof. The solutions $c^{\varepsilon}$ satisfy the $\operatorname{EDB} \mathcal{E}\left(c^{\varepsilon}(T)\right)+\mathfrak{D}_{\varepsilon}\left(c^{\varepsilon}\right)=\mathcal{E}\left(c^{\varepsilon}(0)\right)$. Using $c^{\varepsilon} \rightarrow$ $c^{0}$ in $\mathrm{L}^{1}\left([0, T] ; \mathbb{R}^{i_{*}}\right)$ and $\limsup _{\varepsilon \rightarrow 0^{+}} \mathfrak{D}_{\varepsilon}\left(c^{\varepsilon}\right) \leqslant \lim _{\varepsilon \rightarrow 0^{+}} \mathcal{E}\left(c^{\varepsilon}(0)\right)=\mathcal{E}\left(\bar{c}_{0}\right)<\infty$, we obtain $\mathrm{q}^{\varepsilon}:=Q_{\mathrm{fa}} \varepsilon^{\varepsilon} \rightarrow \mathrm{q}$ weakly in $W^{1,1}([0, T] ; \mathbf{Q})$ and strongly in $C^{0}([0, T] ; \mathbf{Q})$ by invoking theorem 5.1(b). Moreover, because of $\bar{c}_{0} \in \mathscr{M}_{\mathrm{sl}}$ and $\mathrm{q}^{\varepsilon}(0)=Q_{\mathrm{fa}} \varepsilon^{\varepsilon}(0) \rightarrow Q_{\mathrm{fa}} \bar{c}_{0}$ we have $\mathrm{q}(0)=Q_{\mathrm{fa}} \bar{c}_{0}$ and hence $\bar{c}_{0}=\Psi(\mathrm{q}(0))$ and $\mathcal{E}\left(\bar{c}_{0}\right)=\mathrm{E}(\mathrm{q}(0))$. Passing to the limit $\varepsilon \rightarrow 0^{+}$using the liminf estimate in $\mathfrak{D}_{\varepsilon} \stackrel{\Gamma_{\mathrm{E}}}{\longrightarrow} \mathfrak{D}_{0}$ we arrive at

$$
\mathrm{E}(\mathrm{q}(T))+\mathfrak{D}_{0}(\Psi(\mathrm{q})) \leqslant \mathcal{E}\left(c^{0}(T)\right)+\mathfrak{D}_{0}\left(c^{0}\right) \leqslant \mathcal{E}\left(\bar{c}_{0}\right)=\mathrm{E}(\mathrm{q}(0)) .
$$

Because $\mathfrak{D}_{0}(\Psi(\cdot))$ has the $\mathrm{R} \oplus \mathrm{R}^{*}$ structure (cf proposition 4.2(d + e)) the EDP shows that $\mathrm{q}$ solves the reduced RRE (4.3).

\subsection{The projection approach}

By contrast to section 4.1 above, in this section we maintain the variable $c$. First, we justify the limiting equation (2.21) with the constraint $c \in \mathscr{M}_{\mathrm{sl}}$ and the Lagrange multiplier $\lambda(t) \in \Gamma_{\text {fa }}$. Secondly, we show that for positive solutions the evolution can be written as an ODE involving a suitable projection. Finally, we compare this to the reduced limiting equation (4.3).

Proposition 4.4 (Limiting equation with constraint). For a fast-slow DBRS (A, B, $c_{*}$, $\left.\widehat{\kappa}^{\varepsilon}\right)$ satisfying the UFEC (3.5) we consider a family $c^{\varepsilon}:[0, T] \rightarrow \mathbb{R}^{i_{*}}$ of solutions of the 
fast-slow RRE (2.15). If along a subsequence (not relabeled) we have $c^{\varepsilon} \rightarrow c^{0}$ in $L^{1}([0, T] ; \mathbf{C})$ and $c^{\varepsilon}(0) \rightarrow \bar{c}_{0} \in \mathscr{M}_{\mathrm{s}}$, then there exists $c \in C^{0}([0, T] ; \mathbf{C})$ such that $c(t)=c^{0}(t)$ a.e. in $[0, T]$, $c(0)=\bar{c}_{0}, Q_{\mathrm{fa}} c \in W^{1,1}([0, T] ; \mathbf{Q})$, and $c$ solves the limiting equation with constraint:

$$
\dot{c}(t)=\boldsymbol{R}_{\mathrm{sl}}(c(t))+\lambda(t), \quad \lambda(t) \in \Gamma_{\mathrm{fa}}, \quad c(t) \in \mathscr{M}_{\mathrm{sl}} .
$$

Proof. We proceed as in the proof of proposition 4.3 but stay with $c$ rather than reducing to $\mathrm{q}=Q_{\mathrm{fa}} c$. The solutions $c^{\varepsilon}$ satisfy the EDB $\mathcal{E}\left(c^{\varepsilon}(T)\right)+\mathfrak{D}_{\varepsilon}\left(c^{\varepsilon}\right)=\mathcal{E}\left(c^{\varepsilon}(0)\right)$. Using $c^{\varepsilon} \rightarrow c^{0}$ in $\mathrm{L}^{1}\left([0, T] ; \mathbb{R}^{i_{*}}\right)$ and $\lim \sup _{\varepsilon \rightarrow 0^{+}} \mathfrak{D}_{\varepsilon}\left(c^{\varepsilon}\right) \leqslant \lim _{\varepsilon \rightarrow 0} \mathcal{E}\left(c^{\varepsilon}(0)\right)=\mathcal{E}\left(\bar{c}_{0}\right)<\infty$, we have $Q_{\mathrm{fa}} c^{\varepsilon} \rightarrow$ $\mathrm{q}$ weakly in $W^{1,1}([0, T] ; \mathbf{C})$ and strongly in $C^{0}\left([0, T] ; \mathbb{R}^{i_{*}}\right)$, see theorem $5.1(\mathrm{~b})$. With this we define $c(t)=\Psi(\mathrm{q}(t))$ for $t \in[0, T]$ such that $c \in C^{0}([0, T] ; \mathbf{C})$ and $Q_{\mathrm{fa}} c(t)=\mathrm{q}(t)$.

Passing to the limit $\varepsilon \rightarrow 0^{+}$in the EDB we obtain $\mathcal{E}(c(T))+\mathfrak{D}_{0}(c) \leqslant \mathcal{E}(c(0))$, and the EDP gives the gradient-flow equation

$$
\dot{c} \in \partial_{\xi} \mathcal{R}_{\mathrm{eff}}^{*}(c,-\mathrm{D} \mathcal{E}(c))=\partial_{\xi}\left(\mathcal{R}_{\mathrm{sl}}^{*}(c,-\mathrm{D} \mathcal{E}(c))+\chi_{\Gamma_{\mathrm{fa}}^{\perp}}(-\mathrm{D} \mathcal{E}(c))\right) .
$$

For a linear subspace $Y \subset \mathbb{R}^{i_{*}}$ the set-valued convex subdifferential $\partial \chi_{Y^{\perp}}(\xi)$ equals $Y$ for $\xi \in$ $Y^{\perp}$ and $\emptyset$ otherwise, hence the last relation has the form

$$
\dot{c} \in \partial_{\xi} \mathcal{R}_{\mathrm{sl}}^{*}(c,-\mathrm{D} \mathcal{E}(c))+\Gamma_{\mathrm{fa}}=\boldsymbol{R}_{\mathrm{sl}}(c)+\Gamma_{\mathrm{fa}} \quad \text { and } \quad \mathrm{D} \mathcal{E}(c) \in \Gamma_{\mathrm{fa}}^{\perp} .
$$

With lemma 3.7 we can replace the last constraint by $c(t) \in \mathscr{M}_{\text {sl }}$, and (4.5) is established.

To obtain an ODE of the form $\dot{c}=V(c)$ instead of the limiting equation (4.5) with constraint, we have to resolve the constraint $\mathrm{D} \mathcal{E}(c) \in \Gamma_{\mathrm{fa}}^{\perp}$. For any curve $s \rightarrow \widetilde{c}(s) \in \mathscr{M}_{\mathrm{sl}} \cap \mathbf{C}_{+}$we have $\mathrm{D} \mathcal{E}(\widetilde{c}(s)) \in \Gamma_{\mathrm{fa}}^{\perp}$ and taking the derivative with respect to $s$, we find

$$
\dot{\widetilde{c}}(s) \in \mathrm{T}_{\widetilde{c}(s)} \mathscr{M}_{\mathrm{sl}} \quad \text { and } \quad \mathrm{D}^{2} \mathcal{E}(\widetilde{c}(s)) \dot{\widetilde{c}}(s) \in \Gamma_{\mathrm{fa}}^{\perp} .
$$

Hence, for $c \in \mathscr{M}_{\mathrm{sl}} \cap \mathbf{C}_{+}$the tangent space $\mathrm{T}_{c} \mathscr{M}_{\mathrm{sl}}$ of $\mathscr{M}_{\mathrm{sl}}$ at $c$ is given by

$$
\mathrm{T}_{c} \mathscr{M}_{\mathrm{sl}}=(\mathbb{H}(c))^{-1} \Gamma_{\text {fa }}^{\perp} \quad \text { with } \quad \mathbb{H}(c):=\mathrm{D}^{2} \mathcal{E}(c)=\operatorname{diag}\left(1 / c_{1}, \ldots, 1 / c_{i_{*}}\right) .
$$

With this we obtain the following representation of the limiting equation, which matches that in [Bot03, theorem 2(b)] and [DLZ18, theorem 4.5]. Our result is more general, since we do not need to assume that the stoichiometric vectors $\left\{\gamma^{r} \mid r \in R_{\mathrm{fa}}\right\}$ are linearly independent.

Proposition 4.5 (Limiting equation for $\mathbf{c} \in \mathbf{C}_{+}$). A curve $c:[0, T] \rightarrow \mathbf{C}_{+}$is a solution of (4.5) if and only if

$$
\dot{c}=(I-\mathbb{P}(c)) \boldsymbol{R}_{\mathrm{sl}}(c) \text { and } c(0) \in \mathscr{M}_{\mathrm{sl}} .
$$

where the projector $\mathbb{P}(c) \in \mathbb{R}^{i_{*} \times i_{*}}$ is defined via $\operatorname{im} \mathbb{P}(c)=\Gamma_{\mathrm{fa}}$ and $\operatorname{ker} \mathbb{P}(c)=\mathbb{H}(c)^{-1} \Gamma_{\mathrm{fa}}^{\perp}$.

Proof. Step 1. Definition of the projector $\mathbb{P}(c)$ : the projector is uniquely defined if $Y_{\mathrm{R}}:=\Gamma_{\mathrm{fa}}$ and $Y_{\mathrm{K}}:=\mathbb{H}(c)^{-1} \Gamma_{\text {fa }}^{\perp}$ provide a direct decomposition of $\mathbb{R}^{i_{*}}$. Assuming $v \in Y_{\mathrm{R}} \cap Y_{\mathrm{K}}$ we have $v \in \Gamma_{\mathrm{fa}}$ and $\mathbb{H}(c) v \in \Gamma_{\mathrm{fa}}^{\perp}$. This implies $v \cdot \mathbb{H}(c) v=0$, but since $\mathbb{H}(c)$ is positive definite we arrive at $v=0$. Hence, $Y_{\mathrm{R}} \cap Y_{\mathrm{K}}=\{0\}$. Obviously, $\operatorname{dim} Y_{\mathrm{R}}+\operatorname{dim} Y_{\mathrm{K}}=i_{*}$, so that $\mathbb{R}^{i_{*}}=Y_{\mathrm{R}} \oplus$ $Y_{\mathrm{K}}$ is established.

Step 2. (4.7) $\Longrightarrow(4.5)$ : we set $\lambda(t)=-\mathbb{P}(c(t)) \boldsymbol{R}_{\mathrm{sl}}(c)$, and with (4.7) we obtain

$$
\dot{c}(t)=\boldsymbol{R}_{\mathrm{sl}}(c(t))-\mathbb{P}(c(t)) \boldsymbol{R}_{\mathrm{sl}}(c(t))=\boldsymbol{R}_{\mathrm{sl}}(c(t))+\lambda(t) \quad \text { with } \lambda(t) \in \Gamma_{\mathrm{fa}} .
$$


Moreover, $\mathbb{P}(c(t)) \dot{c}(t)=\mathbb{P}(c)(I-\mathbb{P}(c)) \boldsymbol{R}_{\mathrm{sl}}(c)=0$, which implies $\dot{c} \in \mathbb{H}(c)^{-1} \Gamma_{\mathrm{fa}}^{\perp}=\mathrm{T}_{c(t)} \mathscr{M}_{\mathrm{sl}}$. Hence, with $c(0) \in \mathscr{M}_{\mathrm{sl}}$ we obtain $c(t) \in \mathscr{M}_{\mathrm{sl}}$ for all $t \in[0, T]$, and (4.5) is established.

Step 3. (4.5) $\Longrightarrow$ (4.7): from $c(t) \in \mathscr{M}_{\mathrm{sl}}$ we obtain $\dot{c}(t) \in \mathrm{T}_{c(t)} \mathscr{M}_{\mathrm{sl}}=\mathbb{H}(c(t))^{-1} \Gamma_{\text {fa }}^{\perp}$ and conclude $0=\mathbb{P}(c) \dot{c}=\mathbb{P}(c) \boldsymbol{R}_{\mathrm{sl}}(c)+\mathbb{P}(c) \lambda$. Using $\lambda \in \operatorname{im} \mathbb{P}(c)=\Gamma_{\mathrm{fa}}$ we have $\mathbb{P}(c) \lambda=\lambda$ and find

$$
(I-\mathbb{P}(c)) \boldsymbol{R}_{\mathrm{sl}}(c)=\boldsymbol{R}_{\mathrm{sl}}(c)-\mathbb{P}(c) \boldsymbol{R}_{\mathrm{sl}}(c)=\boldsymbol{R}_{\mathrm{sl}}(c)+\mathbb{P}(c) \lambda=\boldsymbol{R}_{\mathrm{sl}}(c)+\lambda=\dot{c},
$$

which is the desired equation (4.7).

To compare the last result with the reduced limiting equation (4.3), we simply use the relation $c(t)=\Psi(\mathrm{q}(t))$ and the fact that $\Psi$ is smooth on int $\mathbf{Q}$. From this we obtain

$$
\begin{aligned}
(I-\mathbb{P}(c)) \boldsymbol{R}_{\mathrm{sl}}(c) & =\dot{c}=\mathrm{D} \Psi(\mathrm{q}) \dot{\mathrm{q}}=\mathrm{D} \Psi(\mathrm{q}(t)) Q_{\mathrm{fa}} \boldsymbol{R}_{\mathrm{sl}}(\Psi(\mathrm{q})) \\
& =\mathrm{D} \Psi(\mathrm{q}(t)) Q_{\mathrm{fa}} \boldsymbol{R}_{\mathrm{sl}}(c) .
\end{aligned}
$$

Thus, we can conclude that for $c=\Psi(\mathrm{q}) \in \mathscr{M}_{\mathrm{sl}}$ we have the identity

$$
(I-\mathbb{P}(c))=\mathrm{D} \Psi(\mathrm{q}) Q_{\mathrm{fa}},
$$

since the above identity must hold for all possible right-hand sides $\boldsymbol{R}_{\mathrm{sl}}$. This can also be shown by using the identity $c=\Psi\left(Q_{\mathrm{fa}} c\right)$ for all $c \in \mathscr{M}_{\mathrm{sl}}$ and taking derivatives in the direction $v \in \Gamma_{\mathrm{fa}}$ and $w \in \mathrm{T}_{c} \mathscr{M}_{\mathrm{sl}}$, respectively. In particular, this provides the explicit form of the projection of proposition 4.2(a).

\subsection{An example for the effective gradient system}

In the following example we consider a system with $i_{*}=5$ species and $r_{*}=2$ bimolecular reactions, one fast and one slow. As a result we obtain a limiting equation with one reaction that is no longer of mass-action type but involves all species. Taking a further EDP limit (done only formally) we recover a trimolecular reaction of mass-action type again.

We consider the following two reactions

$$
\text { fast: } X_{1}+X_{2} \leftrightharpoons X_{3} \text { and slow: } X_{3}+X_{4} \rightleftharpoons X_{5} \text {, }
$$

which give rise to the two stoichiometric vectors

$$
\gamma^{\mathrm{fa}}=(1,1,-1,0,0)^{\top} \quad \text { and } \quad \gamma^{\mathrm{sl}}=(0,0,1,1,-1)^{\top} .
$$

Assuming the steady state $c_{*}=(1,1, \varrho, 1,1)^{\top}$ and the reaction coefficients $\widehat{\kappa}^{\varepsilon}=\left(\kappa^{\mathrm{fa}} / \varepsilon, \kappa^{\mathrm{sl}}\right)$ the RRE (2.15) takes the form

$$
\dot{c}=-\frac{\kappa^{\mathrm{fa}} \varrho^{1 / 2}}{\varepsilon}\left(c_{1} c_{2}-c_{3} / \varrho\right) \gamma^{\mathrm{fa}}-\kappa^{\mathrm{sl}} \varrho^{1 / 2}\left(c_{3} c_{4} / \varrho-c_{5}\right) \gamma^{\mathrm{sl}} .
$$

The slow manifold is $\mathscr{M}_{\mathrm{sl}}=\left\{c \in[0, \infty]^{5} \mid c_{1} c_{2}=c_{3} / \varrho\right\}$ and $\Gamma_{\mathrm{fa}}=\operatorname{span} \gamma^{\mathrm{fa}}$. With

$$
Q_{\mathrm{fa}}=\left(\begin{array}{ccccc}
1 & 0 & 1 & 0 & 0 \\
0 & 1 & 1 & 0 & 0 \\
0 & 0 & 0 & 1 & 0 \\
0 & 0 & 0 & 0 & 1
\end{array}\right)
$$


we obtain $\mathbf{Q}=\operatorname{im} Q_{\mathrm{fa}}=\left[0, \infty\left[{ }^{4}\right.\right.$. For $\mathbf{q} \in \mathbf{Q}$ it is easy to compute $\Psi_{\varrho}(\mathbf{q})$ as a minimizer of $c \mapsto \mathcal{E}(c)$ under the constraint $Q_{\mathrm{fa}} c=\mathrm{q}=\left(q_{1}, \ldots, q_{4}\right)$. We obtain

$$
\begin{aligned}
& \Psi_{\varrho}(\mathrm{q})=\left(q_{1}-a_{\varrho}\left(q_{1}, q_{2}\right), q_{2}-a_{\varrho}\left(q_{1}, q_{2}\right), a_{\varrho}\left(q_{1}, q_{2}\right), q_{3}, q_{4}\right)^{\top} \in \mathbf{C}=\left[0, \infty\left[^{5}\right.\right. \\
& \text { with } a_{\varrho}\left(q_{1}, q_{2}\right)=\frac{1}{2 \varrho}\left(1+\varrho q_{1}+\varrho q_{2}-\sqrt{\left(1+\varrho q_{1}+\varrho q_{2}\right)^{2}-4 \varrho^{2} q_{1} q_{2}}\right) \\
& \quad \in\left[0, \min \left\{q_{1}, q_{2}\right\}\right] .
\end{aligned}
$$

In particular, the UFEC (3.5) holds. Moreover, the positivity and monotonicity condition (3.6) can be checked easily with $\overline{\mathbf{q}}=(1,1,1,1)^{\top}$. We see that $c(\theta):=\Psi_{\varrho}(\mathbf{q}+\theta \overline{\mathrm{q}})$ for $\left.\left.\theta \in\right] 0,1\right]$ is given by

$$
\begin{aligned}
c(\theta)= & \left(q_{1}+\theta-a_{\varrho}\left(q_{1}+\theta, q_{2}+\theta\right), q_{2}\right. \\
& \left.+\theta-a_{\varrho}\left(q_{1}+\theta, q_{2}+\theta\right), a_{\varrho}\left(q_{1}+\theta, q_{2}+\theta\right), q_{3}+\theta, q_{4}+\theta\right)^{\top} .
\end{aligned}
$$

Clearly we have $c(\theta)_{i}>0$, since $c(\theta)_{i}=0$ would imply $q_{i}+\theta=0$. Differentiating with respect to $\theta$, we obtain

$$
c^{\prime}(\theta)=\left(1-a_{\varrho}^{\prime}[\theta], 1-a_{\varrho}^{\prime}[\theta], a_{\varrho}^{\prime}[\theta], 1,1\right)^{\top} \quad \text { with } a_{\varrho}^{\prime}[\theta]=\frac{\varrho\left(c_{1}(\theta)+c_{2}(\theta)\right)}{1+\varrho\left(c_{1}(\theta)+c_{2}(\theta)\right)},
$$

which implies that $c^{\prime}(\theta)_{i}>0$. Hence, $\Psi_{\varrho}(\mathbf{q}+\theta \bar{q})_{i}=c(\theta)_{i} \geqslant c(0)_{i}=\Psi(\mathrm{q})$, i.e. the monotonicity condition (3.6) holds.

We investigate the reduced system. First, we observe that the reduced limiting equation (4.3) is given by

$$
\begin{aligned}
\dot{\mathrm{q}} & =Q_{\mathrm{fa}} \boldsymbol{R}_{\mathrm{sl}}\left(\Psi_{\varrho}(\mathrm{q})\right)=-\kappa^{\mathrm{sl}} \varrho^{1 / 2}\left(\frac{a_{\varrho}\left(q_{1}, q_{2}\right) q_{3}}{\varrho}-q_{4}\right) \widehat{\gamma} \\
\text { with } \widehat{\gamma} & :=Q_{\mathrm{fa}} \gamma^{\mathrm{sl}}=(1,1,1,-1)^{\top} .
\end{aligned}
$$

Since $a_{\varrho}$ is not a monomial, this RRE is no longer of mass-action type.

According to section 4.1 the gradient structure $\left(\mathbf{Q}, \mathrm{E}_{\varrho}, \mathbf{R}_{\varrho}\right)$ for $(4.8)$ is given via

$$
\begin{aligned}
\mathrm{E}_{\varrho}(\mathrm{q})= & \mathcal{E}\left(\Psi_{\varrho}(\mathrm{q})\right)=\lambda_{\mathrm{B}}\left(q_{1}-a\right)+\lambda_{\mathrm{B}}\left(q_{2}-a\right) \\
& +\varrho \lambda_{\mathrm{B}}(a / \varrho)+\lambda_{\mathrm{B}}\left(q_{3}\right)+\left.\lambda_{\mathrm{B}}\left(q_{4}\right)\right|_{a=a_{\varrho}\left(q_{1}, q_{2}\right)}, \\
\mathrm{R}_{\varrho}^{*}(\mathrm{q}, \zeta)= & \mathcal{R}_{\mathrm{sl}}\left(\Psi_{\varrho}(\mathrm{q}), Q_{\mathrm{fa}}^{\top} \zeta\right)=\kappa^{\mathrm{sl}}\left(a_{\varrho}\left(q_{1}, q_{2}\right) q_{3} q_{4}\right)^{1 / 2} \mathrm{C}^{*}\left(\zeta_{1}+\zeta_{2}+\zeta_{3}-\zeta_{4}\right) .
\end{aligned}
$$

The energy $\mathrm{E}_{\varrho}$ is no longer of Boltzmann type, because the previously uncoupled densities $c_{1}$, $c_{2}$, and $c_{3}$ are now constrained to lie on $\mathscr{M}_{\text {sl }}$, i.e. $c_{1} c_{2}=c_{3}$. Nevertheless, the form is close to a mass-action type for the trimolecular reaction $Y_{1}+Y_{2}+Y_{3} \rightleftharpoons Y_{4}$.

To recover an exact trimolecular reaction of mass-action type, one has to perform another limit, namely $\varrho \rightarrow 0^{+}$, which means that the species $X_{3}$ is no longer observed, but still exists on a microscopic reaction pathway. For the limit $\varrho \rightarrow 0^{+}$we simply observe the expansion

$$
a_{\varrho}\left(q_{1}, q_{2}\right)=\varrho q_{1} q_{2}+O\left(\varrho^{2}\right) \text { for } \varrho \rightarrow 0^{+},
$$


which implies $\Psi_{\varrho}(q) \rightarrow \Psi_{0}(q):=\left(q_{1}, q_{2}, 0, q_{3}, q_{4}\right)^{\mathrm{T}}$. If we additionally choose $\kappa^{\mathrm{sl}}=\bar{\kappa} / \varrho^{1 / 2}$ and insert the expansion for $a_{\varrho}$ we obtain

$$
\begin{aligned}
\mathrm{E}_{\varrho}(\mathbf{q}) \rightarrow \mathrm{E}_{0}(\mathbf{q}) & =\sum_{j=1}^{4} \lambda_{\mathrm{B}}\left(q_{j}\right), \\
\mathbf{R}_{\varrho}^{*}(\mathbf{q}, \zeta) \rightarrow \mathrm{R}_{0}^{*}(q, \zeta) & =\bar{\kappa}\left(q_{1} q_{2} q_{3} q_{4}\right)^{1 / 2} \mathrm{C}^{*}\left(\zeta_{1}+\zeta_{2}+\zeta_{3}-\zeta_{4}\right) .
\end{aligned}
$$

Clearly, this is the GS generating the RRE of the trimolecular reaction $X_{1}+X_{2}+X_{4} \rightleftharpoons X_{5}$. Of course, it is possible to show that this convergence is again an EDP-convergence with tilting of the $\operatorname{GSs}\left(\mathbf{Q}, \mathrm{E}_{\varrho}, \mathbf{R}_{\varrho}\right)$ to the effective system $\left(\mathbf{Q}, \mathbf{E}_{0}, \mathbf{R}_{0}\right)$.

\section{Proof of theorem 3.4}

Here we will show the $\Gamma$-convergence of the dissipation functionals, namely $\mathfrak{D}_{\varepsilon} \stackrel{\mathrm{M}_{\mathrm{E}}}{\longrightarrow} \mathfrak{D}_{0}$. As usual the proof consists in three parts: (i) compactness of the sequences $\left(c^{\varepsilon}\right)$ satisfying $\mathfrak{D}_{\mathcal{\varepsilon}}\left(c^{\varepsilon}\right) \leqslant$ $C$, (ii) the liminf estimate, and the (iii) the limsup estimate, which needs the construction of recovery sequences.

All the following results are derived under the assumptions of theorem 3.4: the fast-slow DBRS $\left(A, B, c_{*}, \widehat{\kappa}^{E}\right)$ satisfies the UFEC (3.5). For constructing the recovery sequence in section 5.3, we need additionally the positivity and monotonicity assumption (3.6) for $\Psi$.

\subsection{Compactness}

In the definition of $\mathfrak{D}_{\varepsilon} \stackrel{\mathrm{M}_{\mathrm{E}}}{\longrightarrow} \mathfrak{D}_{0}$ we consider sequences $c^{\varepsilon} \rightarrow c^{0}$ in $\mathrm{L}^{1}([0, T] ; \mathbf{C})$ that additionally satisfy $\sup _{\varepsilon \in[0,1[, t \in[0, T]} \mathcal{E}\left(c^{\varepsilon}(t)\right) \leqslant C$. The aim is to extract a strongly converging subsequence $c^{\varepsilon} \rightarrow c^{0}$, such that we can talk about pointwise convergence almost everywhere. This will be necessary in the liminf estimate because we cannot rely on convexity, in contrast to the linear theory developed in [MiS19]. The compactness is derived via two quite different arguments that complement each other and reflect the underlying fast-slow structure, which is seen on the local level via the decomposition of $\mathrm{T}_{c} \mathbf{C}=\mathbb{R}^{i_{*}}$ in the direct sum of $\Gamma_{\mathrm{fa}}$ and $\mathrm{T}_{c} \mathscr{M}_{\mathrm{sl}}$, see step 1 in the proof of proposition 4.5. First, we derive time regularity for the slow part of the reactions. Secondly, we prove convergence towards the slow manifold which then provides the remaining information for the whole sequence.

Theorem 5.1 (Compactness via dissipation bound). Consider a family $\left(c^{\varepsilon}\right)_{\varepsilon>0}$ with $c^{\varepsilon} \rightarrow c^{0}$ in $L^{1}([0, T] ; \mathbf{C}), \sup _{\varepsilon>0, t \in[0, T]} \mathcal{E}\left(c^{\varepsilon}(t)\right) \leqslant M_{\text {ener }}<\infty$, and $\mathfrak{D}_{\varepsilon}\left(c^{\varepsilon}\right) \leqslant M_{\text {diss }}<\infty$. Then, we have

(a) $c^{\varepsilon}(\cdot)$ is bounded in $L^{\infty}([0, T]$; $\boldsymbol{C})$;

(b) $Q_{\mathrm{fa}} c^{\varepsilon} \rightarrow Q_{\mathrm{fa}} c^{0}$ weakly in $W^{1,1}\left([0, T] ; \mathbb{R}^{m_{\mathrm{fa}}}\right)$ and strongly in $C^{0}\left([0, T] ; \mathbb{R}^{m_{\mathrm{fa}}}\right)$;

(c) $c^{0}(t)=\widetilde{c}(t):=\Psi\left(Q_{\mathrm{fa}} c^{0}(t)\right) \in \mathscr{M}_{\text {sl }}$ for a.a. $t \in[0, T]$, and, in particular $\widetilde{c} \in C^{0}([0, T]$, C);

(d) $c^{\varepsilon} \rightarrow c^{0}$ in $L^{p}([0, T] ; C)$ strongly for all $p \in[1, \infty[$.

We emphasize that $c^{0}$ and $\widetilde{c}$ may be different, and this happens even for solutions, if near $t=0$ a jump develops such that (cf section 2.4)

$$
\lim c^{\varepsilon}(0)=: c_{0} \neq \bar{c}_{0}:=\lim _{\tau \rightarrow 0^{+}}\left(\lim _{\varepsilon \rightarrow 0^{+}} c^{\varepsilon}(\tau)\right) .
$$


Before giving the detailed proof we provide two preliminary results that underpin the two complementary arguments of the proof.

For deriving bounds on the time derivatives, one heuristically sees that for fixed $(c, \xi)$ we have $\mathcal{R}_{\varepsilon}^{*}(c, \xi) \nearrow \mathcal{R}_{\text {eff }}^{*}(c, \xi)$ as $\varepsilon \rightarrow 0$. By duality, this implies $\mathcal{R}_{\varepsilon}(c, v) \searrow \mathcal{R}_{\text {eff }}(c, v)$. This already shows that control of time derivatives has to be obtained from $\mathcal{R}_{\text {eff }}(c, \cdot)$, which only controls $Q_{\mathrm{fa}} \dot{c}$ because $\mathcal{R}_{\text {eff }}(c, v)=\mathcal{R}_{\text {eff }}(c, w)$ if $Q_{\mathrm{fa}} v=Q_{\mathrm{fa}} w$, see (3.4b).

Proposition 5.2 (Effective dissipation potential). For all $\varepsilon>0$ we have $\mathcal{R}_{\varepsilon}(c, v) \geqslant$ $\mathcal{R}_{\text {eff }}(c, v)$ for all $(c, v) \in \mathbf{C} \times \mathbb{R}^{i_{*}}$. Moreover, $\mathcal{R}_{\text {eff }}$ takes the form

$$
\begin{aligned}
\mathcal{R}_{\text {eff }}(c, v) & =\widetilde{\mathcal{R}}\left(c, Q_{\mathrm{fa}} v\right) \\
\text { where } \widetilde{\mathcal{R}}(c, \mathrm{q}) & :=\sup \left\{\zeta \cdot \mathrm{q}-\mathcal{R}_{\mathrm{sl}}^{*}\left(c, Q_{\mathrm{fa}}^{\top} \zeta\right) \mid \zeta \in \mathbb{R}^{m_{\mathrm{fa}}}\right\} .
\end{aligned}
$$

Proof. We first use the standard relation from linear algebra: $\operatorname{im}\left(Q_{\mathrm{fa}}^{\top}\right)=\left(\operatorname{ker}\left(Q_{\mathrm{fa}}\right)\right)^{\perp}=\Gamma_{\mathrm{fa}}^{\perp}$. By construction of $\Gamma_{\mathrm{fa}}$ we have $\mathcal{R}_{\mathrm{fa}}^{*}(c, \xi)=0$ for $\xi \in \Gamma_{\mathrm{fa}}^{\perp}$ and obtain

$$
\begin{aligned}
\mathcal{R}_{\varepsilon}^{*}(c, \xi) & =\mathcal{R}_{\mathrm{sl}}^{*}(c, \xi)+\frac{1}{\varepsilon} \mathcal{R}_{\mathrm{fa}}^{*}(c, \xi) \leqslant \mathcal{R}_{\mathrm{eff}}^{*}(c, \xi):=\mathcal{R}_{\mathrm{sl}}^{*}(c, \xi)+\chi_{\Gamma_{\mathrm{fa}}^{\top}}(\xi) \\
& =\mathcal{R}_{\mathrm{sl}}^{*}(c, \xi)+\chi_{\mathrm{im} Q_{\mathrm{fa}}^{\top}(\xi) .}
\end{aligned}
$$

Applying the Legendre-Fenchel transformation we obtain

$$
\begin{aligned}
\mathcal{R}_{\varepsilon}(c, v) & \geqslant \mathcal{R}_{\text {eff }}(c, v)=\sup \left\{v \cdot \xi-\mathcal{R}_{\mathrm{sl}}^{*}(c, \xi) \mid \xi \in \operatorname{im}\left(Q_{\mathrm{fa}}^{\top}\right)\right\} \\
& =\sup \left\{v \cdot Q_{\mathrm{fa}}^{\top} \zeta-\mathcal{R}_{\mathrm{sl}}^{*}\left(c, Q_{\mathrm{fa}}^{\top} \zeta\right) \mid \zeta \in \mathbb{R}^{m_{\mathrm{fa}}}\right\}=\widetilde{\mathcal{R}}\left(c, Q_{\mathrm{fa}} v\right),
\end{aligned}
$$

which provides the desired estimate as well as the representation via $\widetilde{\mathcal{R}}$.

The second result concerns the convergence of points towards the slow manifold $\mathscr{M}_{\text {sl }}$, and the crucial property here is the UFEC (3.5) that guarantees the relation

$$
\left\{\Psi(\mathrm{q}) \mid \mathbf{q} \in \mathbf{Q} \subset \mathbb{R}^{m_{\mathrm{fa}}}\right\}=: \mathscr{M}_{\mathrm{sl}} \stackrel{! !}{=} \mathscr{E}_{\mathrm{fa}}^{\text {Lemma }} \stackrel{2.4}{=}\left\{c \in \mathbf{C} \mid \mathcal{S}_{\mathrm{fa}}(c)=0\right\} .
$$

Lemma 5.3 (Convergence towards $\mathscr{M}_{\mathrm{sl}}$ ). For bounded sequences $\left(c^{n}\right)_{n \in \mathbb{N}}$ in $\mathbf{C}$ we have

$$
Q_{\mathrm{fa}} c^{n} \rightarrow \mathrm{q} \quad \text { and } \quad \mathcal{S}_{\mathrm{fa}}\left(c^{n}\right) \rightarrow 0 \quad \Longrightarrow \quad c^{n} \rightarrow \Psi(\mathrm{q}) .
$$

Proof. Without loss of generality we may assume $c^{n} \rightarrow \bar{c}$. Hence we have $Q_{\mathrm{fa}} c^{n} \rightarrow Q_{\mathrm{fa}} \bar{c}=\mathrm{q}$. Moreover, the continuity of $\mathcal{S}_{\text {fa }}$ gives $0=\lim \mathcal{S}_{\mathrm{fa}}\left(c^{n}\right)=\mathcal{S}_{\mathrm{fa}}(\bar{c})$. Thus, we have $\bar{c} \in \mathscr{E}_{\mathrm{fa}} \cap \mathbf{C}_{\mathrm{q}}^{\mathrm{fa}}$. Now, the UFEC (see (3.11)) gives $\bar{c}=\Psi(\mathrm{q})$ which is the desired result.

We are now ready to establish the main compactness result.

Proof of theorem 5.1. Part (i): from the energy bound $\mathcal{E}\left(c^{\varepsilon}(t)\right) \leqslant M_{\text {ener }}<\infty$ and the coercivity of $\mathcal{E}$ we obtain an $\mathrm{L}^{\infty}$ bound for $c^{\varepsilon}$, namely $0 \leqslant c_{j}^{\varepsilon}(t) \leqslant\left|c^{\varepsilon}(t)\right| \leqslant\left\|c^{\varepsilon}\right\|_{\mathrm{L}^{\infty}} \leqslant M_{\text {ener }}$.

Part (ii): to provide a lower bound on $\mathcal{R}_{\text {eff }}$ we first observe an upper bound on $\mathcal{R}_{\mathrm{sl}}^{*}$, namely

$$
\begin{aligned}
\mathcal{R}_{\mathrm{sl}}^{*}\left(c^{\varepsilon}, Q_{\mathrm{fa}}^{\top} \zeta\right) & \leqslant \sum_{r \in R_{\mathrm{sl}}} \kappa_{r} M_{\mathrm{ener}}^{\left(\alpha^{r}+\beta^{r}\right) / 2} \mathrm{C}^{*}\left(\gamma^{r} \cdot Q_{\mathrm{fa}}^{\top} \zeta\right) \leqslant b_{M} \mathrm{C}^{*}\left(b_{Q}|\zeta|\right) \\
\text { with } b_{Q} & =\max _{r \in R_{\mathrm{sl}}}\left|Q_{\mathrm{fa}} \gamma^{r}\right|
\end{aligned}
$$


where we used $0 \leqslant c_{j}^{\varepsilon} \leqslant M_{\text {ener }}$ from part (i). Using the Legendre-Fenchel transformation and proposition 5.2 we obtain the lower bound

$$
\begin{aligned}
\mathcal{R}_{\varepsilon}\left(c^{\varepsilon}, v\right) & \geqslant \widetilde{\mathcal{R}}\left(c^{\varepsilon}, Q_{\mathrm{fa}} v\right) \geqslant \sup \left\{Q_{\mathrm{fa}} v \cdot \zeta-b_{M} \mathrm{C}^{*}\left(b_{Q}|\zeta|\right) \mid \zeta \in \mathbb{R}^{m_{\mathrm{fa}}}\right\} \\
& =b_{M} \mathrm{C}\left(\frac{\left|Q_{\mathrm{fa}} v\right|}{b_{M} b_{Q}}\right) .
\end{aligned}
$$

Using the bound $M_{\text {diss }}$ for the dissipation functionals, the family satisfies

$$
\int_{0}^{T} \mathrm{C}\left(\frac{\left|Q_{\mathrm{fa}} \dot{c}^{\varepsilon}(t)\right|}{b_{M} b_{Q}}\right) \mathrm{d} t \leqslant \int_{0}^{T} \frac{1}{b_{M}} \mathcal{R}_{\varepsilon}\left(c^{\varepsilon}(t), \dot{c}^{\varepsilon}(t)\right) \mathrm{d} t \leqslant \frac{\mathfrak{D}_{\varepsilon}\left(c^{\varepsilon}\right)}{b_{M}} \leqslant \frac{M_{\mathrm{diss}}}{b_{M}} .
$$

Since $\mathrm{C}(s) \geqslant \frac{1}{2}|s| \log (1+|s|)$ for all $s \in \mathbb{R}(\operatorname{cf}[\operatorname{MiS} 19$, equation (A.2)]) we have a uniform superlinear bound for $Q_{\mathrm{fa}} \dot{c}^{\varepsilon}$. Thus, there exists a subsequence (not relabeled) such that $Q_{\mathrm{fa}} \dot{c}^{\varepsilon} \rightarrow$ $\mathrm{w}$ in $\mathrm{L}^{1}\left([0, T] ; \mathbb{R}^{m_{\mathrm{fa}}}\right)$. Moreover, $Q_{\mathrm{fa}} c^{\varepsilon}$ is equicontinuous (cf [MiS19, proposition 5.9]), which implies $Q_{\mathrm{fa}} c^{\varepsilon} \rightarrow \mathrm{q}^{0}$ in $C^{0}([0, T] ; \mathbf{Q})$.

Because of $c^{\varepsilon} \rightarrow c^{0}$ we conclude $\mathbf{q}^{0}=Q_{\mathrm{fa}} c^{0} \in W^{1,1}([0, T] ; \mathbf{Q})$ and $\dot{\mathrm{q}}=\mathrm{w}$. Since the limit is unique, we also know that the whole family converges.

Part (iii): the dissipation bound gives the estimate $\int_{0}^{T} \mathcal{S}_{\mathrm{fa}}\left(c^{\varepsilon}(t)\right) \mathrm{d} t \leqslant \varepsilon M_{\mathrm{diss}}$. Using $\mathcal{S}_{\mathrm{fa}}(c) \geqslant 0$ this implies that $f_{\varepsilon}=\mathcal{S}_{\mathrm{fa}} \circ c^{\varepsilon}$ converges to 0 in $\mathrm{L}^{1}([0, T])$. Thus, we may choose a subsequence (not relabeled) such that $f_{\varepsilon}(t) \rightarrow 0$ a.e. in $[0, T]$.

By the continuity $\mathcal{S}_{\mathrm{fa}}$ and $\left|c^{\varepsilon}(t)\right| \leqslant M_{\text {ener }}$ we also know that $\left(f_{\varepsilon}(t)\right)_{\varepsilon \in[0,1[}$ is bounded, while part (ii) provides the convergence $Q_{\mathrm{fa}} c^{\varepsilon}(t) \rightarrow \mathrm{q}^{0}(t)=Q_{\mathrm{fa}} c^{0}(t)$. Hence, lemma 5.3 guarantees $c^{\varepsilon}(t) \rightarrow \widetilde{c}(t):=\Psi\left(Q_{\mathrm{fa}} c^{0}(t)\right)$ a.e. in $[0, T]$. By $c^{\varepsilon} \rightarrow c^{0}$ we have $c^{0}(t)=\widetilde{c}(t)$ a.e.

Since $\Psi$ is continuous by proposition 3.6, also $\widetilde{c}=\Psi\left(Q_{\mathrm{fa}} c^{0}\right)$ is continuous.

Part (iv): this follows via part (i), the pointwise a.e. convergence established in the proof of part (iii), and from the dominated-convergence theorem.

\subsection{Liminf estimate}

The liminf estimate follows in a straightforward manner by using the fact that the velocity part $\mathcal{R}_{\varepsilon}$ in $\mathfrak{D}_{\varepsilon}$ satisfies the monotonicity $\mathcal{R}_{\varepsilon} \geqslant \mathcal{R}_{\text {eff }}$, see proposition 5.2, and that the slope part $\mathcal{S}_{\varepsilon}$ takes the simple form $\mathcal{S}_{\mathrm{sl}}+\frac{1}{\varepsilon} \mathcal{S}_{\text {fa }}$.

Theorem 5.4 (Liminf estimate). Let $\left(c^{\varepsilon}\right)_{\varepsilon>0}$ with $c^{\varepsilon} \rightarrow c^{0}$ in $L^{1}([0, T]$; C) as in theorem 5.1. Then we have the estimate $\mathfrak{D}_{0}\left(c^{0}\right) \leqslant \liminf _{\varepsilon \rightarrow 0^{+}} \mathfrak{D}_{\varepsilon}\left(c^{\varepsilon}\right)$.

Proof. We may assume that $\alpha_{*}:=\liminf _{\varepsilon \rightarrow 0} \mathfrak{D}_{\varepsilon}\left(c^{\varepsilon}\right)<\infty$, since otherwise the desired estimate is trivially satisfied. This implies $\mathcal{S}_{\mathrm{fa}}\left(c^{0}(t)\right)=0$ a.e. in $[0, T]$ as in the previous proof. We define the functional

$$
\Im(c, \mathrm{q}):=\int_{0}^{T} \mathcal{F}(c(t), \mathrm{q}(t)) \mathrm{d} t \quad \text { with } \mathcal{F}(c, \mathrm{w})=\widetilde{\mathcal{R}}(c, \mathrm{w})+\mathcal{S}_{\mathrm{sl}}(c) .
$$

Then, using $\mathcal{R}_{\varepsilon} \geqslant \mathcal{R}_{\text {eff }}$ and $\mathcal{S}_{\varepsilon} \geqslant \mathcal{S}_{\text {sl }}$, we have

$$
\mathfrak{D}_{\varepsilon}\left(c^{\varepsilon}\right) \geqslant \mathfrak{I}\left(c^{\varepsilon}, Q_{\mathrm{fa}} \dot{c}^{\varepsilon}\right) \quad \text { and } \quad \mathfrak{D}_{0}\left(c^{0}\right)=\mathfrak{I}\left(c^{0}, Q_{\mathrm{fa}} \dot{c}^{0}\right),
$$

where the last identity follows from the construction of the density $\mathcal{F}$ via $\widetilde{\mathcal{R}}$ and $\mathcal{S}_{\text {sl }}$, and $\mathcal{S}_{0}(c(t))=\mathcal{S}_{\mathrm{sl}}(c(t))$ a.e. because of $\mathcal{S}_{\mathrm{fa}}\left(c^{0}(t)\right)=0$.

Thus, it suffices to show the lower semicontinuity $\Im\left(c^{0}, Q_{\mathrm{fa}} \dot{c}^{0}\right) \leqslant \liminf _{\varepsilon \rightarrow 0} \Im\left(c^{\varepsilon}, Q_{\mathrm{fa}} \dot{c}^{\varepsilon}\right)$. Using the strong convergence $c^{\varepsilon} \rightarrow c^{0}$ in $\mathrm{L}^{p}([0, T] ; \mathbf{C})$ and the weak convergence $Q_{\mathrm{fa}} \dot{c}^{\varepsilon} \rightarrow$ 
$Q_{\mathrm{fa}} \dot{c}^{0}$ in $\mathrm{L}^{1}\left([0, T] ; \mathbb{R}^{m_{\mathrm{fa}}}\right)$, see theorem 5.1(b $\left.+\mathrm{d}\right)$, this follows by Ioffe's theorem (cf [FoL07, theorem 7.5] if $\mathcal{F}: \mathbf{C} \times \mathbb{R}^{m_{\mathrm{fa}}} \rightarrow[0, \infty]$ is lower semicontinuous. However, the lower semicontinuity of $(c, \mathrm{w}) \mapsto \mathcal{F}(c, \mathrm{w})=\widetilde{\mathcal{R}}(c, \mathrm{w})+\mathcal{S}_{\text {sl }}(c)$ follows immediately from the continuity of $\mathcal{S}_{\text {sl }}$ and the by Legendre transforming the continuous function $(c, \zeta) \mapsto \mathcal{R}_{\mathrm{sl}}^{*}\left(c, Q_{\mathrm{fa}}^{\top} \zeta\right)$.

This finishes the proof of theorem 5.4.

\subsection{Construction of the recovery sequence}

In this section we construct the recovery sequence which completes the proof of the Mosco convergence $\mathfrak{D}_{\varepsilon} \stackrel{\mathrm{M}_{\mathrm{E}}}{\longrightarrow} \mathfrak{D}_{0}$ with energy constraint. Below in step 1, we will need the positivity and monotonicity condition (3.6) for $\theta \mapsto \Psi(\mathbf{q}+\theta \overline{\mathbf{q}})$.

Theorem 5.5 (Limsup estimate). Let $c^{0} \in L^{1}\left([0, T]\right.$; C) with $\sup _{t \in[0, T]} \mathcal{E}\left(c^{0}(t)\right)<\infty$. Then there exists a family $\left(c^{\varepsilon}\right)_{\varepsilon \in] 0,1]}$ with $\sup _{t \in[0, T], \varepsilon \in] 0,1]} \mathcal{E}\left(c^{\varepsilon}(t)\right) \leqslant M_{\text {ener }}<\infty, c^{\varepsilon} \rightarrow c^{0}$ strongly in $L^{1}([0, T] ; \mathbf{C})$, and $\lim _{\varepsilon \rightarrow 0} \mathfrak{D}_{\varepsilon}\left(c^{\varepsilon}\right)=\mathfrak{D}_{0}\left(c^{0}\right)$.

Proof. We prove the theorem in several steps. In steps 1 and 2 we show that it is sufficient to consider $c^{0} \in W^{1, \infty}([0, T] ; \mathbf{C})$ with $c_{j}^{0}(t) \geqslant \underline{c}>0$, where we only work in $\mathfrak{D}_{0}$ which has the advantage that $\mathcal{R}_{\text {eff }}(c, \dot{c})$ only depends on (q, $\left.\dot{\mathrm{q}}\right)=\left(Q_{\mathrm{fa}} c, Q_{\mathrm{fa}} \dot{c}\right)$, see section 4.1. In step 3 we construct a recovery sequence, and in step 4 we conclude with a diagonal argument.

Step 0: to start with we may assume $\mathfrak{D}_{0}\left(c^{0}\right)<\infty$. Indeed, if $\mathfrak{D}_{0}\left(c^{0}\right)=\infty$, then we choose $c^{\varepsilon}=c^{0}$ and theorem 5.4 gives $\lim \inf _{\varepsilon \rightarrow 0} \mathfrak{D}_{\varepsilon}\left(c^{\varepsilon}\right) \geqslant \mathfrak{D}_{0}\left(c^{0}\right)=\infty$, which means $\mathfrak{D}_{\varepsilon}\left(c^{\varepsilon}\right) \rightarrow \infty$ as desired.

Step 1. Reducing to positive curves $c^{0}$ : for $c^{0}$ with $\mathfrak{D}_{0}\left(c^{0}\right)<\infty$ we know that $Q_{\mathrm{fa}} c^{0} \in$ $W^{1,1}([0, T] ; \mathbf{Q})$ and $c^{0} \in C^{0}([0, T] ; \mathbf{C})$ after choosing the continuous representative $c^{0}=\widetilde{c}$, see theorem 5.1. Exploiting the positivity and monotonicity condition (3.6) we now set

$$
\left.\underline{c}^{l}(t):=\Psi\left(\mathbf{q}(t)+\theta_{l} \overline{\mathbf{q}}\right) \quad \text { with } \theta_{l}=\frac{1}{l+1} \in\right] 0,1[\text { for all } t \in[0, T] .
$$

By this condition, we know that $\underline{c}^{l}(t)$ lies in $\mathbf{C}_{+}$for all $t \in[0, T]$, such that the continuity of $\underline{c}^{l}$ guarantees that for each $l$ there exists a $\delta_{l}>0$ such that $\underline{c}_{i}^{l}(t) \geqslant \delta_{l}$ for all $i \in I$ and $t \in[0, T]$.

By the continuity of $\Psi$ we have $\underline{c}^{l} \rightarrow c^{0}$ uniformly and hence strongly in $\mathrm{L}^{1}([0, T] ; \mathbf{C})$. We now show

$$
\mathfrak{D}_{0}\left(\underline{c}^{l}\right)=\int_{0}^{T}\left\{\mathcal{R}_{\text {eff }}\left(\underline{c}^{l}(t), \underline{\dot{c}}^{l}(t)\right)+\mathcal{S}_{0}\left(\underline{c}^{l}(t)\right)\right\} \mathrm{d} t \rightarrow \mathfrak{D}_{0}\left(c^{0}\right) \quad \text { as } \quad l \rightarrow 0 .
$$

For the second part, we use $\underline{c}^{l}(t) \in \mathscr{M}_{\text {sl }}$ by construction via $\Psi$, and the continuity of $\mathcal{S}_{\text {sl }}$ yields $\mathcal{S}_{0}\left(\underline{c}^{l}(t)\right)=\mathcal{S}_{\mathrm{sl}}\left(\underline{c}^{l}(t)\right) \rightarrow \mathcal{S}_{\mathrm{sl}}\left(c^{0}(t)\right)=\mathcal{S}_{0}\left(c^{0}(t)\right)$ uniformly in $[0, T]$.

For the first part we use (i) the special form of $\mathcal{R}_{\text {eff }}$ derived in proposition 5.2, namely $\mathcal{R}_{\text {eff }}(c, v)=\widetilde{\mathcal{R}}\left(c, Q_{\mathrm{fa}} v\right)$, where $\widetilde{\mathcal{R}}(c, \cdot)$ is the Legendre transform of $\mathcal{R}_{\mathrm{sl}}^{*}\left(c, Q^{\top} \cdot\right)$. Moreover, the cosh-type dual dissipation potential $\mathcal{R}_{\mathrm{sl}}^{*}$ as defined in (2.7b) or (2.15) enjoys (ii) a monotonicity property namely $\mathcal{R}_{\mathrm{sl}}^{*}(c, \xi) \leqslant \mathcal{R}_{\mathrm{sl}}^{*}(\widetilde{c}, \xi)$ or equivalently $\mathcal{R}_{\mathrm{sl}}(c, v) \geqslant \mathcal{R}_{\mathrm{sl}}(\widetilde{c}, v)$ if $c \leqslant \widetilde{c}$ componentwise. This can be exploited because of the monotonicity condition (3.6) using $\underline{c}^{l}(t) \geqslant c^{0}(t)$ 
componentwise. With $Q_{\mathrm{fa}} \underline{\dot{c}}^{l}(t)=\dot{\mathrm{q}}(t)$ for all $l \in \mathbb{N}$ we obtain

$$
\begin{aligned}
\int_{0}^{T} \mathcal{R}_{\text {eff }}\left(\underline{c}^{l}, \underline{\dot{c}}^{l}(t)\right) \mathrm{d} t & \stackrel{(\mathrm{i})}{=} \int_{0}^{T} \widetilde{\mathcal{R}}\left(\underline{c}^{l}, \dot{\mathrm{q}}(t)\right) \mathrm{d} t \\
& \stackrel{(\mathrm{ii})}{\longrightarrow} \int_{0}^{T} \widetilde{\mathcal{R}}\left(c^{0}, \dot{\mathrm{q}}(t)\right) \mathrm{d} t \stackrel{(\mathrm{i})}{=} \int_{0}^{T} \mathcal{R}_{\text {eff }}\left(c^{0}, \dot{c}^{0}\right) \mathrm{d} t,
\end{aligned}
$$

where the convergence $\stackrel{\text { (ii) }}{\longrightarrow}$ follows from the dominated-convergence theorem, since the integrands on the left-hand side are bounded by that on the right-hand side and we have pointwise convergence. With this we have established the desired convergence (5.2).

Step 2. Reducing to bounded derivative $\dot{\mathrm{q}}=Q_{\mathrm{fa}} \dot{c}$ : because of step 1, we can now assume

$$
c^{0}(t) \in \mathbf{C}_{\delta}:=\left\{c \in \mathbf{C}|| c \mid \leqslant 1 / \delta, c_{i} \geqslant \delta \quad \text { for all } i \in I\right\} \quad \text { for all } t \in[0, T]
$$

where $\delta>0$. Moreover, as in [MiS19, step 2(b) of proof of theorem 5.12] we find $\Lambda^{*}$ such that

$$
c, \widetilde{c} \in \mathbf{C}_{\delta} \quad \text { and } \quad|c-\widetilde{c}| \leqslant \alpha<\frac{1}{2 \Lambda^{*}} \Longrightarrow \widetilde{\mathcal{R}}(\widetilde{c}, \mathbf{w}) \leqslant\left(1+\Lambda^{*} \alpha\right) \widetilde{\mathcal{R}}(c, \mathrm{w}) .
$$

With this we can estimate $\mathcal{R}_{\mathrm{sl}}^{*}(c, \cdot)$ from below and hence $\mathcal{R}_{\text {eff }}$ from above. Moreover, we can use the Lipschitz continuity of $c \mapsto \mathcal{R}_{\varepsilon}^{*}$.

For $\mathbf{q}(t)=Q_{\mathrm{fa}} c^{0} \in W^{1,1}([0, T] ; \mathbf{Q})$ we define the piecewise affine interpolants $\widehat{\mathrm{q}}^{k}$ via

$$
\begin{gathered}
\widehat{\mathrm{q}}^{k}\left((n+\theta) 2^{-k} T\right)=(1-\theta) \mathbf{q}\left(n 2^{-k} T\right)+\theta \mathbf{q}\left((n+1) 2^{-k} T\right) \\
\text { for } \theta \in[0,1], n \in\left\{0, \ldots, 2^{k}-1\right\}
\end{gathered}
$$

and the piecewise constant interpolant $\overline{\mathrm{q}}^{k}\left((n+\theta) 2^{-k} T\right)=\mathrm{q}\left(2^{-k} n T\right)$ for $\theta \in[0,1$ [. We also set $\widehat{c}^{k}(t)=\Psi\left(\widehat{\mathrm{q}}^{k}(t)\right)$ and $\bar{c}^{k}(t)=\Psi\left(\overline{\mathrm{q}}^{k}(t)\right)$. By standard arguments we have

$$
\left\|\bar{c}^{k}-\widehat{c}^{k}\right\|_{L^{\infty}}+\left\|\widehat{c}^{k}-c^{0}\right\|_{L^{\infty}}=: \alpha_{k} \rightarrow 0 \quad \text { for } k \rightarrow \infty .
$$

As in step 1 we again find $\int_{0}^{T} \mathcal{S}_{0}\left(\widehat{c}^{k}(t)\right) \mathrm{d} t \rightarrow \int_{0}^{T} \mathcal{S}_{0}\left(c^{0}(t)\right) \mathrm{d} t$. To treat the velocity part we use both interpolants to obtain the estimate

$$
\begin{aligned}
\int_{0}^{T} \mathcal{R}_{\text {eff }}\left(\widehat{c}^{k}, \dot{\vec{c}}^{k}\right) \mathrm{d} t & =\int_{0}^{T} \widetilde{\mathcal{R}}\left(\widehat{c}^{k}, \dot{\hat{\mathrm{q}}}^{k}\right) \mathrm{d} t \leqslant\left(1+\Lambda^{*} \alpha_{k}\right) \int_{0}^{T} \widetilde{\mathcal{R}}\left(\bar{c}^{k}, \dot{\widehat{\mathrm{q}}}^{k}\right) \mathrm{d} t \\
& \stackrel{(\mathrm{J})}{\leqslant}\left(1+\Lambda^{*} \alpha_{k}\right) \int_{0}^{T} \widetilde{\mathcal{R}}\left(\bar{c}^{k}, \dot{\mathrm{q}}\right) \mathrm{d} t \leqslant\left(1+\Lambda^{*} \alpha_{k}\right)^{2} \int_{0}^{T} \widetilde{\mathcal{R}}\left(c^{0}, \dot{\mathrm{q}}\right) \mathrm{d} t \\
& =\left(1+\Lambda^{*} \alpha_{k}\right)^{2} \int_{0}^{T} \mathcal{R}_{\text {eff }}\left(c^{0}, \dot{c}^{0}\right) \mathrm{d} t,
\end{aligned}
$$

(J)

where $\stackrel{(J)}{\leqslant}$ indicates the use of Jensen's inequality applied to the convex integrand $\widetilde{\mathcal{R}}\left(\bar{c}^{k}(t), \cdot\right)$, which is independent of $t$ in the intervals $] 2^{-k} n T, 2^{-k}(n+1) T$ [. Combining this with the slope part and using $\alpha_{k} \rightarrow 0$ we obtain the desired estimate $\lim \sup _{k \rightarrow \infty} \mathfrak{D}_{0}\left(\widehat{c}^{k}\right) \leqslant \mathfrak{D}_{0}\left(c^{0}\right)$, which is of course a limit because of the liminf estimate in theorem 5.4.

Step 3. The limsup for $\varepsilon \rightarrow 0^{+}$: by steps 1 and 2 it is sufficient to consider $c^{0} \in$ $W^{1, \infty}([0, T] ; \mathbf{C})$ with $c^{0}(t)=\Psi(\mathbf{q}(t)) \in \mathbf{C}_{\delta}$ for some $\delta>0$. For these functions we can now 
use the constant recovery sequence $c^{\varepsilon}=c^{0}$, i.e. we will show

$$
\mathfrak{D}_{\varepsilon}\left(c^{0}\right)=\int_{0}^{T}\left\{\mathcal{R}_{\varepsilon}\left(c^{0}, \dot{c}^{0}\right)+\mathcal{S}_{\varepsilon}\left(c^{0}\right)\right\} \mathrm{d} t \rightarrow \mathfrak{D}_{0}\left(c^{0}\right)=\int_{0}^{T}\left\{\mathcal{R}_{\text {eff }}\left(c^{0}, \dot{c}^{0}\right)+\mathcal{S}_{0}\left(c^{0}\right)\right\} \mathrm{d} t
$$

for $\varepsilon \rightarrow 0^{+}$. Because of $c^{0}(t) \in \mathscr{M}_{\mathrm{sl}}$ we have $\mathcal{S}_{\varepsilon}\left(c^{0}(t)\right)=\mathcal{S}_{\mathrm{sl}}\left(c^{0}(t)\right)=\mathcal{S}_{0}\left(c^{0}(t)\right)$, so the second summand of the integral $\mathfrak{D}_{\varepsilon}\left(c^{0}\right)$ converges trivially.

Recall that $\Gamma=\operatorname{span}\left\{\gamma^{r} \mid r \in R=R_{\mathrm{sl}} \dot{\cup} R_{\mathrm{fa}}\right\}$ and define a projection $\mathbb{Q}$ on $\mathbb{R}^{i_{*}}$ with $\operatorname{im} \mathbb{Q}=\Gamma$ giving $\operatorname{ker} \mathbb{Q}^{\top}=\Gamma^{\perp}$. With this we can estimate the dual dissipation potential $\mathcal{R}_{\varepsilon}^{*}$ from below:

$$
\mathcal{R}_{\varepsilon}^{*}(c, \xi) \geqslant \mathcal{R}_{1}^{*}(c, \xi) \geqslant b_{*}\left|\mathbb{Q}^{\top} \xi\right|^{2} .
$$

To see this use $\mathrm{C}^{*}(\sigma) \geqslant \frac{1}{2} \sigma^{2}$ and $\left(c^{\alpha^{r}} c^{\beta^{r}}\right)^{1 / 2} \geqslant \delta^{\left(\alpha^{r}+\beta^{r}\right) / 2}$ for all $r \in R$.

By Legendre-Fenchel transformation we obtain an upper bound for $\mathcal{R}_{\varepsilon}$, where we use $\dot{c}^{0} \in$ $\Gamma$, i.e. $\mathbb{Q} \dot{c}^{0}(t)=\dot{c}^{0}(t)(\mathrm{cf}$ lemma 2.5$)$ :

$$
\mathcal{R}_{\varepsilon}\left(c^{0}(t), \dot{c}^{0}(t)\right) \leqslant \mathcal{R}_{1}\left(c^{0}(t), \dot{c}^{0}(t)\right) \leqslant \frac{1}{4 b_{*}}\left|\mathbb{Q} \dot{c}^{0}(t)\right|^{2}=\frac{1}{4 b_{*}}\left|\dot{c}^{0}(t)\right|^{2}
$$

From $c^{0} \in W^{1, \infty}([0, T] ; \mathbf{C})$ we see that $t \mapsto \mathcal{R}_{1}\left(c^{0}(t), \dot{c}^{0}(t)\right)$ lies in $\mathrm{L}^{\infty}([0, T])$ and thus provides an integrable majorant for $t \mapsto \mathcal{R}_{\varepsilon}\left(c^{0}(t), \dot{c}^{0}(t)\right)$. However, the convergence $\mathcal{R}_{\varepsilon}^{*}(c, \xi) \nearrow$ $\mathcal{R}_{0}^{\varepsilon}=\mathcal{R}_{\mathrm{sl}}^{*}+\chi_{\Gamma_{\mathrm{fa}}^{\perp}}$ for $\varepsilon \rightarrow 0^{+}$implies $\mathcal{R}_{\varepsilon}(c, v) \searrow \mathcal{R}_{\mathrm{eff}}(c, v)$ for all $(c, v) \in \mathbf{C}_{\delta} \times \mathbb{R}^{i_{*}}$. Hence, Lebesgue's dominated convergence theorem gives

$$
\int_{0}^{T} \mathcal{R}_{\varepsilon}\left(c^{0}(t), \dot{c}^{0}(t)\right) \mathrm{d} t \rightarrow \int_{0}^{T} \mathcal{R}_{\mathrm{eff}}\left(c^{0}(t), \dot{c}^{0}(t)\right) \mathrm{d} t \quad \text { for } \varepsilon \rightarrow 0^{+},
$$

and (5.3) is established.

Step 4. Diagonal sequence: the full recovery sequence for a general $c^{0}$ with $\mathfrak{D}_{0}\left(c^{0}\right)<\infty$ is obtained via $\mathbf{q}(t)=Q_{\mathrm{fa}} c^{0}(t)$ as a diagonal sequence $c^{\varepsilon}=\Psi\left(\widehat{\mathbf{q}}^{k(\varepsilon)}(t)+\theta_{l(\varepsilon)} \overline{\mathbf{q}}\right)$, where the functions $k(\varepsilon)$ and $l(\varepsilon)$ are suitably chosen such that $c^{\varepsilon} \rightarrow c^{0}$ strongly in $\mathrm{L}^{1}([0, T] ; \mathbf{C})$ and $\mathfrak{D}_{\varepsilon}\left(c^{\varepsilon}\right) \rightarrow \mathfrak{D}_{0}\left(c^{0}\right)$. It is also clear from the construction that $\left\|c^{\varepsilon}\right\|_{L^{\infty}} \leqslant 1+\left\|c^{0}\right\|_{L^{\infty}}$ such that the uniform energy bound $\mathcal{E}\left(c^{\varepsilon}(t)\right) \leqslant M_{\text {ener }}$ holds.

\section{Acknowledgments}

The research of AM and AS was partially supported by Deutsche Forschungsgemeinschaft (DFG) through the Collaborative Research Center SFB 1114 'Scaling Cascades in Complex Systems' (Project No. 235221301), Subproject C05 'Effective models for materials and interfaces with multiple scales'. The research of MAP was partially supported by NWO grant 613.001.552, project 'Large Deviations and Gradient Flows'. The authors are grateful to Michiel Renger for helpful and stimulating discussions.

\section{ORCID iDs}

Alexander Mielke (D) https://orcid.org/0000-0002-4583-3888

Mark A Peletier (D) https://orcid.org/0000-0001-9663-3694 


\section{References}

[AGS05] Ambrosio L, Gigli N and Savaré G 2005 Gradient Flows in Metric Spaces and in the Space of Probability Measures (Lectures in Mathematics ETH Zürich) (Basel: Birkhäuser)

[Att84] Attouch H 1984 Variational Convergence of Functions and Operators (Boston, MA: Pitman)

[Bot03] Bothe D 2003 Instantaneous limits of reversible chemical reactions in presence of macroscopic convection J. Differ. Equ. 193 27-48

[Bra02] Braides A $2002 \Gamma$-Convergence for Beginners (Oxford: Oxford University Press)

[Bra14] Braides A 2014 Local Minimization, Variational Evolution and Gamma-Convergence (Lecture Notes in Mathematics vol 2094) (Berlin: Springer)

[Da193] Dal Maso G 1993 An Introduction to Г-Convergence (Boston, MA: Birkhäuser)

[DLZ18] Disser K, Liero M and Zinsl J 2018 Evolutionary $\Gamma$-convergence of gradient systems modeling slow and fast chemical reactions Nonlinearity 31 3689-706

[DFM19] Dondl P, Frenzel T and Mielke A 2019 A gradient system with a wiggly energy and relaxed EDP-convergence ESAIM Control Optim. Calc. Var. 2545

[FoL07] Fonseca I and Leoni G 2007 Modern Methods in the Calculus of Variations: $L^{p}$ Spaces (Berlin: Springer)

[FrL19] Frenzel T and Liero M 2019 Effective diffusion in thin structures via generalized gradient systems and EDP-convergence WIAS Preprint 2601

[Grm10] Grmela M 2010 Multiscale equilibrium and nonequilibrium thermodynamics in chemical engineering $A d v$. Chem. Eng. 39 75-129

[KaK13] Kang H-W and Kurtz T G 2013 Separation of time-scales and model reduction for stochastic reaction networks Ann. Appl. Probab. 23 529-83

[LM*17] Liero M, Mielke A, Peletier M A and Renger D R M 2017 On microscopic origins of generalized gradient structures Discrete Continuous Dyn. Syst. Ser. S 10 1-35

[MaM20] Maas J and Mielke A 2020 Modeling of chemical reaction systems with detailed balance using gradient structures J. Stat. Phys. 181 2257-303

[Mar15] Marcelin M R 1915 Contribution a l'étude de la cinétique physico-chimique Ann. Phys. 9 $120-231$

[Mie11] Mielke A 2011 A gradient structure for reaction-diffusion systems and for energy-driftdiffusion systems Nonlinearity 24 1329-46

[Mie17] Mielke A 2017 Uniform exponential decay for reaction-diffusion systems with complexbalanced mass-action kinetics Pattern of Dynamics (Springer Proceedings in Mathematics and Statistics vol 205) ed P Gurevich, J Hell, B Sandstede and A Scheel (Berlin: Springer) pp 149-71

[MHM15] Mielke A, Haskovec J and Markowich P A 2015 On uniform decay of the entropy for reactiondiffusion systems J. Dyn. Differ. Equ. 27 897-928

[MMP21] Mielke A, Montefusco A and Peletier M A 2021 Exploring families of energy-dissipation landscapes via tilting: three types of EDP convergence Contin. Mech. Thermodyn. 33 611-37

[Mie16] Mielke A 2012 On evolutionary $\Gamma$-convergence for gradient systems (chapter 3) Macroscopic and Large Scale Phenomena: Coarse Graining, Mean Field Limits and Ergodicity (Lecture Notes in Applied Mathematics and Mechanics vol 3) ed A Muntean, J Rademacher and A Zagaris (Berlin: Springer) pp 187-249

[MP*17] Mielke A, Patterson R I A, Peletier M A and Michiel Renger D R 2017 Non-equilibrium thermodynamical principles for chemical reactions with mass-action kinetics SIAM J. Appl. Math. 77 1562-85

[MPR14] Mielke A, Peletier M A and Renger D R M 2014 On the relation between gradient flows and the large-deviation principle, with applications to Markov chains and diffusion Potential Anal. 41 1293-327

[MiS19] Mielke A and Stephan A 2020 Coarse graining via EDP-convergence for linear fast-slow reaction systems Math. Models Methods Appl. Sci. 30 1765-807

[ÖtG97] Öttinger H C and Grmela M 1997 Dynamics and thermodynamics of complex fluids. II. Illustrations of a general formalism Phys. Rev. E 56 6633-55 
[Ste19] Stephan A 2019 On EDP-convergence for gradient systems with different time scales Proc. Appl. Math. Mech. 19 e201900366

[WiS17] Winkelmann S and Schütte C 2017 Hybrid models for chemical reaction networks: multiscale theory and application to gene regulatory systems J. Chem. Phys. 147114115

[Yon08] Yong W-A 2008 An interesting class of partial differential equations J. Math. Phys. 49033503 